\title{
Molybdenum-Catalyzed Ring-Closing Metathesis of Allenynes
}

\author{
Masahiro Murakami, * Sho Kadowaki, and Takanori Matsuda \\ Department of Synthetic Chemistry and Biological Chemistry, Kyoto University, \\ Katsura, Kyoto 615-8510, Japan
}

\section{Supporting Information}

\begin{tabular}{|c|c|}
\hline Table of Contents & S1 \\
\hline General Considerations & $\mathrm{S} 2$ \\
\hline Preparation and Characterization of Substrates & $\mathrm{S} 2$ \\
\hline $\begin{array}{l}\text { Experimental Procedure for RCM and } \\
\text { Characterization Data for Products }\end{array}$ & S8 \\
\hline${ }^{1} \mathrm{H}$ and ${ }^{13} \mathrm{C}$ NMR Spectra for Substrates & S14 \\
\hline${ }^{1} \mathrm{H}$ and ${ }^{13} \mathrm{C}$ NMR Spectra for Products & S46 \\
\hline
\end{tabular}


General. All manipulations were carried out in a nitrogen-filled gloved box and with standard Schlenk techniques under an argon atmosphere. Column chromatography was performed with silica gel $60 \mathrm{~N}$ (Kanto). Preparative thin-layer chromatography was performed with silica gel $60 \mathrm{PF}_{254}$ (Merck). NMR spectra were recorded on a Varian Gemini $2000\left({ }^{1} \mathrm{H}\right.$ at $300.77 \mathrm{MHz}$ and ${ }^{13} \mathrm{C} \mathrm{NMR}$ at $\left.75.46 \mathrm{MHz}\right)$ or a Varian Mercury $400\left({ }^{1} \mathrm{H}\right.$ at $400.44 \mathrm{MHz}$ ). All NMR data were obtained in $\mathrm{CDCl}_{3}$. Proton chemical shifts were referenced to the residual proton signal of the solvent at $7.26 \mathrm{ppm}$. Carbon chemical shifts were referenced to the carbon signal of the solvent at $77.00 \mathrm{ppm}$. High resolution mass spectra were recorded on a JEOL JMS-SX102A. IR spectra was recorded on a Shimadzu FTIR-8100 spectrometer.

Materials. 2,6-Diisopropylphenylimidoneophilidenemolybdenum(VI) bis(hexafluoro- $t$ butoxide) (Schrock catalyst, 1) was purchased from Strem. Toluene was distilled from sodium-benzophenone ketyl before using for catalytic reactions. All other commercially available chemical resources were used without further purifications.

\section{Typical Procedure for the Preparation of Tosylamine-bridged Allenynes ${ }^{1,2}$}

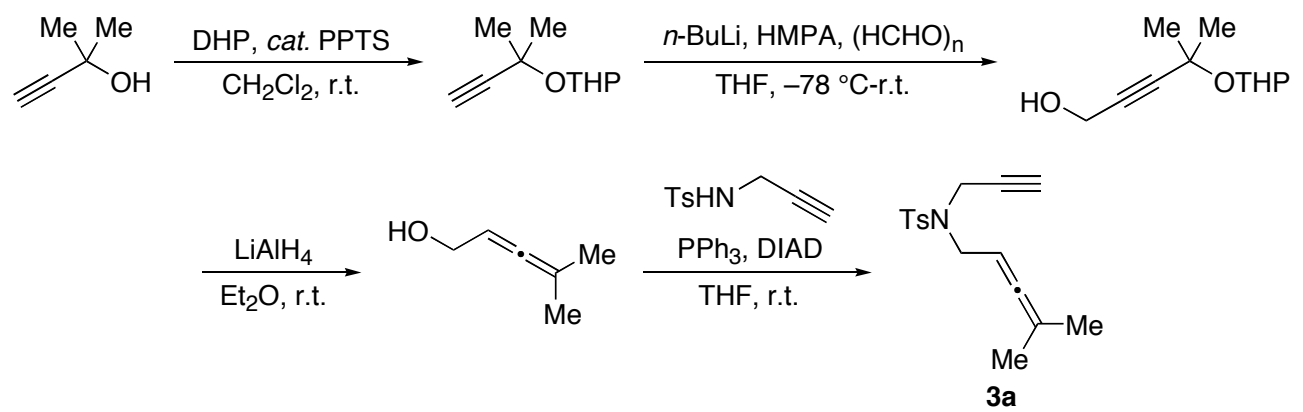

To a solution of pyridinium $p$-toluenesulfonate (PPTS) $(1.78 \mathrm{~g}, 7.10 \mathrm{mmol}, 5 \mathrm{~mol} \%)$ in $\mathrm{CH}_{2} \mathrm{Cl}_{2}(60 \mathrm{~mL})$ were added 2-methylbut-3-yne-2-ol $(12.0 \mathrm{~g}, 143 \mathrm{mmol})$ and 3,4dihydropyran (DHP) (50.0 g, $594 \mathrm{mmol})$. After being stirred at room temperature for $16 \mathrm{~h}$, the volatile materials were removed under reduced pressure. The residue was taken up in ether, washed with water, dried over $\mathrm{MgSO}_{4}$, and concentrated. Distillation under reduced pressure $\left(35^{\circ} \mathrm{C} / 1.8 \mathrm{mmHg}\right)$ gave pure 1,1-dimethylprop-2-ynyl tetrahydropyran-2-yl ether

(1) Cowie, J. S.; Landor, P. D.; Landor, S. R. J. Chem. Soc., Perkin Trans. 1 1973, 3807.

(2) Wender, P. A.; Glorius, F.; Husfeld, C. O.; Langkopf, E.; Love, J. A. J. Am. Chem. Soc. 1999, 121, 5348. 
(22.0 g, 90\%).

To a solution of 1,1-dimethylprop-2-ynyl tetrahydropyran-2-yl ether (4.00 g, 23.8 $\mathrm{mmol})$ in THF $(70.0 \mathrm{~mL})$ at $-78{ }^{\circ} \mathrm{C}$ was slowly added $n$-butyllithium (1.59 $\mathrm{M}$ hexane solution; $20 \mathrm{~mL})$ over $30 \mathrm{~min}$. HMPA $(5.0 \mathrm{~mL})$ was added to the mixture at $0{ }^{\circ} \mathrm{C}$, and then paraformaldehyde $(1.43 \mathrm{~g}, 47.6 \mathrm{mmol})$ was added. After being stirred at room temperature for $2 \mathrm{~h}$, the reaction was quenched by a phosphate buffer solution ( $\mathrm{pH}$ 6.9), and the mixture was extracted with ether. The organic layer was washed with water, dried over $\mathrm{MgSO}_{4}$, and concentrated. Distillation under reduced pressure $\left(100{ }^{\circ} \mathrm{C} / 1.8 \mathrm{mmHg}\right)$ gave pure 4-methyl4-(tetrahydropyran-2-yloxy)pent-2-yn-1-ol (3.40 g, 72\%).

To an ether suspension $(50 \mathrm{~mL})$ of lithium aluminum hydride $(1.57 \mathrm{~g}, 41.5 \mathrm{mmol})$ was added dropwise ether solution $(15 \mathrm{~mL})$ of 4-methyl-4-(tetrahydropyran-2-yloxy)pent-2-yn1-ol (3.29 g, $16.6 \mathrm{mmol}$ ) over $30 \mathrm{~min}$. After being stirred at room temperature for $3 \mathrm{~h}$, the reaction was quenched by addition of a mixture of Celite ${ }^{\circledR}$ and sodium sulfate decahydrate $(\mathrm{v} / \mathrm{v}=1 / 1)$, and the mixture was filtrated. The solvent was removed under reduced pressure, and the residue was distilled under reduced pressure $\left(35^{\circ} \mathrm{C} / 1.8 \mathrm{mmHg}\right)$ to give pure 4methylpenta-2,3-dien-1-ol (1.03 g, 63\%).

To a stirred solution of $N$-(prop-2-ynyl)tosylamine (584 $\mathrm{mg}, 2.79 \mathrm{mmol}$ ) in THF (7.0 $\mathrm{mL})$ were added successively triphenylphosphine $(1.39 \mathrm{~g}, 5.30 \mathrm{mmol})$, 4-methylpenta-2,3dien-1-ol (903 mg, $9.20 \mathrm{mmol}$ ), and diisopropylcarbodiimide (DIAD) (903 mg, $4.46 \mathrm{mmol}$ ) at $0{ }^{\circ} \mathrm{C}$. The reaction was warmed to room temperature over $30 \mathrm{~min}$ and was stirred at room temperature for $4 \mathrm{~h}$. The solvent was removed under reduced pressure, and the residue was purified by column chromatography on silica gel (hexane:AcOEt $=14: 1)$ to give pure $N$ - $(4-$ methylpenta-2,3-dienyl)- $N$-(prop-2-ynyl)tosylamine (3a, 419 mg, 52\%).

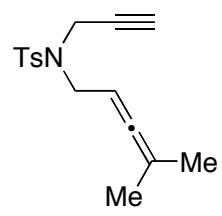

$\boldsymbol{N}$-(4-Methylpenta-2,3-dienyl)- $\boldsymbol{N}$-(prop-2-ynyl)tosylamine (3a). White solid; mp: 56$57{ }^{\circ} \mathrm{C} ;{ }^{1} \mathrm{H}$ NMR $\delta 1.67(\mathrm{~d}, J=3.0 \mathrm{~Hz}, 6 \mathrm{H}), 1.99(\mathrm{t}, J=2.5 \mathrm{~Hz}, 1 \mathrm{H}), 2.42(\mathrm{~s}, 3 \mathrm{H}), 3.79(\mathrm{~d}, J$ $=6.9 \mathrm{~Hz}, 2 \mathrm{H}), 4.16(\mathrm{~d}, J=2.5 \mathrm{~Hz}, 2 \mathrm{H}), 4.81-4.87(\mathrm{~m}, 1 \mathrm{H}), 7.28(\mathrm{~d}, J=8.1 \mathrm{~Hz}, 2 \mathrm{H}), 7.73(\mathrm{~d}$, $J=8.1 \mathrm{~Hz}, 2 \mathrm{H}) ;{ }^{13} \mathrm{C}$ NMR $\delta 20.3,21.5,35.5,46.6,73.3,76.5,83.6,97.0,127.6,129.4$, 136.1, 143.4, 203.8; HRMS (EI) calcd for $\mathrm{C}_{16} \mathrm{H}_{19} \mathrm{NO}_{2} \mathrm{~S}\left(\mathrm{M}^{+}\right)$289.1136, found 289.1136. 
Anal. Calcd for $\mathrm{C}_{16} \mathrm{H}_{19} \mathrm{NO}_{2} \mathrm{~S}: \mathrm{C}, 66.40 ; \mathrm{H}, 6.62 ; \mathrm{N}, 4.84$. Found: C, 66.26; H, 6.61; N, 4.80.

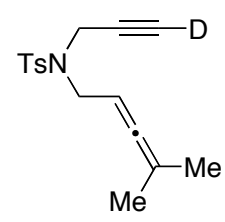

$N$-(4-Methylpenta-2,3-dienyl)- $N$-(prop-2-ynyl-3- $d$ )tosylamine (3a- $d$ ). White solid; mp: $52-53{ }^{\circ} \mathrm{C} ;{ }^{1} \mathrm{H}$ NMR $\delta 1.66(\mathrm{~d}, J=2.7 \mathrm{~Hz}, 6 \mathrm{H}), 2.42(\mathrm{~s}, 3 \mathrm{H}), 3.79(\mathrm{~d}, J=7.2 \mathrm{~Hz}, 2 \mathrm{H}), 4.15(\mathrm{~s}$, $2 \mathrm{H}), 4.83-4.84(\mathrm{~m}, 1 \mathrm{H}), 7.28(\mathrm{~d}, J=8.1 \mathrm{~Hz}, 2 \mathrm{H}), 7.73(\mathrm{~d}, J=8.1 \mathrm{~Hz}, 2 \mathrm{H}) ;{ }^{13} \mathrm{C}$ NMR $\delta 20.3$, 21.6, 35.5, 46.6, 73.6 (t, $J=17.7$ Hz), 76.0, 83.6, 97.1, 127.7, 129.4, 136.1, 143.4, 203.9; HRMS (CI) calcd for $\mathrm{C}_{16} \mathrm{H}_{19} \mathrm{DNO}_{2} \mathrm{~S}\left(\mathrm{M}^{+}+\mathrm{H}\right)$ 291.1276, found 291.1273.

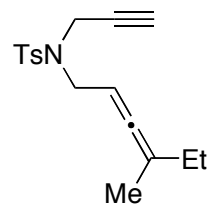

$N$-(4-Methylhexa-2,3-dienyl)- $N$-(prop-2-ynyl)tosylamine (3b). Pale yellow solid; mp: $42-43{ }^{\circ} \mathrm{C} ;{ }^{1} \mathrm{H}$ NMR $\delta 0.97(\mathrm{t}, J=7.4 \mathrm{~Hz}, 3 \mathrm{H}) 1.67(\mathrm{~d}, J=3.0 \mathrm{~Hz}, 3 \mathrm{H}), 1.92(\mathrm{dq}, J=3.0,7.4$ $\mathrm{Hz} 1 \mathrm{H}), 1.98(\mathrm{t}, J=2.4 \mathrm{~Hz}, 1 \mathrm{H}), 2.41(\mathrm{~s}, 3 \mathrm{H}), 3.77(\mathrm{dd}, J=13.8,6.9 \mathrm{~Hz}, 1 \mathrm{H}), 3.83(\mathrm{dd}, J=$ $13.8,6.9 \mathrm{~Hz}, 1 \mathrm{H}), 4.13(\mathrm{dd}, J=18.0,2.4 \mathrm{~Hz}, 1 \mathrm{H}), 4.20(\mathrm{dd}, J=18.0,2.4 \mathrm{~Hz}, 1 \mathrm{H}), 4.90-$ $4.97(\mathrm{~m}, 1 \mathrm{H}), 7.28(\mathrm{~d}, J=8.4 \mathrm{~Hz}, 2 \mathrm{H}), 7.73(\mathrm{~d}, J=8.4 \mathrm{~Hz}, 2 \mathrm{H}) ;{ }^{13} \mathrm{C}$ NMR $\delta 12.0,18.8$, 21.5, 26.8, 35.5, 46.6, 73.4, 76.5, 85.6, 103.3, 127.6, 129.4, 136.1, 143.4, 202.9; HRMS (EI) calcd for $\mathrm{C}_{17} \mathrm{H}_{21} \mathrm{NO}_{2} \mathrm{~S}\left(\mathrm{M}^{+}\right)$303.1293, found 303.1293.

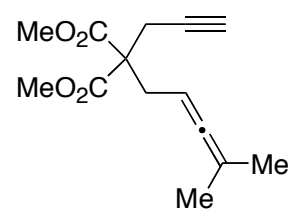

Dimethyl 8-methylnona-6,7-dien-1-yne-4,4-dicarboxylate (3c). Yellow oil; ${ }^{1} \mathrm{H}$ NMR $\delta$ $1.64(\mathrm{~d}, J=3.0 \mathrm{~Hz}, 6 \mathrm{H}), 1.98(\mathrm{t}, J=2.7 \mathrm{~Hz}, 1 \mathrm{H}), 2.70(\mathrm{~d}, J=7.5 \mathrm{~Hz}, 2 \mathrm{H}), 2.85(\mathrm{~d}, J=2.7$ $\mathrm{Hz}, 2 \mathrm{H}), 3.72$ (s, 6H), 4.71-4.80 (m, 1H); ${ }^{13} \mathrm{C}$ NMR $\delta 20.4,22.5,32.5,52.7,57.1,71.2,78.8$, 82.1, 95.4, 170.1, 203.8; HRMS (EI) calcd for $\mathrm{C}_{14} \mathrm{H}_{18} \mathrm{O}_{4}\left(\mathrm{M}^{+}\right)$250.1205, found 250.1212. 


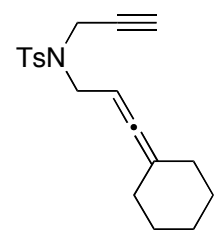

$N$-(3-Cyclohexylideneprop-2-enyl)- $N$-(prop-2-ynyl)tosylamine (3e). White solid; mp: 79-80 ${ }^{\circ} \mathrm{C} ;{ }^{1} \mathrm{H}$ NMR $\delta 1.42-1.62(\mathrm{~m}, 6 \mathrm{H}), 1.98(\mathrm{t}, J=2.4 \mathrm{~Hz}, 1 \mathrm{H}), 2.06-2.10(\mathrm{~m}, 4 \mathrm{H}), 2.42(\mathrm{~s}$, $3 \mathrm{H}), 3.80(\mathrm{~d}, J=6.9 \mathrm{~Hz}, 2 \mathrm{H}), 4.16(\mathrm{~d}, J=2.4 \mathrm{~Hz}, 2 \mathrm{H}), 4.81-4.86(\mathrm{~m}, 1 \mathrm{H}), 7.29$ (d, $J=8.4$ $\mathrm{Hz}, 2 \mathrm{H}), 7.73(\mathrm{~d}, J=8.4 \mathrm{~Hz}, 2 \mathrm{H}) ;{ }^{13} \mathrm{C}$ NMR $\delta 21.5,25.8,27.1,31.2,35.4,46.7,73.3,76.4$, 83.2, 104.1, 127.5, 129.3, 136.0, 143.3, 200.6; HRMS (EI) calcd for $\mathrm{C}_{19} \mathrm{H}_{23} \mathrm{NO}_{2} \mathrm{~S}\left(\mathrm{M}^{+}\right)$ 329.1449 , found 329.1451 .

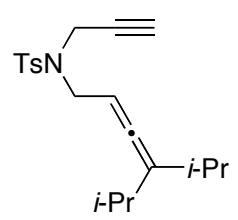

$N$-(4-Isopropyl-5-methylhexa-2,3-dienyl)- $N$-(prop-2-ynyl)tosylamine (3f). White solid; mp: 55-56 ${ }^{\circ} \mathrm{C} ;{ }^{1} \mathrm{H}$ NMR $\delta 0.99(\mathrm{~d}, J=7.1 \mathrm{~Hz}, 12 \mathrm{H}), 1.97(\mathrm{t}, J=2.4 \mathrm{~Hz}, 1 \mathrm{H}), 2.15$ (dsep, $J$ $=2.4,7.1 \mathrm{~Hz}, 2 \mathrm{H}), 2.42(\mathrm{~s}, 3 \mathrm{H}), 3.81(\mathrm{~d}, J=6.9 \mathrm{~Hz}, 2 \mathrm{H}), 4.20(\mathrm{~d}, J=2.4 \mathrm{~Hz}, 2 \mathrm{H}), 5.10(\mathrm{tt}$, $J=7.0,2.1 \mathrm{~Hz}, 1 \mathrm{H}), 7.29(\mathrm{~d}, J=8.1 \mathrm{~Hz}, 2 \mathrm{H}), 7.74(\mathrm{~d}, J=8.1 \mathrm{~Hz}, 2 \mathrm{H}) ;{ }^{13} \mathrm{C} \mathrm{NMR} \delta 21.5$, $22.1,22.4,29.5,35.6,46.8,73.7,89.7,120.1,127.7,129.4,136.2$, 143.4, 200.9; HRMS (CI) calcd for $\mathrm{C}_{20} \mathrm{H}_{28} \mathrm{NO}_{2} \mathrm{~S}\left(\mathrm{M}^{+}+\mathrm{H}\right) 346.1841$, found 346.1837 .

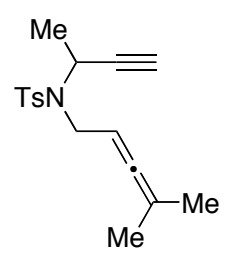

$\boldsymbol{N}$-(4-Methylpenta-2,3-dienyl)- $\boldsymbol{N}$-(1-methylprop-2-ynyl)tosylamine (3g). White solid; mp: 58-59 ${ }^{\circ} \mathrm{C} ;{ }^{1} \mathrm{H}$ NMR $\delta 1.49(\mathrm{~d}, J=7.0 \mathrm{~Hz}, 3 \mathrm{H}), 1.67(\mathrm{~d}, J=2.7 \mathrm{~Hz}, 6 \mathrm{H}), 2.12(\mathrm{~d}, J=2.2$ $\mathrm{Hz}, 1 \mathrm{H}), 2.41$ (s, 3H), $3.68(\mathrm{dd}, J=15.6,8.0 \mathrm{~Hz}, 1 \mathrm{H}), 3.90(\mathrm{dd}, J=15.6,5.3 \mathrm{~Hz}, 1 \mathrm{H}), 4.88$ $(\mathrm{dq}, J=7.0,2.2 \mathrm{~Hz}, 1 \mathrm{H}), 5.03-5.11(\mathrm{~m}, 1 \mathrm{H}), 7.27(\mathrm{~d}, J=8.1 \mathrm{~Hz}, 2 \mathrm{H}), 7.72(\mathrm{~d}, J=8.1 \mathrm{~Hz}$, $2 \mathrm{H}) ;{ }^{13} \mathrm{C}$ NMR $\delta 19.8,20.2,21.5,22.6,44.8,45.9,73.0,81.5,87.8,97.0,127.5,129.4$, 136.5, 143.2, 202.0; HRMS (EI) calcd for $\mathrm{C}_{17} \mathrm{H}_{21} \mathrm{NO}_{2} \mathrm{~S}\left(\mathrm{M}^{+}\right)$303.1293, found 303.1306. 


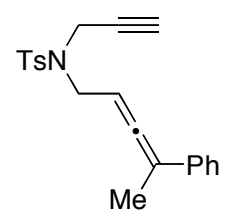

$\boldsymbol{N}$-(4-Phenylpenta-2,3-dienyl)- $\boldsymbol{N}$-(prop-2-ynyl)tosylamine (3h). Pale yellow oil; ${ }^{1} \mathrm{H}$ NMR $\delta 1.99(\mathrm{t}, J=2.6 \mathrm{~Hz}, 1 \mathrm{H}) 2.89(\mathrm{~d}, J=2.7 \mathrm{~Hz}, 3 \mathrm{H}), 2.41(\mathrm{~s}, 3 \mathrm{H}), 3.90(\mathrm{dd}, J=14.4,7.2 \mathrm{~Hz}$, $1 \mathrm{H}), 4.01(\mathrm{dd}, J=14.4,6.6 \mathrm{~Hz}, 1 \mathrm{H}), 4.16(\mathrm{dd}, J=18.0,2.6 \mathrm{~Hz}, 1 \mathrm{H}), 4.25(\mathrm{dd}, J=15.9$, $2.6 \mathrm{~Hz}, 1 \mathrm{H}), 5.33-5.40(\mathrm{~m}, 1 \mathrm{H}), 7.19-7.39(\mathrm{~m}, 7 \mathrm{H}), 7.74(\mathrm{~d}, J=8.1 \mathrm{~Hz}, 2 \mathrm{H}) ;{ }^{13} \mathrm{C} \mathrm{NMR}$ $\delta 17.0,21.5,36.0,46.0,73.8,76.4,88.1,102.6,125.8,127.0,127.6,128.3,129.5,136.0$, 136.1, 143.5, 205.8; HRMS (EI) calcd for $\mathrm{C}_{21} \mathrm{H}_{21} \mathrm{NO}_{2} \mathrm{~S}\left(\mathrm{M}^{+}\right)$351.1293, found 351.1297.

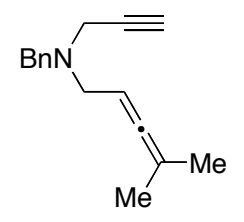

$N$-(4-Methylpenta-2,3-dienyl)- $N$-(but-3-ynyl)benzylamine (3i). Yellow oil; ${ }^{1} \mathrm{H}$ NMR $\delta$ $1.65(\mathrm{~d}, J=2.7 \mathrm{~Hz}, 6 \mathrm{H}), 2.17(\mathrm{t}, J=2.6 \mathrm{~Hz}, 1 \mathrm{H}), 3.09(\mathrm{~d}, J=6.9 \mathrm{~Hz}, 2 \mathrm{H}), 3.30(\mathrm{~d}, J=2.6$ $\mathrm{Hz}, 2 \mathrm{H}), 3.63(\mathrm{~s}, 2 \mathrm{H}), 4.12-4.98(\mathrm{~m}, 1 \mathrm{H}), 7.19-7.33(\mathrm{~m}, 5 \mathrm{H}) ;{ }^{13} \mathrm{C}$ NMR $\delta 20.5,41.3,53.6$, 56.7, 73.0, 78.7, 85.9, 95.3, 127.0, 128.2, 129.1, 138.5, 203.3; HRMS (EI) calcd for $\mathrm{C}_{16} \mathrm{H}_{19} \mathrm{~N}\left(\mathrm{M}^{+}\right) 225.1517$, found 225.1517.

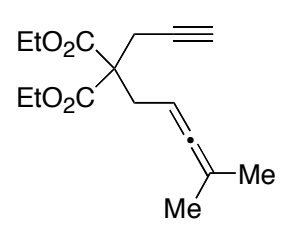

Diethyl 8-methylnona-6,7-dien-1-yne-4,4-dicarboxylate (3j). Yellow oil; ${ }^{1} \mathrm{H}$ NMR $\delta 1.25$ $(\mathrm{t}, J=7.1 \mathrm{~Hz}, 6 \mathrm{H}), 1.65(\mathrm{~d}, J=3.0 \mathrm{~Hz}, 6 \mathrm{H}), 1.98(\mathrm{t}, J=2.7 \mathrm{~Hz}, 1 \mathrm{H}), 2.71(\mathrm{~d}, J=7.8 \mathrm{~Hz}$, $2 \mathrm{H}), 2.85(\mathrm{~d}, J=2.7 \mathrm{~Hz}, 2 \mathrm{H}), 4.12-4.25(\mathrm{~m}, 4 \mathrm{H}), 4.72-4.81(\mathrm{~m}, 1 \mathrm{H}) ;{ }^{13} \mathrm{C}$ NMR $\delta$ 14.0, 20.4, 22.3, 32.3, 57.0, 61.5, 71.1, 78.9, 82.2, 95.2, 169.6, 203.9; HRMS (EI) calcd for $\mathrm{C}_{16} \mathrm{H}_{22} \mathrm{O}_{4}$ $\left(\mathrm{M}^{+}\right)$278.1518, found 278.1516.

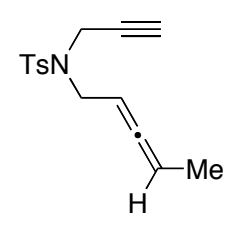


$\boldsymbol{N}$-(Penta-2,3-dienyl)- $\boldsymbol{N}$-(prop-2-ynyl)tosylamine (3k). White solid; mp: $45-46{ }^{\circ} \mathrm{C} ;{ }^{1} \mathrm{H}$ NMR $\delta 1.63(\mathrm{dd}, J=7.1,3.0 \mathrm{~Hz}, 3 \mathrm{H}), 2.00(\mathrm{t}, J=2.7 \mathrm{~Hz}, 1 \mathrm{H}), 2.40(\mathrm{~s}, 3 \mathrm{H}), 3.78$ (ddd, $J=$ 21.2, 7.2, $2.2 \mathrm{~Hz}, 1 \mathrm{H}), 3.86(\mathrm{ddd}, J=21.2,7.2,2.2 \mathrm{~Hz}, 1 \mathrm{H}), 4.11(\mathrm{dd}, J=18.3,2.5 \mathrm{~Hz}, 1 \mathrm{H})$, $4.18(\mathrm{dd}, J=18.3,2.5 \mathrm{~Hz}, 1 \mathrm{H}), 4.90-4.98(\mathrm{~m}, 1 \mathrm{H}), 5.10-5.18(\mathrm{~m}, 1 \mathrm{H}), 7.27$ (d, $J=8.4 \mathrm{~Hz}$, $2 \mathrm{H}), 7.71(\mathrm{~d}, J=8.4 \mathrm{~Hz}, 2 \mathrm{H}) ;{ }^{13} \mathrm{C}$ NMR $\delta 14.0,21.5,35.7,46.2,73.5,76.4,85.2,87.4$, 127.6, 129.4, 136.0, 143.5, 206.5; HRMS (EI) calcd for $\mathrm{C}_{15} \mathrm{H}_{17} \mathrm{NO}_{2} \mathrm{~S}\left(\mathrm{M}^{+}\right)$275.0980, found 275.0974 .

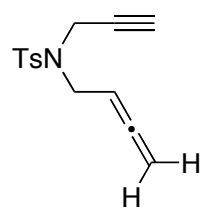

$N$-(Buta-2,3-dienyl)- $N$-(prop-2-ynyl)tosylamine (3l). White solid; mp: $55-56{ }^{\circ} \mathrm{C} ;{ }^{1} \mathrm{H}$ NMR $\delta 2.03(\mathrm{t}, J=2.4 \mathrm{~Hz}, 1 \mathrm{H}), 2.42(\mathrm{~s}, 3 \mathrm{H}), 3.88(\mathrm{dt}, J=6.8,2.5 \mathrm{~Hz}, 2 \mathrm{H}), 4.15(\mathrm{~d}, J=2.4 \mathrm{~Hz}$, $2 \mathrm{H}$ ), 4.78 (dt, $J=6.8,2.5 \mathrm{~Hz}, 2 \mathrm{H}), 5.04$ (quint, $J=6.8 \mathrm{~Hz}, 1 \mathrm{H}), 7.29$ (d, $J=8.3 \mathrm{~Hz}, 2 \mathrm{H}$ ), $7.73(\mathrm{~d}, J=8.3 \mathrm{~Hz}, 2 \mathrm{H}) ;{ }^{13} \mathrm{C}$ NMR $\delta 21.5,35.8,45.6,73.7,76.4,85.3,127.6,129.5,136.0$, 143.6, 209.7; HRMS (EI) calcd for $\mathrm{C}_{14} \mathrm{H}_{15} \mathrm{NO}_{2} \mathrm{~S}\left(\mathrm{M}^{+}\right)$261.0823, found 261.0825.

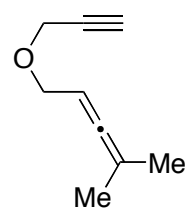

4-Methylpent-2,3-dienyl prop-2-ynyl ether (3m). Yellow oil; ${ }^{1} \mathrm{H}$ NMR $\delta 1.71$ (d, $J=3.0$ $\mathrm{Hz}, 6 \mathrm{H}), 2.41$ (t, $J=2.5 \mathrm{~Hz}, 1 \mathrm{H}), 4.04(\mathrm{~d}, J=6.9 \mathrm{~Hz}, 2 \mathrm{H}), 4.17$ (d, $J=2.5 \mathrm{~Hz}, 2 \mathrm{H}), 5.01-$ $5.07(\mathrm{~m}, 1 \mathrm{H}) ;{ }^{13} \mathrm{C}$ NMR $\delta$ 20.3, 56.3, 68.5, 74.1, 79.7, 85.4, 96.1, 203.4; HRMS (EI) calcd for $\mathrm{C}_{9} \mathrm{H}_{12} \mathrm{O}\left(\mathrm{M}^{+}\right)$136.0888, found 136.0886.

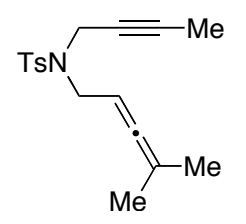

$\boldsymbol{N}$-(4-Methylpent-2,3-dienyl)- $\boldsymbol{N}$-(but-2-ynyl)tosylamine (3n). White solid; mp: $42-43{ }^{\circ} \mathrm{C}$; ${ }^{1} \mathrm{H}$ NMR $\delta 1.54(\mathrm{t}, J=2.4 \mathrm{~Hz}, 3 \mathrm{H}), 1.66(\mathrm{~d}, J=2.7 \mathrm{~Hz}, 6 \mathrm{H}) 2.40(\mathrm{~s}, 3 \mathrm{H}), 3.75(\mathrm{~d}, J=6.6$ 
$\mathrm{Hz}, 2 \mathrm{H}), 4.07(\mathrm{q}, J=2.4 \mathrm{~Hz}, 2 \mathrm{H}), 4.82-4.84(\mathrm{~m}, 1 \mathrm{H}), 7.27(\mathrm{~d}, J=8.3 \mathrm{~Hz}, 2 \mathrm{H}), 7.72(\mathrm{~d}, J=$ $8.3 \mathrm{~Hz}, 2 \mathrm{H}) ;{ }^{13} \mathrm{C}$ NMR $\delta 3.2,20.4,21.5,36.1,46.5,71.6,81.1,83.9,96.8,127.7,129.2$, 136.2, 143.0, 203.6; HRMS (EI) calcd for $\mathrm{C}_{17} \mathrm{H}_{21} \mathrm{NO}_{2} \mathrm{~S}\left(\mathrm{M}^{+}\right)$303.1293, found 303.1290.

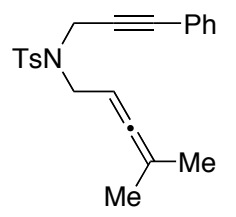

$\boldsymbol{N}$-(4-Methylpent-2,3-dienyl)- $\mathbf{N}$-(3-phenylprop-2-ynyl)tosylamine (3o). White solid; mp: $58-59{ }^{\circ} \mathrm{C} ;{ }^{1} \mathrm{H}$ NMR $\delta 1.70(\mathrm{~d}, J=2.7 \mathrm{~Hz}, 6 \mathrm{H}), 2.36(\mathrm{~s}, 3 \mathrm{H}), 3.88(\mathrm{~d}, J=6.9 \mathrm{~Hz}, 2 \mathrm{H}), 4.41(\mathrm{~s}$, 2H), 4.91-4.97 (m, 1H), $7.04(\mathrm{~d}, J=8.1 \mathrm{~Hz}, 2 \mathrm{H}), 7.23-7.33(\mathrm{~m}, 5 \mathrm{H}), 7.71(\mathrm{~d}, J=8.1 \mathrm{~Hz}$, $2 \mathrm{H}) ;{ }^{13} \mathrm{C}$ NMR $\delta 20.3,21.4,36.5,46.8,81.7,83.7,85.3,97.0,122.3,127.7,128.0,128.2$, 129.4, 131.4, 135.9, 143.3, 203.9; HRMS (EI) calcd for $\mathrm{C}_{22} \mathrm{H}_{23} \mathrm{NO}_{2} \mathrm{~S}\left(\mathrm{M}^{+}\right) 365.1449$, found 365.1457.

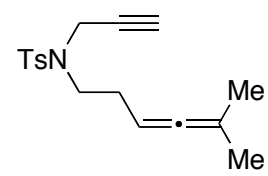

$\mathrm{N}$-(5-Methylhex-3,4-dienyl)- $\mathrm{N}$-(prop-2-ynyl)tosylamine (3p). Pale yellow oil; ${ }^{1} \mathrm{H}$ NMR $\delta$ $1.67(\mathrm{~d}, J=3.0 \mathrm{~Hz}, 6 \mathrm{H}), 2.02(\mathrm{t}, J=2.4 \mathrm{~Hz}, 1 \mathrm{H}), 2.22(\mathrm{dt}, J=7.5,7.5 \mathrm{~Hz}, 2 \mathrm{H}), 2.42$ (s, 2H), 3.25 (t, $J=7.5 \mathrm{~Hz}, 2 \mathrm{H}), 4.15(\mathrm{~d}, J=2.4 \mathrm{~Hz}, 2 \mathrm{H}), 4.85-4.94(\mathrm{~m}, 1 \mathrm{H}), 7.29(\mathrm{~d}, J=8.3 \mathrm{~Hz}$, 2H), $7.73(\mathrm{~d}, J=8.3 \mathrm{~Hz}, 2 \mathrm{H}) ;{ }^{13} \mathrm{C}$ NMR $\delta 20.6,21.6,27.8,36.3,46.0,73.6,76.6,85.0,95.9$, 127.6, 129.4, 136.0, 143.4, 202.4; HRMS (EI) calcd for $\mathrm{C}_{17} \mathrm{H}_{21} \mathrm{NO}_{2} \mathrm{~S}\left(\mathrm{M}^{+}\right) 303.1293$, found 303.1299 .

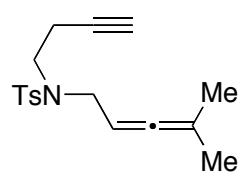

$\mathrm{N}$-(4-Methylpent-2,3-dienyl)- $\mathrm{N}$-(but-3-ynyl)tosylamine (3q). Pale yellow solid; mp: 62$63{ }^{\circ} \mathrm{C} ;{ }^{1} \mathrm{H}$ NMR $\delta 1.65(\mathrm{~d}, J=2.7 \mathrm{~Hz}, 6 \mathrm{H}), 1.96(\mathrm{t}, J=2.7 \mathrm{~Hz}, 1 \mathrm{H}), 2.41(\mathrm{~s}, 3 \mathrm{H}), 2.41-2.50$ $(\mathrm{m}, 2 \mathrm{H}), 3.03-3.35(\mathrm{~m}, 2 \mathrm{H}), 3.78(\mathrm{~d}, J=7.2 \mathrm{~Hz}, 2 \mathrm{H}), 4.71-4.80(\mathrm{~m}, 1 \mathrm{H}), 7.29(\mathrm{~d}, J=8.4 \mathrm{~Hz}$, 2H), $7.76(\mathrm{~d}, J=8.4 \mathrm{~Hz}, 2 \mathrm{H}) ;{ }^{13} \mathrm{C}$ NMR $\delta 19.0,20.3,21.5,45.6,48.3,70.0,80.9,84.2,97.1$, 127.1, 129.7, 136.9, 143.2, 203.5; HRMS (EI) calcd for $\mathrm{C}_{17} \mathrm{H}_{21} \mathrm{NO}_{2} \mathrm{~S}\left(\mathrm{M}^{+}\right) 303.1293$ found, 
303.1296.

General Procedure for RCM of Allenynes. To a solution of Schrock complex (27.7 mg, $0.036 \mathrm{mmol})$ in dry toluene $(4.0 \mathrm{~mL})$ was added a solution of diethyl 8-methyl nona-2,3diene-1-yne-4,4-dicarboxylate $(\mathbf{3 j}, 67.1 \mathrm{mg}, 0.241 \mathrm{mmol})$ in dry toluene $(4.8 \mathrm{~mL})$. After being stirred under argon atmosphere for $3 \mathrm{~h}$ at room temperature, the volatile material was removed under reduced pressure. The residue was purified by preparative thin-layer chromatography (hexane:AcOEt $=5: 1$ ) to give pure diethyl 3-(3-methylbuta-1,2dienyl)cyclopent-3-ene-1,1-dicarboxylate (4j, $63.9 \mathrm{mg}, 95 \%)$.

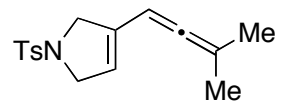

3-(3-Methylbuta-1,2-dienyl)-1-tosyl-3-pyrroline (4a). Pale yellow solid; mp: $114-115{ }^{\circ} \mathrm{C}$; ${ }^{1} \mathrm{H}$ NMR $\delta 1.70(\mathrm{~d}, J=2.7 \mathrm{~Hz}, 6 \mathrm{H}), 2.42(\mathrm{~s}, 3 \mathrm{H}), 4.05-4.07(\mathrm{~m}, 2 \mathrm{H}), 4.13-4.16(\mathrm{~m}, 2 \mathrm{H})$, $5.42(\mathrm{t}, J=1.8 \mathrm{~Hz}, 1 \mathrm{H}), 5.69(\mathrm{t}, J=2.9 \mathrm{~Hz}, 1 \mathrm{H}), 7.32(\mathrm{~d}, J=8.3 \mathrm{~Hz}, 2 \mathrm{H}), 7.72(\mathrm{~d}, J=8.3$ $\mathrm{Hz}, 2 \mathrm{H}) ;{ }^{13} \mathrm{C}$ NMR $\delta 20.4,21.5,54.8,55.5,86.1,98.6,119.2,127.4,129.7,134.3,135.1$, 143.3, 204.2; HRMS (EI) calcd for $\mathrm{C}_{16} \mathrm{H}_{19} \mathrm{NO}_{2} \mathrm{~S}\left(\mathrm{M}^{+}\right)$289.1136, found 289.1138. Anal. Calcd for $\mathrm{C}_{16} \mathrm{H}_{19} \mathrm{NO}_{2} \mathrm{~S}$ : C, 66.40; H, 6.62; N, 4.84. Found: C, 66.50; H, 6.64; N, 4.80 .

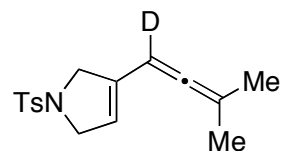

3-(3-Methylbuta-1,2-dienyl-1-d)-1-tosyl-3-pyrroline (4a-d). Pale yellow solid; mp: 112$113{ }^{\circ} \mathrm{C} ;{ }^{1} \mathrm{H}$ NMR $\delta 1.71(\mathrm{~s}, 6 \mathrm{H}), 2.43(\mathrm{~s}, 3 \mathrm{H}), 4.04-4.07(\mathrm{~m}, 2 \mathrm{H}), 4.14-4.17(\mathrm{~m}, 2 \mathrm{H}), 5.42(\mathrm{t}$, $J=1.8 \mathrm{~Hz}, 1 \mathrm{H}), 7.32(\mathrm{~d}, J=8.4 \mathrm{~Hz}, 2 \mathrm{H}), 7.72(\mathrm{~d}, J=8.4 \mathrm{~Hz}, 2 \mathrm{H}) ;{ }^{13} \mathrm{C}$ NMR $\delta 20.4,21.5$, 54.8, 55.5, 86.1, 98.6, 119.1, 127.4, 129.7, 134.2, 135.1, 143.3, 204.1; HRMS (EI) calcd for $\mathrm{C}_{16} \mathrm{H}_{18} \mathrm{DNO}_{2} \mathrm{~S}\left(\mathrm{M}^{+}\right) 290.1198$, found 290.1199 .

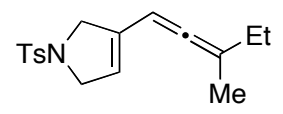

3-(3-Methylpenta-1,2-dienyl)-1-tosyl-3-pyrroline (4b). Yellow solid; mp: 88-89 ${ }^{\circ} \mathrm{C} ;{ }^{1} \mathrm{H}$ NMR $\delta 0.95(\mathrm{t}, J=7.5 \mathrm{~Hz}, 3 \mathrm{H}), 1.71(\mathrm{~d}, J=2.7 \mathrm{~Hz}, 3 \mathrm{H}), 1.98(\mathrm{dq}, J=3.3,7.5 \mathrm{~Hz}, 2 \mathrm{H})$, 
$2.42(\mathrm{~s}, 3 \mathrm{H}), 4.03-4.06(\mathrm{~m}, 2 \mathrm{H}), 4.13-4.17(\mathrm{~m}, 2 \mathrm{H}), 5.42(\mathrm{t}, J=2.0 \mathrm{~Hz}, 1 \mathrm{H}), 5.79(\mathrm{q}, J=3.0$ $\mathrm{Hz}, 1 \mathrm{H}), 7.32(\mathrm{~d}, J=8.4 \mathrm{~Hz}, 2 \mathrm{H}), 7.72(\mathrm{~d}, J=8.4 \mathrm{~Hz}, 2 \mathrm{H}) ;{ }^{13} \mathrm{C}$ NMR $\delta 12.2,19.0,21.5$, 26.9, 54.9, 55.5, 88.1, 104.9, 119.0, 127.4, 129.7, 134.1, 135.1, 143.4, 203.5; HRMS (EI) calcd for $\mathrm{C}_{17} \mathrm{H}_{21} \mathrm{NO}_{2} \mathrm{~S}\left(\mathrm{M}^{+}\right)$303.1293, found 303.1290.

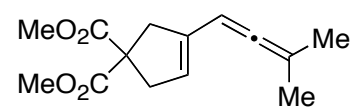

Dimethyl 3-(3-Methylbuta-1,2-dienyl)cyclopent-3-ene-1,1-dicarboxylate (4c). Pale yellow solid; mp: 62-63 ${ }^{\circ} \mathrm{C} ;{ }^{1} \mathrm{H}$ NMR $\delta 1.72(\mathrm{~d}, J=3.0 \mathrm{~Hz}, 6 \mathrm{H}), 2.96-3.02(\mathrm{~m}, 2 \mathrm{H}), 3.09(\mathrm{q}$, $J=2.0 \mathrm{~Hz}, 2 \mathrm{H}), 3.74(\mathrm{~s}, 6 \mathrm{H}), 5.42(\mathrm{t}, J=2.1 \mathrm{~Hz}, 1 \mathrm{H}), 5.79(\mathrm{t}, J=2.9 \mathrm{~Hz}, 1 \mathrm{H}) ;{ }^{13} \mathrm{C}$ NMR $\delta$ 20.5, 41.0, 41.2, 52.8, 58.6, 88.4, 97.5, 122.6, 137.1, 172.6, 204.1; HRMS (EI) calcd for $\mathrm{C}_{14} \mathrm{H}_{18} \mathrm{O}_{4}\left(\mathrm{M}^{+}\right) 250.1205$, found 250.1212 .

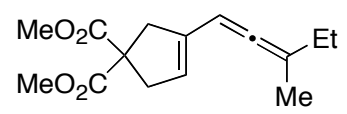

Dimethyl 3-(3-Methylpenta-1,2-dienyl)cyclopent-3-ene-1,1-dicarboxylate (4d). ${ }^{1} \mathrm{H}$ NMR $\delta 0.99$ (t, $J=7.5 \mathrm{~Hz}, 3 \mathrm{H}), 1.72(\mathrm{~d}, J=2.7 \mathrm{~Hz}, 3 \mathrm{H}), 1.99(\mathrm{dq}, J=3.3,7.2 \mathrm{~Hz}, 2 \mathrm{H})$, 2.991-2.996 (m, 2H), 3.087-3.094 (m, 2H), $3.74(\mathrm{~s}, 6 \mathrm{H}), 5.38-5.42(\mathrm{~m}, 1 \mathrm{H})$, 5.86-5.92 (m, $1 \mathrm{H})$.

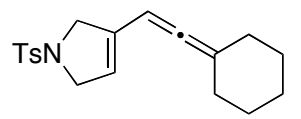

3-(2-Cyclohexylidenevinyl)-1-tosyl-3-pyrroline (4e). Yellow solid; mp: 101-102 ${ }^{\circ} \mathrm{C} ;{ }^{1} \mathrm{H}$ NMR $\delta 1.40-1.60(\mathrm{~m}, 6 \mathrm{H}), 2.09-2.11(\mathrm{~m}, 4 \mathrm{H}), 2.42(\mathrm{~s}, 3 \mathrm{H}), 4.05-4.07(\mathrm{~m}, 2 \mathrm{H}), 4.14-4.17$ $(\mathrm{m}, 2 \mathrm{H}), 5.41(\mathrm{t}, J=1.8 \mathrm{~Hz}, 1 \mathrm{H}), 5.70(\mathrm{~s}, 1 \mathrm{H}), 7.32(\mathrm{~d}, J=8.1 \mathrm{~Hz}, 2 \mathrm{H}), 7.72(\mathrm{~d}, J=8.1 \mathrm{~Hz}$, $2 \mathrm{H}) ;{ }^{13} \mathrm{C}$ NMR $\delta 21.5,25.9,27.3,31.3,54.8,55.6,85.9,105.7,118.9,127.4,129.7,134.2$, 135.1, 143.3, 200.9; HRMS (CI) calcd for $\mathrm{C}_{19} \mathrm{H}_{24} \mathrm{NO}_{2} \mathrm{~S}\left(\mathrm{M}^{+}+\mathrm{H}\right)$ 330.1528, found 330.1524 .

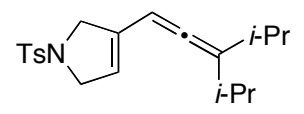

3-(3-Isopropyl-4-methylpenta-1,2-dienyl)-1-tosyl-3-pyrroline (4f). Yellow solid; mp: 75$76{ }^{\circ} \mathrm{C} ;{ }^{1} \mathrm{H}$ NMR $\delta 0.98(\mathrm{~d}, J=6.6 \mathrm{~Hz}, 6 \mathrm{H}), 1.00(\mathrm{~d}, J=6.6 \mathrm{~Hz}, 6 \mathrm{H}), 2.19(\mathrm{dsep}, J=2.1,6.6$ 
$\mathrm{Hz}, 2 \mathrm{H}), 2.42(\mathrm{~s}, 3 \mathrm{H}), 4.01-4.04(\mathrm{~m}, 2 \mathrm{H}), 4.15-4.18(\mathrm{~m}, 2 \mathrm{H}), 5.41(\mathrm{t}, J=2.0 \mathrm{~Hz}, 1 \mathrm{H}), 5.93$ (s, $1 \mathrm{H}), 7.30(\mathrm{~d}, J=8.3 \mathrm{~Hz}, 2 \mathrm{H}), 7.70(\mathrm{~d}, J=8.3 \mathrm{~Hz}, 2 \mathrm{H}) ;{ }^{13} \mathrm{C}$ NMR $\delta 21.5,22.2,22.4,29.9$, 54.7, 55.7, 91.5, 118.8, 121.3, 127.4, 129.7, 134.0, 135.0, 143.4, 201.9; HRMS (CI) calcd for $\mathrm{C}_{20} \mathrm{H}_{28} \mathrm{NO}_{2} \mathrm{~S}\left(\mathrm{M}^{+}+\mathrm{H}\right) 346.1841$, found 346.1840 .

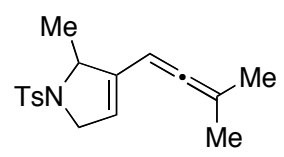

2-Methyl-3-(3-methylbuta-1,2-dienyl)-1-tosyl-3-pyrroline (4g). Yellow solid; mp: 89$90{ }^{\circ} \mathrm{C} ;{ }^{1} \mathrm{H}$ NMR $\delta 1.42(\mathrm{~d}, J=6.3 \mathrm{~Hz}, 3 \mathrm{H}), 1.72(\mathrm{dd}, J=8.3,2.9 \mathrm{~Hz}, 6 \mathrm{H}), 2.41(\mathrm{~s}, 3 \mathrm{H}), 4.07$ $(\mathrm{dt}, J=15.6,2.1 \mathrm{~Hz}, 1 \mathrm{H}), 4.18(\mathrm{dq}, J=15.6,2.1 \mathrm{~Hz}, 1 \mathrm{H}), 4.51(\mathrm{t}, J=6.3 \mathrm{~Hz}, 1 \mathrm{H}), 5.35$ (s, $1 \mathrm{H}), 5.63(\mathrm{t}, J=3.0 \mathrm{~Hz}, 1 \mathrm{H}), 7.29(\mathrm{~d}, J=8.1 \mathrm{~Hz}, 2 \mathrm{H}), 7.70(\mathrm{~d}, J=8.1 \mathrm{~Hz}, 2 \mathrm{H}) ;{ }^{13} \mathrm{C}$ NMR $\delta$ 20.0, 20.3, 21.5, 22.1, 54.4, 63.1, 85.9, 98.2, 118.9, 127.2, 129.6, 135.3, 139.8, 143.2, 204.2; HRMS (CI) calcd for $\mathrm{C}_{17} \mathrm{H}_{22} \mathrm{NO}_{2} \mathrm{~S}\left(\mathrm{M}^{+}+\mathrm{H}\right) 304.1371$, found 304.1290 .

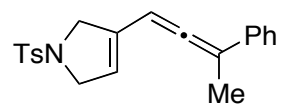

3-(3-Phenylbuta-1,2-dienyl)-1-tosyl-3-pyrroline (4h). Yellow solid; mp: $144-145{ }^{\circ} \mathrm{C} ;{ }^{1} \mathrm{H}$ NMR $\delta 2.13(\mathrm{~d}, J=2.7 \mathrm{~Hz}, 3 \mathrm{H}), 2.43(\mathrm{~s}, 3 \mathrm{H}), 4.08-4.11(\mathrm{~m}, 2 \mathrm{H}), 4.18-4.22(\mathrm{~m}, 2 \mathrm{H}), 5.58(\mathrm{t}$, $J=2.1 \mathrm{~Hz}, 1 \mathrm{H}), 5.79(\mathrm{~d}, J=2.7 \mathrm{~Hz}, 1 \mathrm{H}), 7.22-7.34(\mathrm{~m}, 7 \mathrm{H}), 7.70(\mathrm{~d}, J=8.4 \mathrm{~Hz}, 2 \mathrm{H}) ;{ }^{13} \mathrm{C}$ NMR $\delta 17.3,21.8,54.9,55.8,90.3,104.2,121.0,126.1,127.5,127.6,128.6,130.0,134.3$, 134.4, 136.0, 143.6, 207.6; HRMS (EI) calcd for $\mathrm{C}_{21} \mathrm{H}_{21} \mathrm{NO}_{2} \mathrm{~S}\left(\mathrm{M}^{+}\right)$351.1293, found 351.1295 .

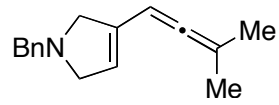

1-Benzyl-3-(3-methylbuta-1,2-dienyl)-3-pyrroline (4i). Yellow oil; ${ }^{1} \mathrm{H}$ NMR $\delta 1.68$ (d, $J$ $=2.7 \mathrm{~Hz}, 6 \mathrm{H}), 3.47-3.48(\mathrm{~m}, 2 \mathrm{H}), 3.52-3.56(\mathrm{~m}, 2 \mathrm{H}), 3.82(\mathrm{~s}, 2 \mathrm{H}), 5.60(\mathrm{t}, J=1.8 \mathrm{~Hz}, 1 \mathrm{H})$, $5.81(\mathrm{t}, J=2.7 \mathrm{~Hz}, 1 \mathrm{H}), 7.23-7.39(\mathrm{~m}, 5 \mathrm{H}) ;{ }^{13} \mathrm{C}$ NMR $\delta 20.5,59.9,60.5,60.6,87.1,97.4$, 122.5, 126.9, 128.3, 128.6, 137.1, 139.5, 204.0; HRMS (EI) calcd for $\mathrm{C}_{16} \mathrm{H}_{19} \mathrm{~N}\left(\mathrm{M}^{+}\right)$ 225.1517, found 225.1521 .

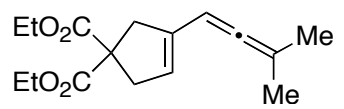


Diethyl 3-(3-Methylbuta-1,2-dienyl)cyclopent-3-ene-1,1-dicarboxylate (4j). Yellow oil; ${ }^{1} \mathrm{H}$ NMR $\delta 1.24(\mathrm{t}, J=7.2 \mathrm{~Hz}, 6 \mathrm{H}), 1.72(\mathrm{~d}, J=2.7 \mathrm{~Hz}, 6 \mathrm{H}), 2.97-2.98(\mathrm{~m}, 2 \mathrm{H}), 3.06-3.07$ $(\mathrm{m}, 2 \mathrm{H}), 4.19(\mathrm{q}, J=7.2 \mathrm{~Hz}, 4 \mathrm{H}), 4.13(\mathrm{t}, J=8.1 \mathrm{~Hz}, 1 \mathrm{H}), 5.41(J=2.1 \mathrm{~Hz}, 1 \mathrm{H}), 5.79(\mathrm{t}, J$ $=2.7 \mathrm{~Hz}, 1 \mathrm{H}) ;{ }^{13} \mathrm{C}$ NMR $\delta$ 14.0, 20.6, 40.9, 41.1, 58.7, 61.5, 88.4, 97.5, 122.7, 137.0, 172.1, 204.1; HRMS (EI) calcd for $\mathrm{C}_{16} \mathrm{H}_{22} \mathrm{O}_{4}\left(\mathrm{M}^{+}\right)$278.1518, found 278.1517.

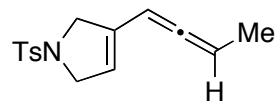

3-(Buta-1,2-dienyl)-1-tosyl-3-pyrroline (4k). Yelow solid; mp: $121-122{ }^{\circ} \mathrm{C} ;{ }^{1} \mathrm{H}$ NMR $\delta$ $1.69(\mathrm{dd}, J=7.1,3.1 \mathrm{~Hz}, 3 \mathrm{H}), 2.43$ (s, 3H), 4.07-4.08 (m, 2H), 4.15-4.27 (m, 2H), 5.37 (quint, $J=6.6 \mathrm{~Hz}, 1 \mathrm{H}), 5.46(\mathrm{~d}, J=1.2 \mathrm{~Hz}, 1 \mathrm{H}), 5.80$ (sext, $J=3.2 \mathrm{~Hz}, 1 \mathrm{H}), 7.32(\mathrm{~d}, J=$ $8.1 \mathrm{~Hz}, 2 \mathrm{H}), 7.73(\mathrm{~d}, J=8.1 \mathrm{~Hz}, 2 \mathrm{H}) ;{ }^{13} \mathrm{C}$ NMR $\delta$ 14.2, 21.5, 54.7, 55.5, 87.6, 89.1, 119.9, 127.4, 129.7, 134.1, 134.6, 143.4, 207.1; HRMS (EI) calcd for $\mathrm{C}_{15} \mathrm{H}_{17} \mathrm{NO}_{2} \mathrm{~S}\left(\mathrm{M}^{+}\right)$275.0980, found 275.0981 .

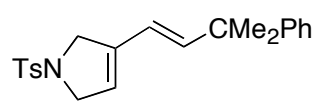

3-(3-Methyl-3-phenylbut-1-enyl)-1-tosyl-3-pyrroline (5). Yellow oil; ${ }^{1} \mathrm{H}$ NMR $\delta 1.40$ (s, $6 \mathrm{H}), 2.42(\mathrm{~s}, 3 \mathrm{H}), 4.17(\mathrm{~s}, 4 \mathrm{H}), 5.51(\mathrm{~s}, 1 \mathrm{H}), 5.68(\mathrm{~d}, J=16.2 \mathrm{~Hz}, 1 \mathrm{H}), 6.06$ (d, J = $16.2 \mathrm{~Hz}$, $1 \mathrm{H}), 7.28-7.33(\mathrm{~m}, 7 \mathrm{H}), 7.73(\mathrm{~d}, J=8.4 \mathrm{~Hz}, 2 \mathrm{H}) ;{ }^{13} \mathrm{C}$ NMR $\delta 21.5,28.5,40.7,53.8,55.0$, $77.2,119.8,121.4,126.0,127.4,128.2,129.8,134.2,137.1,143.3,143.4,147.9$ [one sp $^{2}$ carbon signal is missing due to overlapping]; HRMS (EI) calcd for $\mathrm{C}_{22} \mathrm{H}_{25} \mathrm{NO}_{2} \mathrm{~S}\left(\mathrm{M}^{+}\right)$ 367.5054 , found 367.1604 .

\section{Diels-Alder Reaction of Vinylallene 4c and Maleic Anhydride ${ }^{3}$}

To a stirred solution of vinylallene $4 \mathbf{c}(23.5 \mathrm{mg}, 0.093 \mathrm{mmol})$ in dry toluene $(0.30 \mathrm{~mL})$ at room temperature was added maleic anhydride $(18.2 \mathrm{mg}, 0.186 \mathrm{mmol})$. After 4 days, the solvent was removed, and the residue was purified by preparative thin-layer chromatography (hexane:AcOEt = 1:1) to give the Diels-Alder adduct $6(22.5 \mathrm{mg}, 70 \%)$.

(3) (a) Regás, D.; Afonso, M. M.; Rodríguez, M. L; Palenzuela, J. A. J. Org. Chem. 2003, 68, 7845. (b) Bentz, D.; Laschat, S. Synthesis, 2000, 1766. 


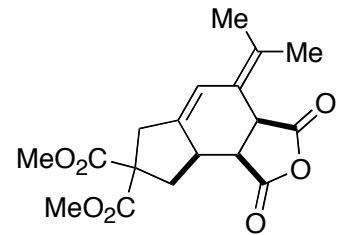

Dimethyl 4-isopropylidene-1,3-dioxo-1,3,3a,4,6,8,8a,8b-octahydroindeno[4,5-c]furan7,7-dicarboxylate (6). White solid; $145-146{ }^{\circ} \mathrm{C} ;{ }^{1} \mathrm{H}$ NMR $\delta 1.81$ (s, 3H), 1.99 (s, 3H), 2.69 $(\mathrm{dd}, J=11.0,7.5 \mathrm{~Hz}, 1 \mathrm{H}), 2.76(\mathrm{~m}, 1 \mathrm{H}), 2.89(\mathrm{dd}, J=11.0,10.5 \mathrm{~Hz}, 1 \mathrm{H}), 3.05(\mathrm{~s}, 2 \mathrm{H}), 3.62$ $(\mathrm{dd}, J=9.2,5.8 \mathrm{~Hz}, 1 \mathrm{H}), 4.22(\mathrm{~d}, J=5.8 \mathrm{~Hz}, 1 \mathrm{H}), 6.32(\mathrm{~s}, 1 \mathrm{H}) ;{ }^{13} \mathrm{C}$ NMR $\delta 21.6,21.9,34.9$, 37.3, 38.8, 44.2, 44.6, 52.93, 52.95, 59.7, 119.3, 119.8, 134.9, 141.4, 170.5, 170.9, 171.4, 171.9; HRMS (EI) calcd for $\mathrm{C}_{18} \mathrm{H}_{20} \mathrm{O}_{7}\left(\mathrm{M}^{+}\right)$348.1209, found 348.1208. 

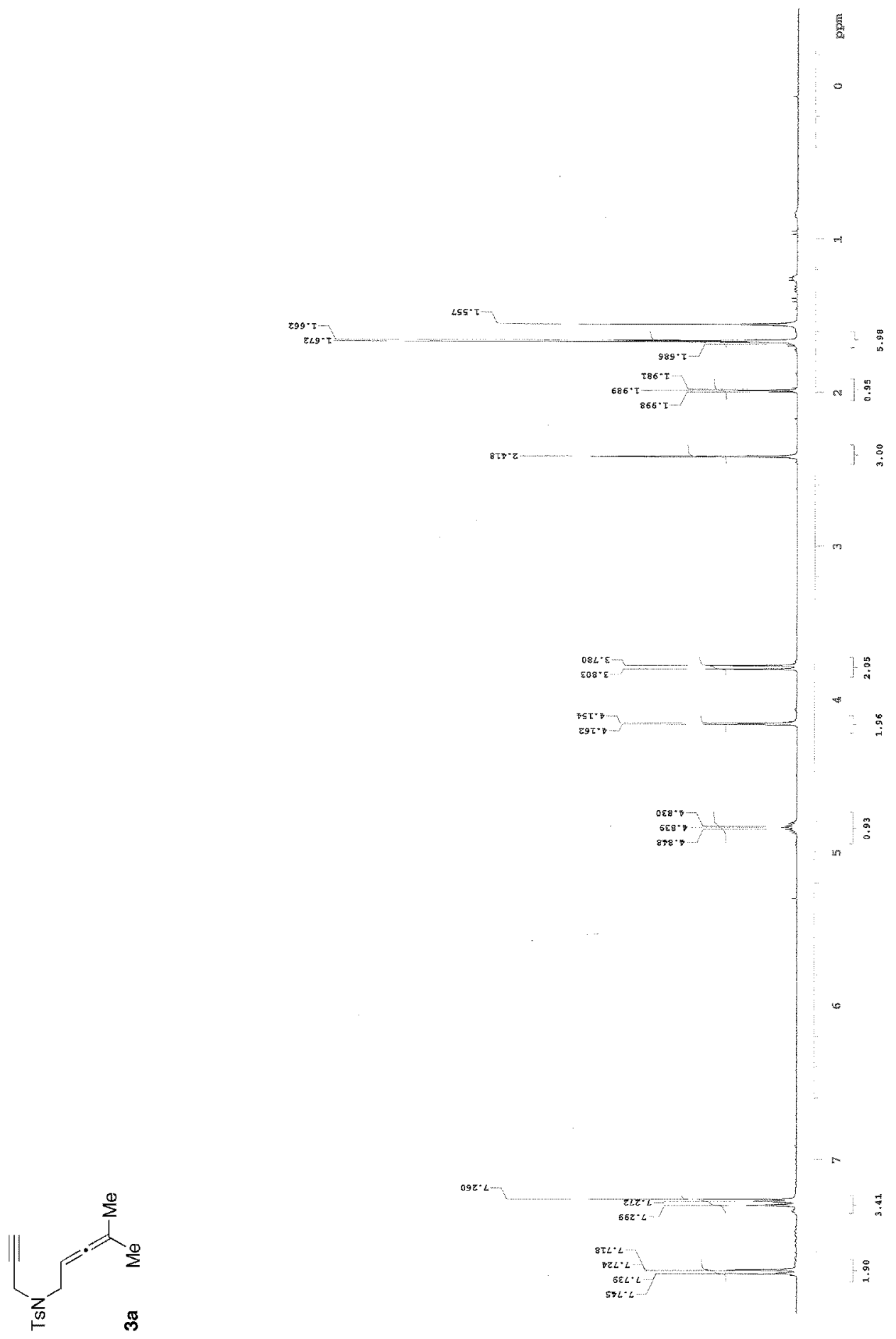


$$
\text { 丰 }
$$




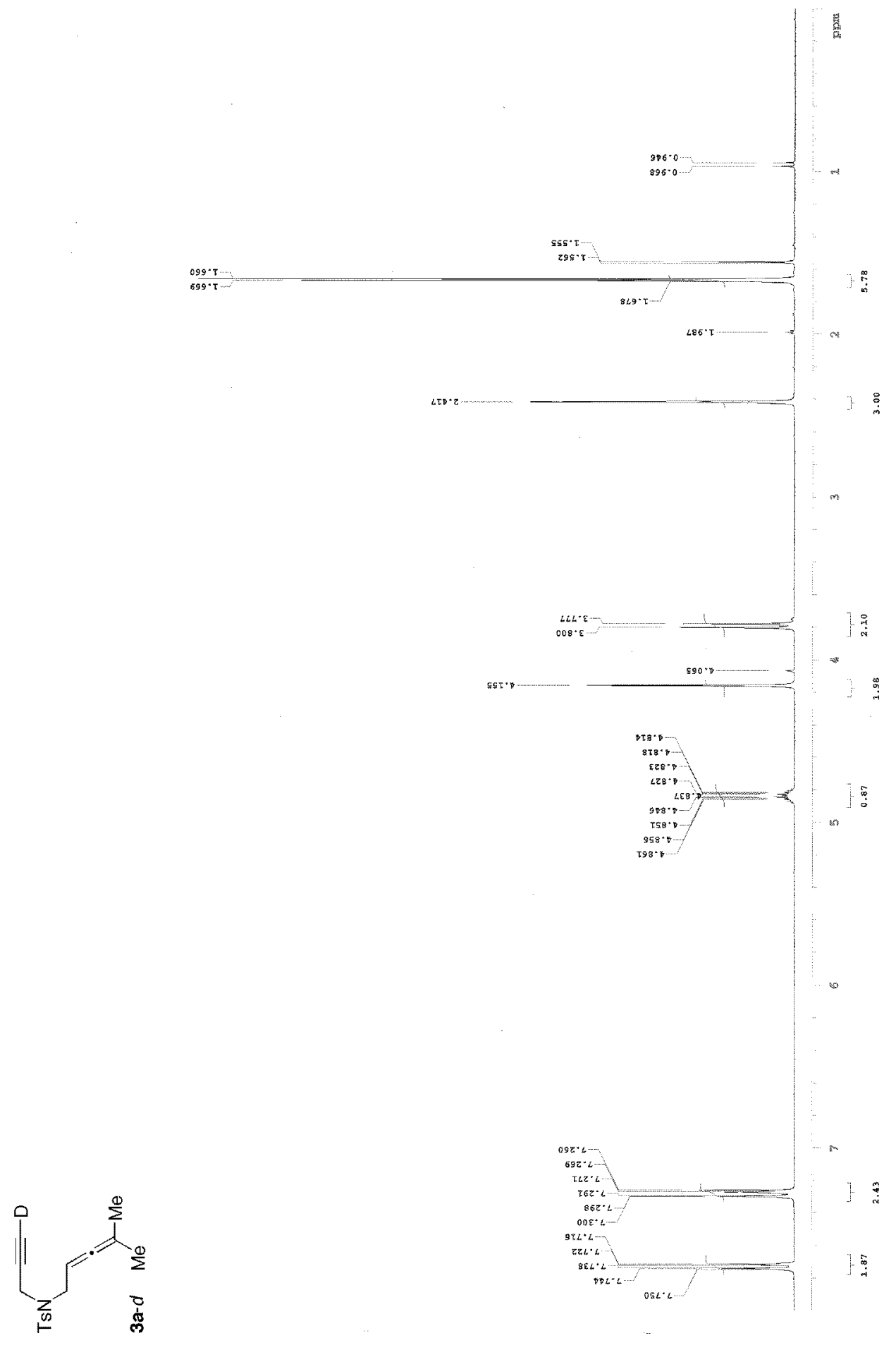




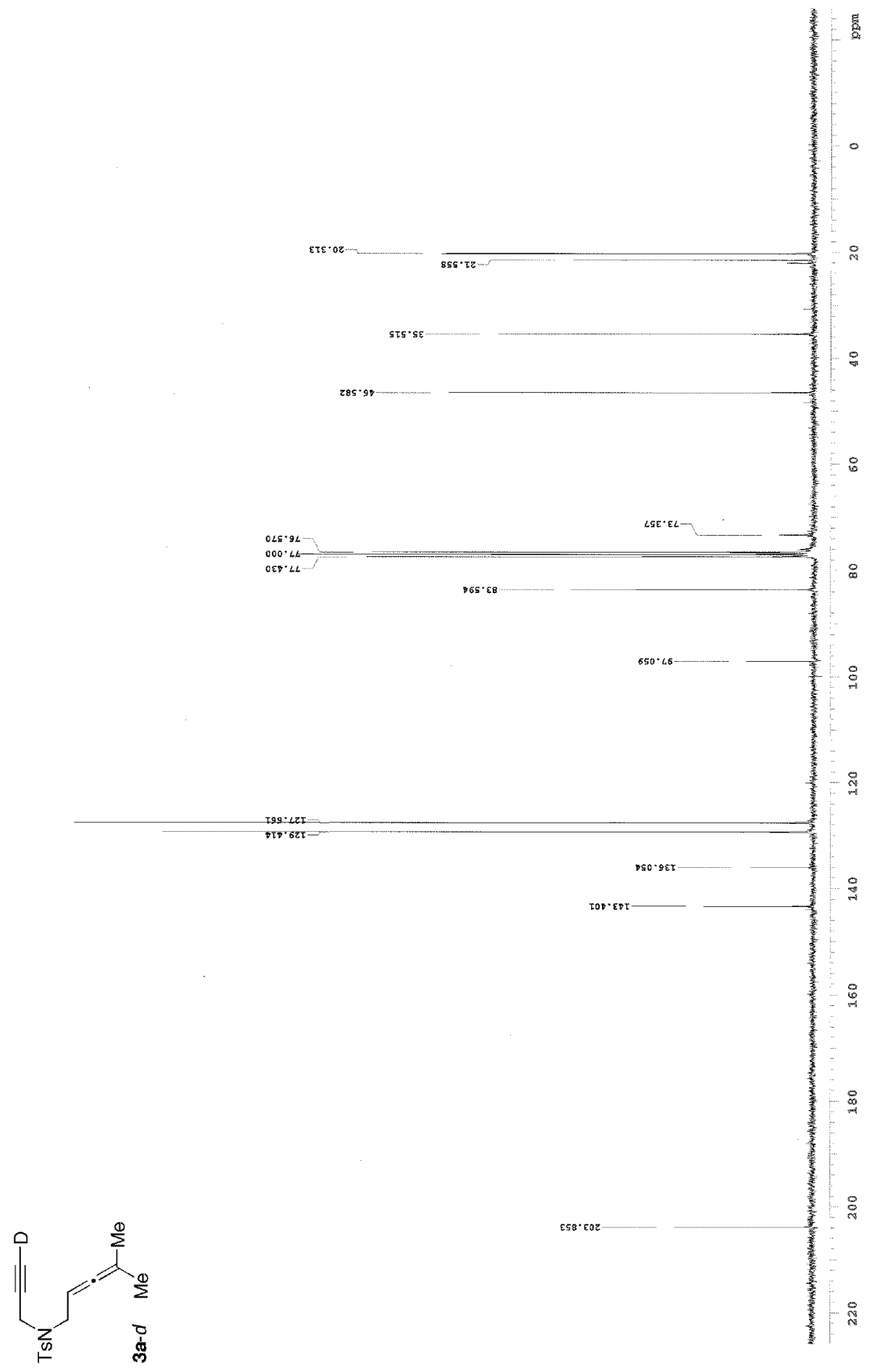




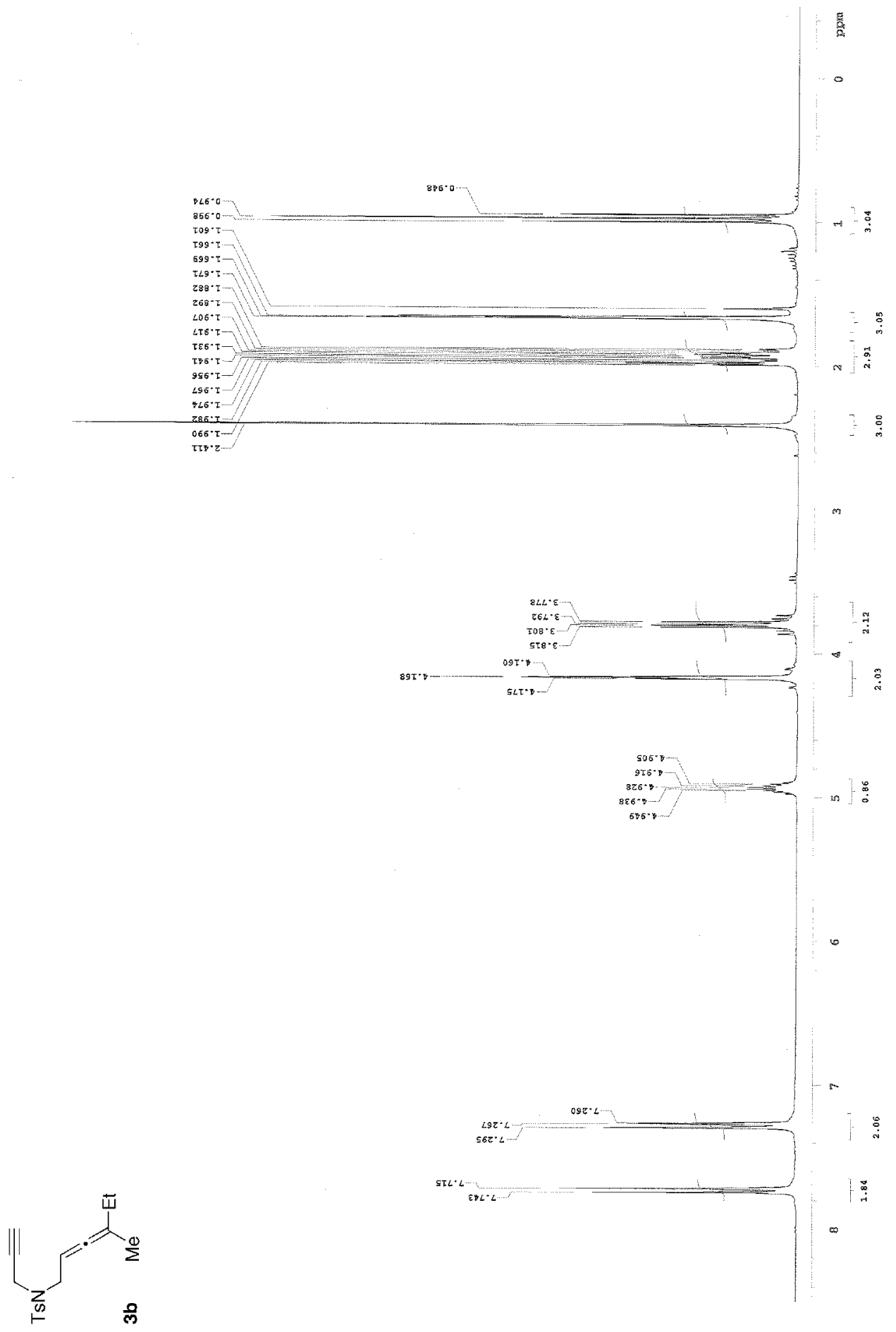




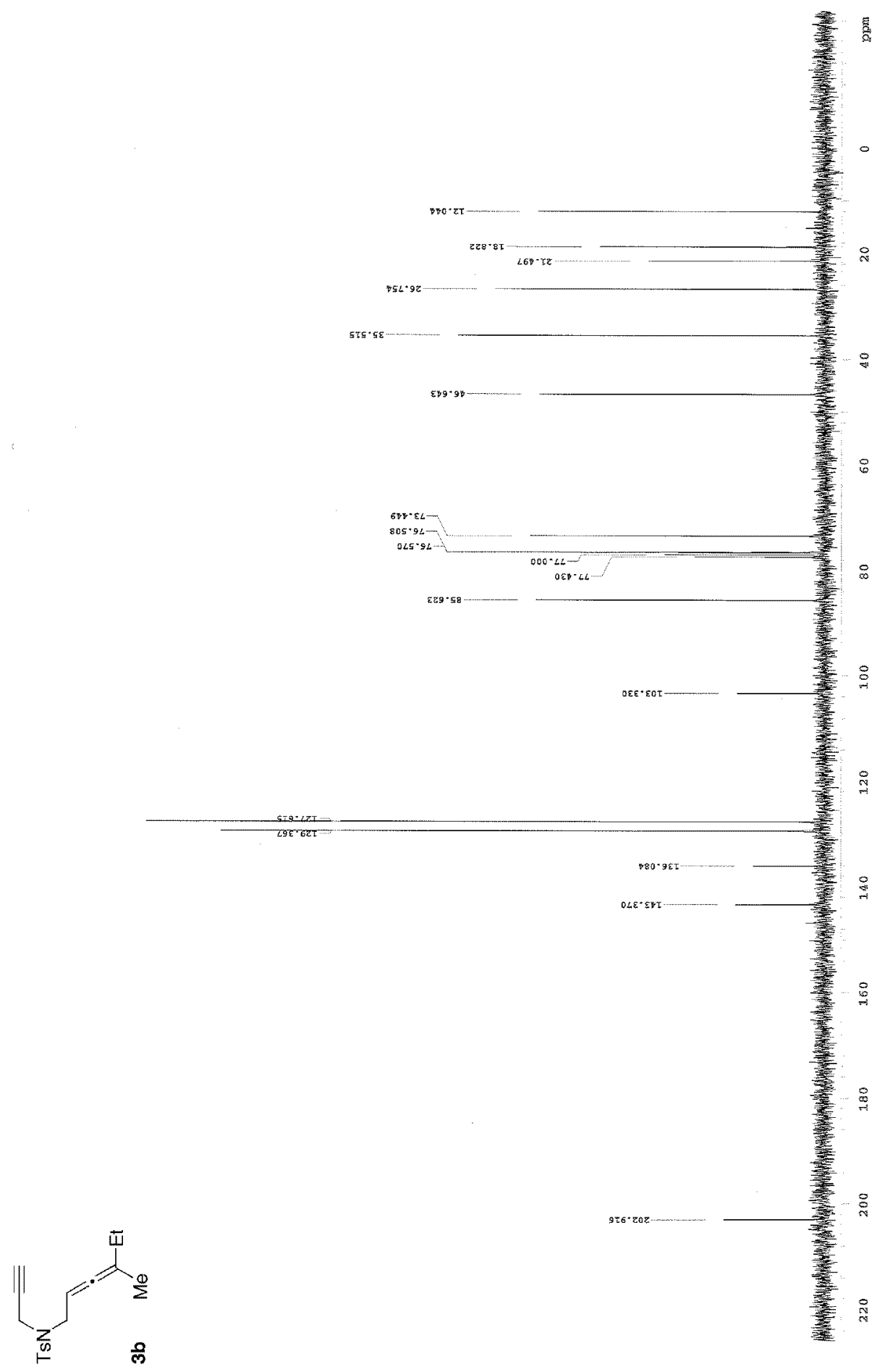




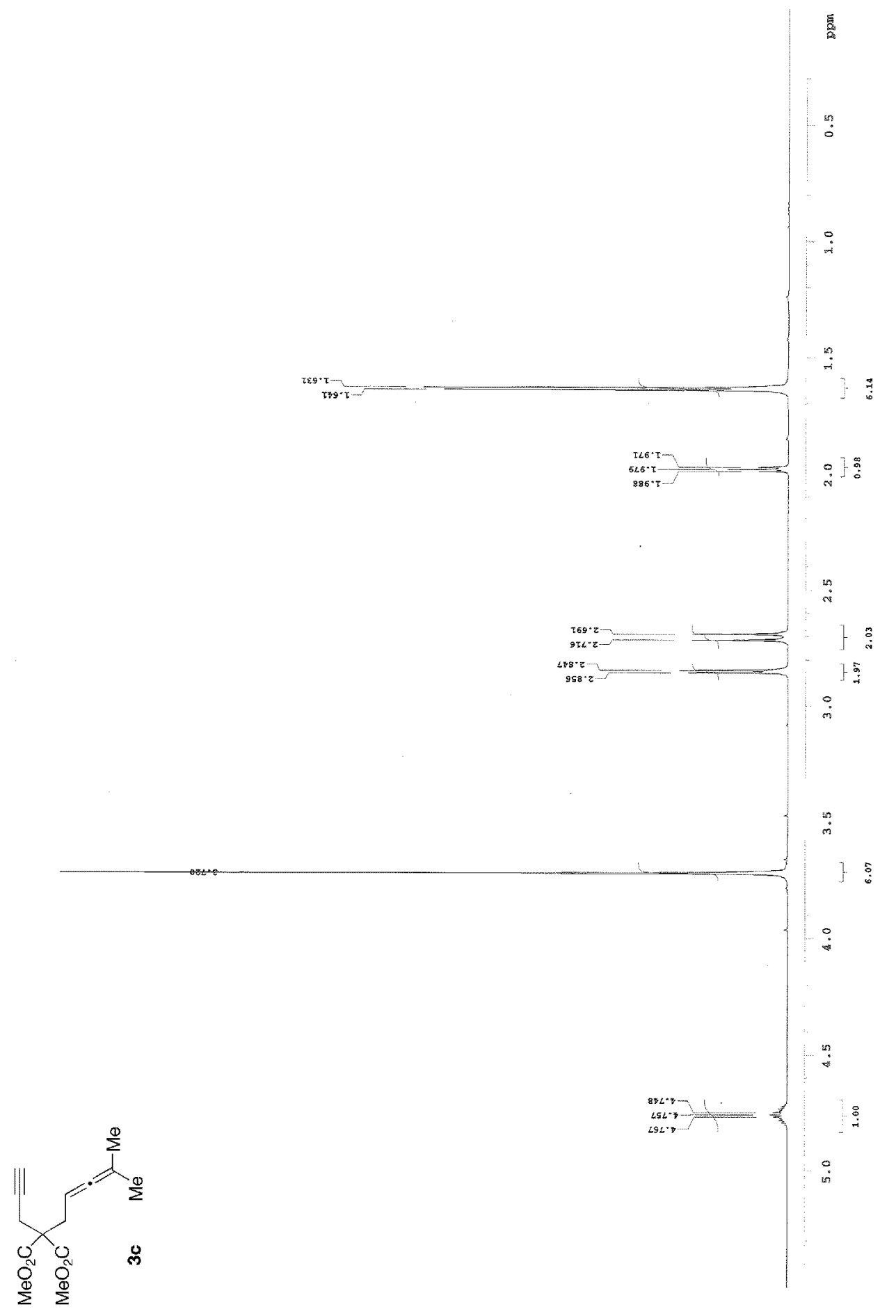




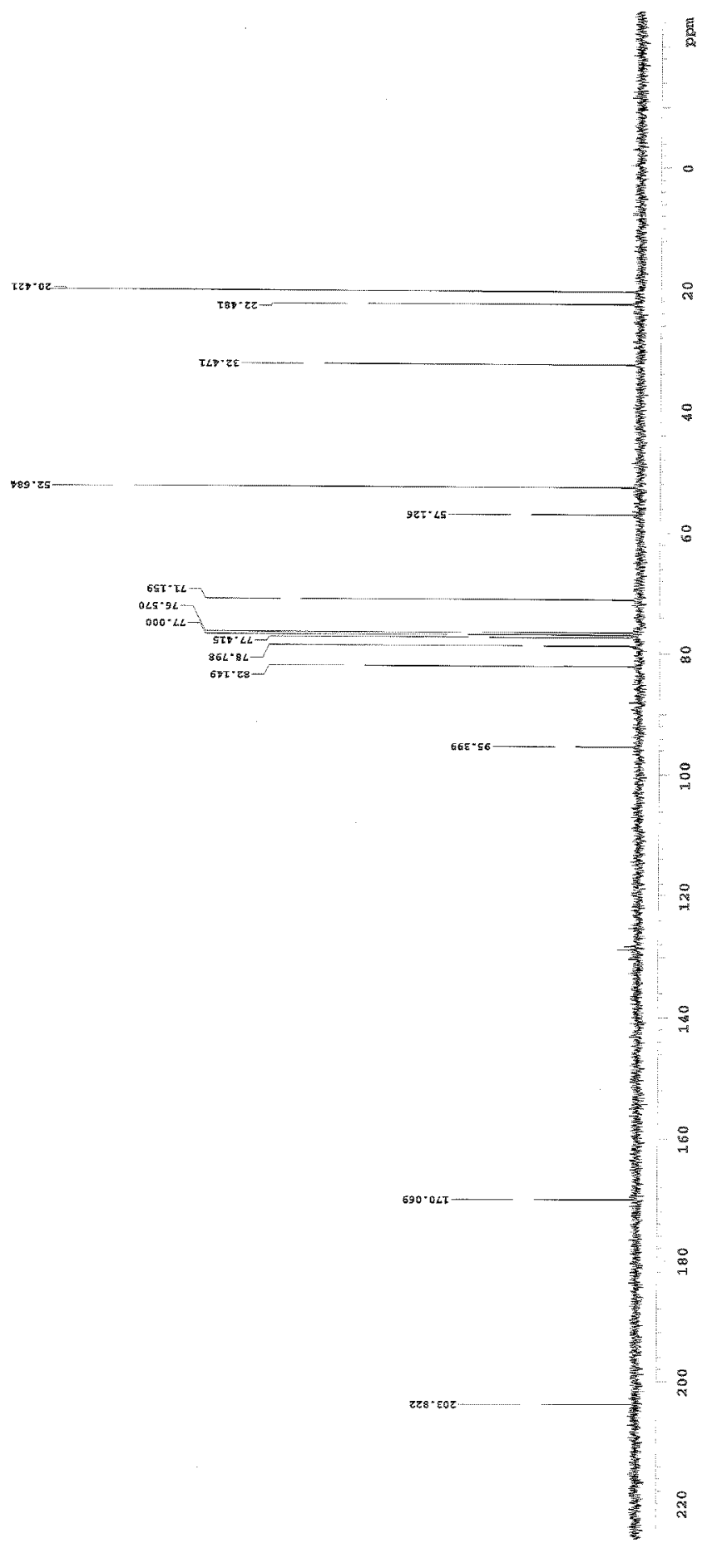




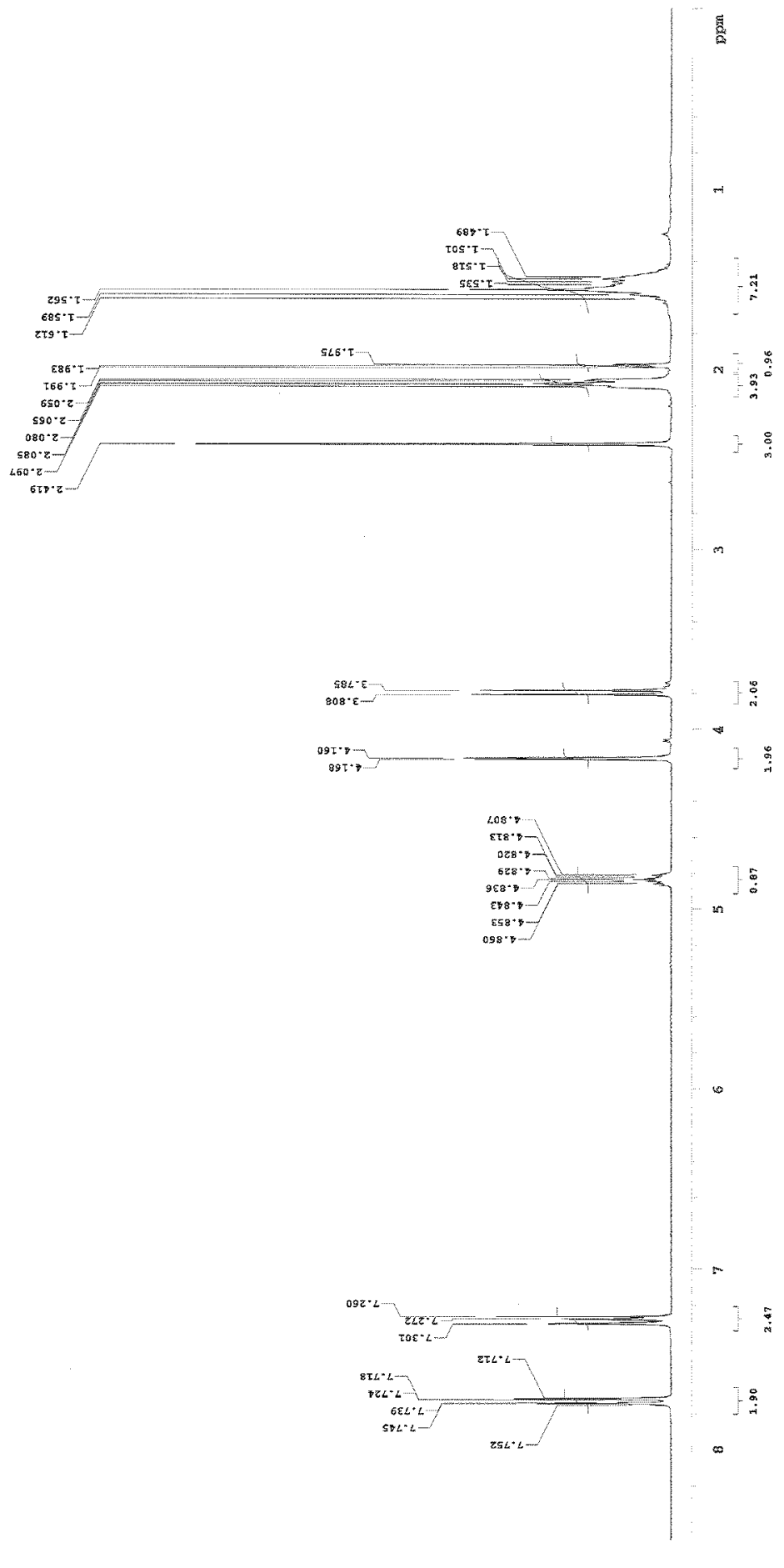




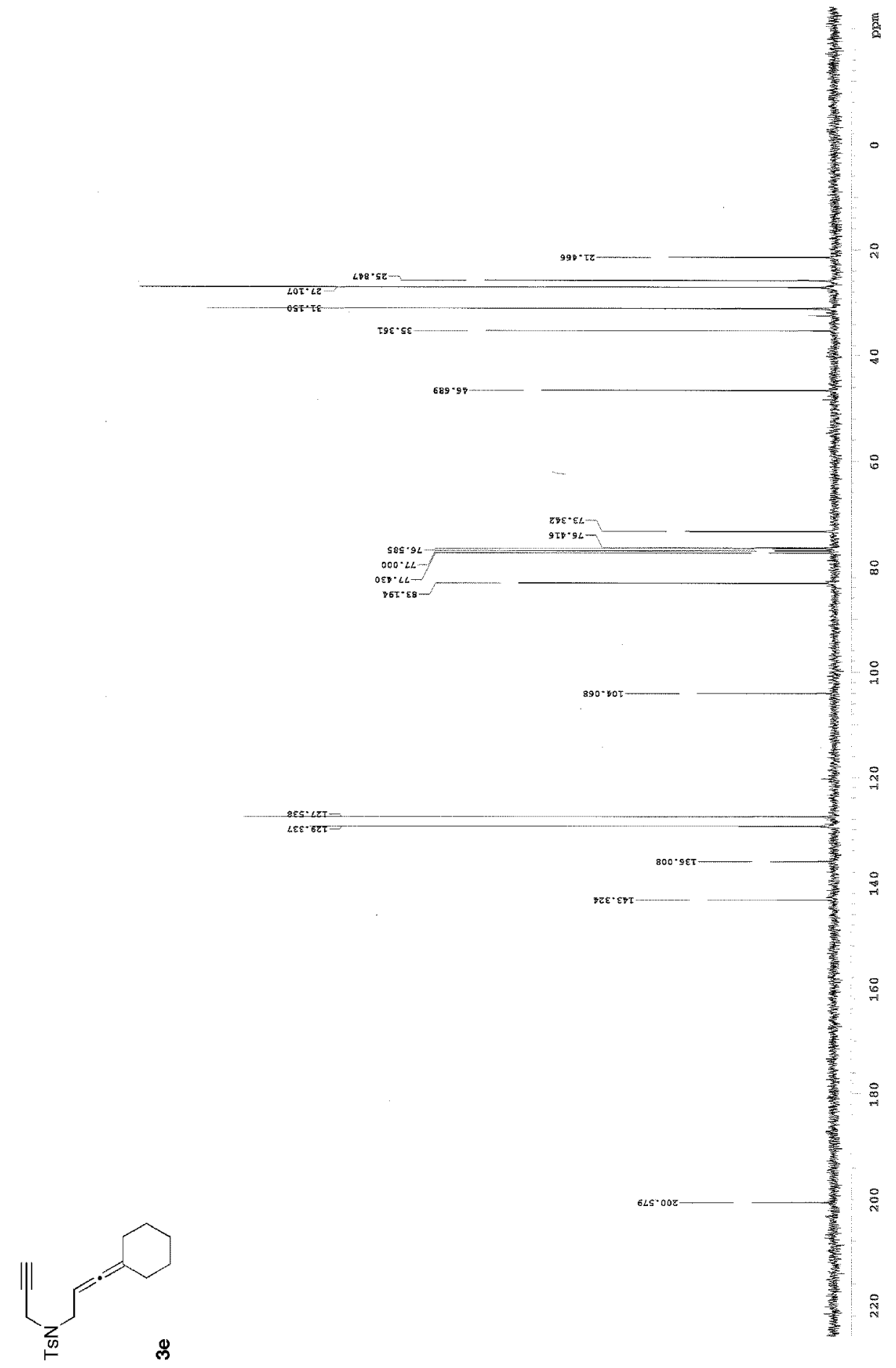




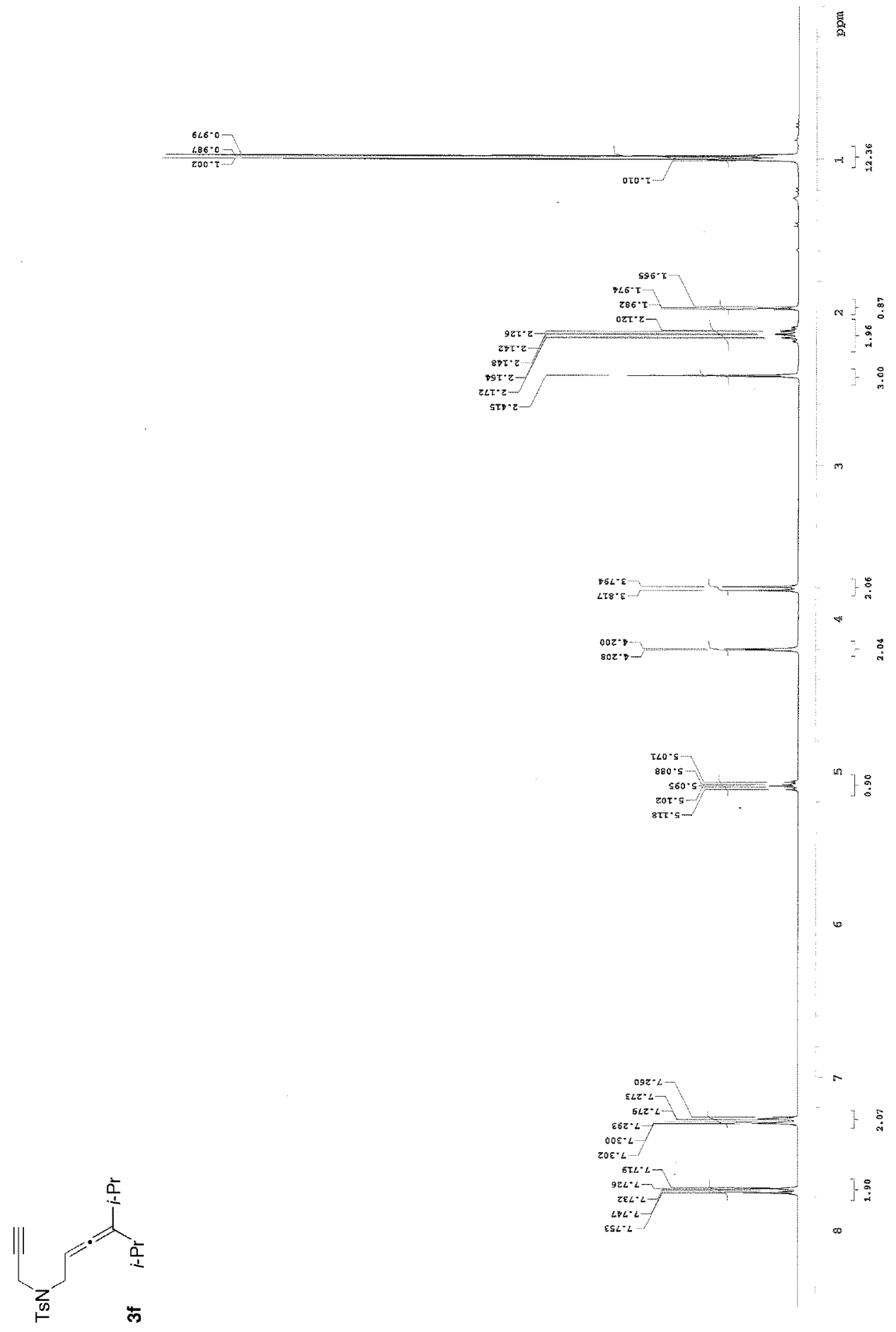




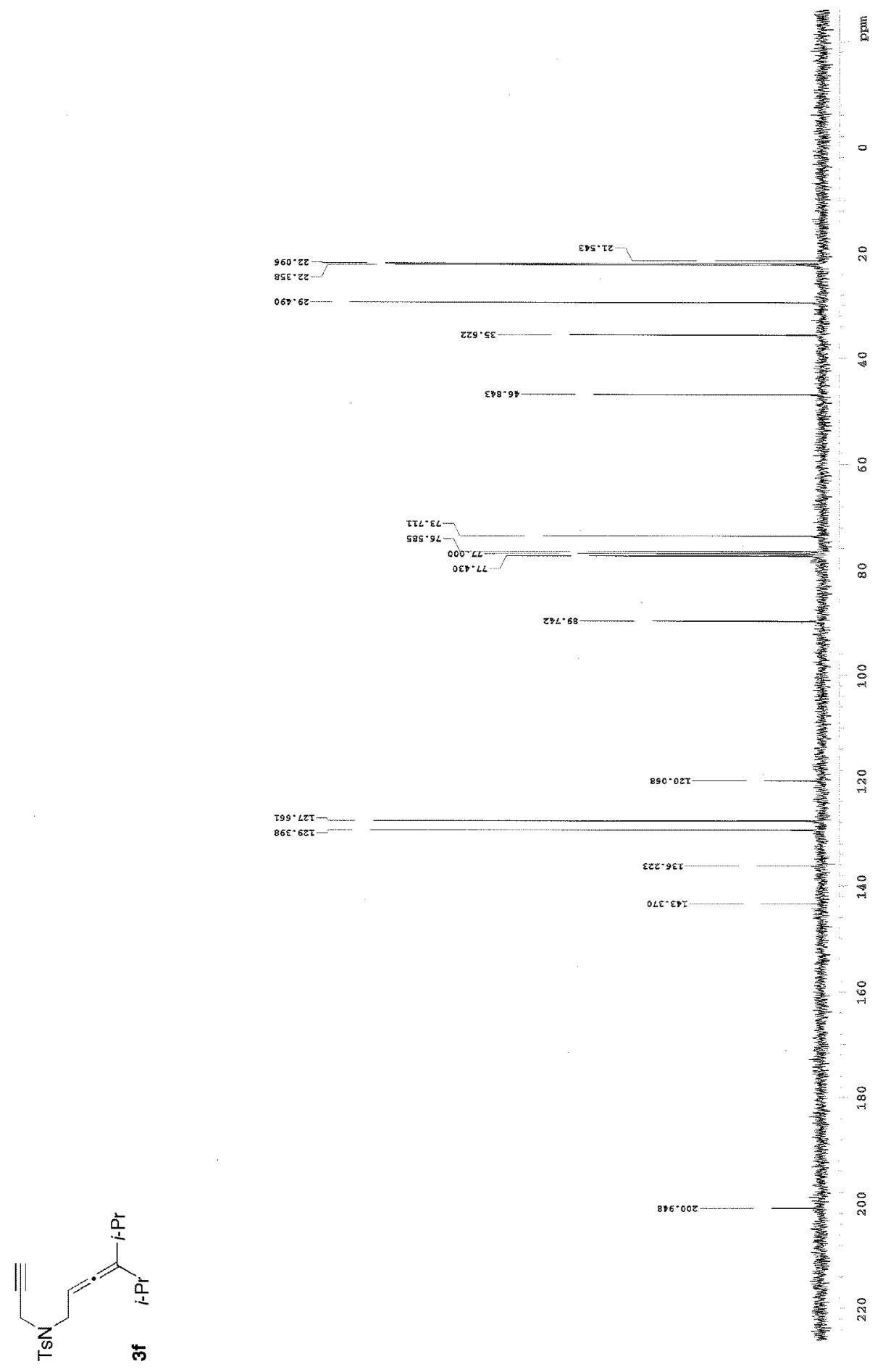




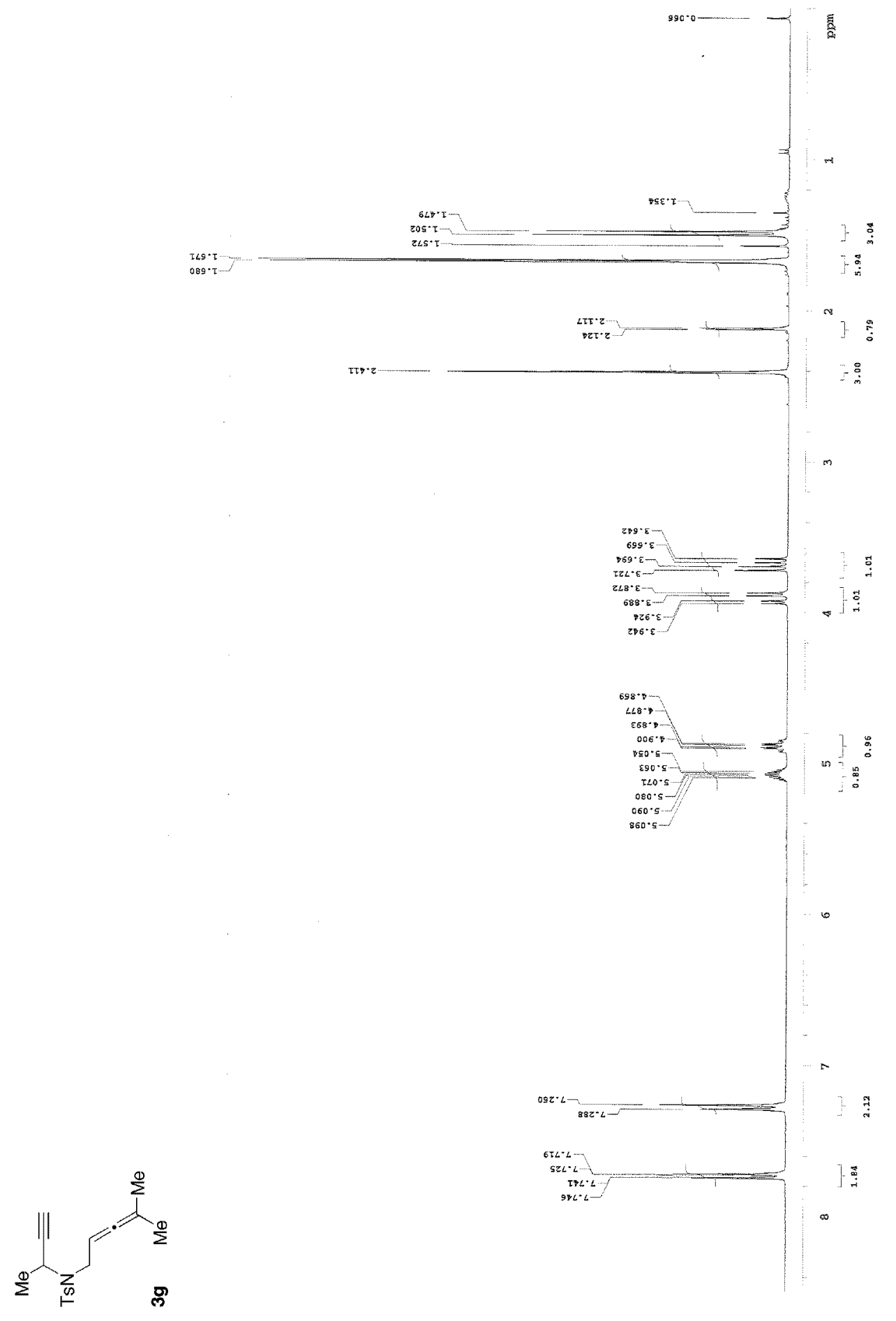




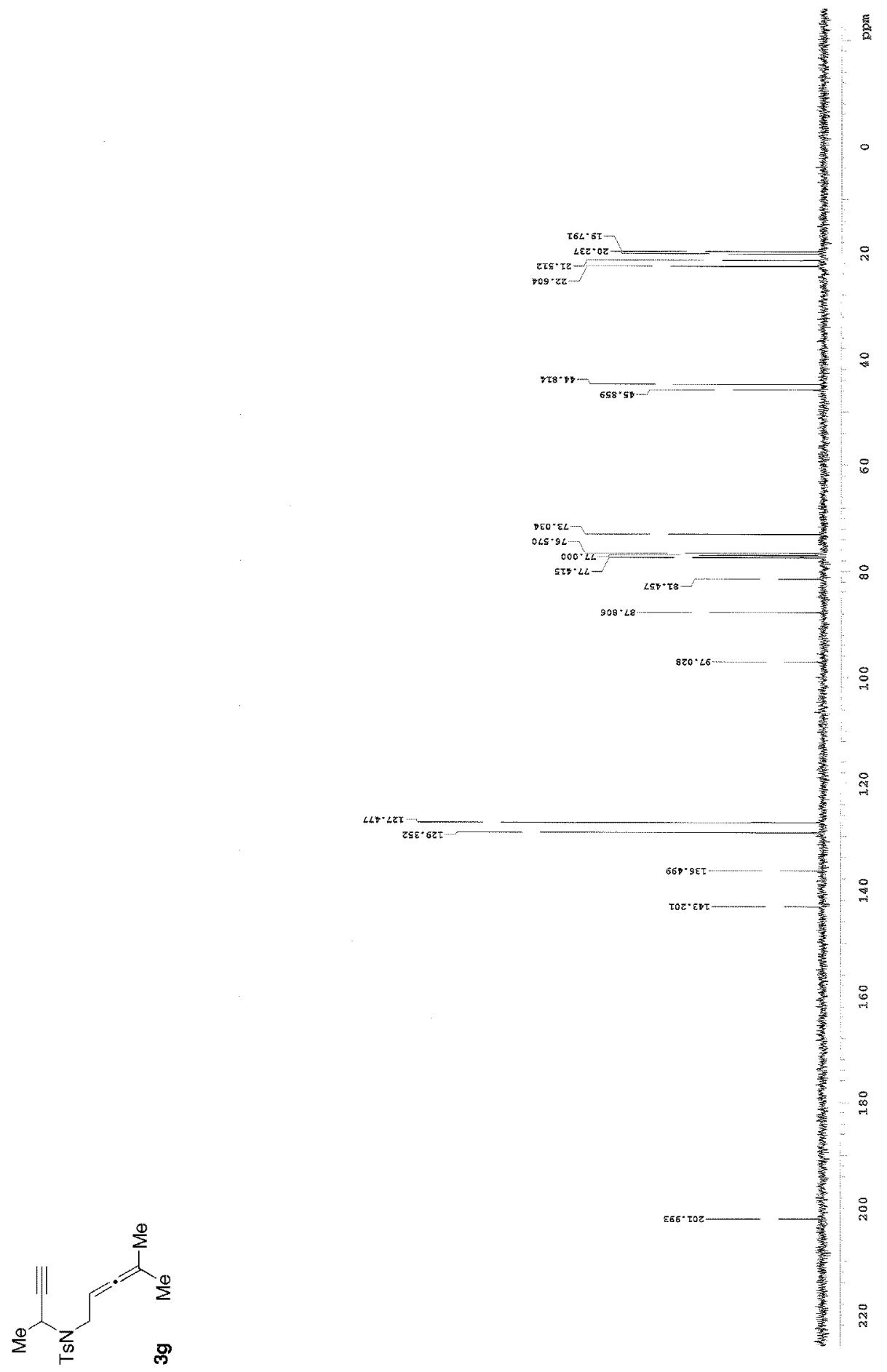




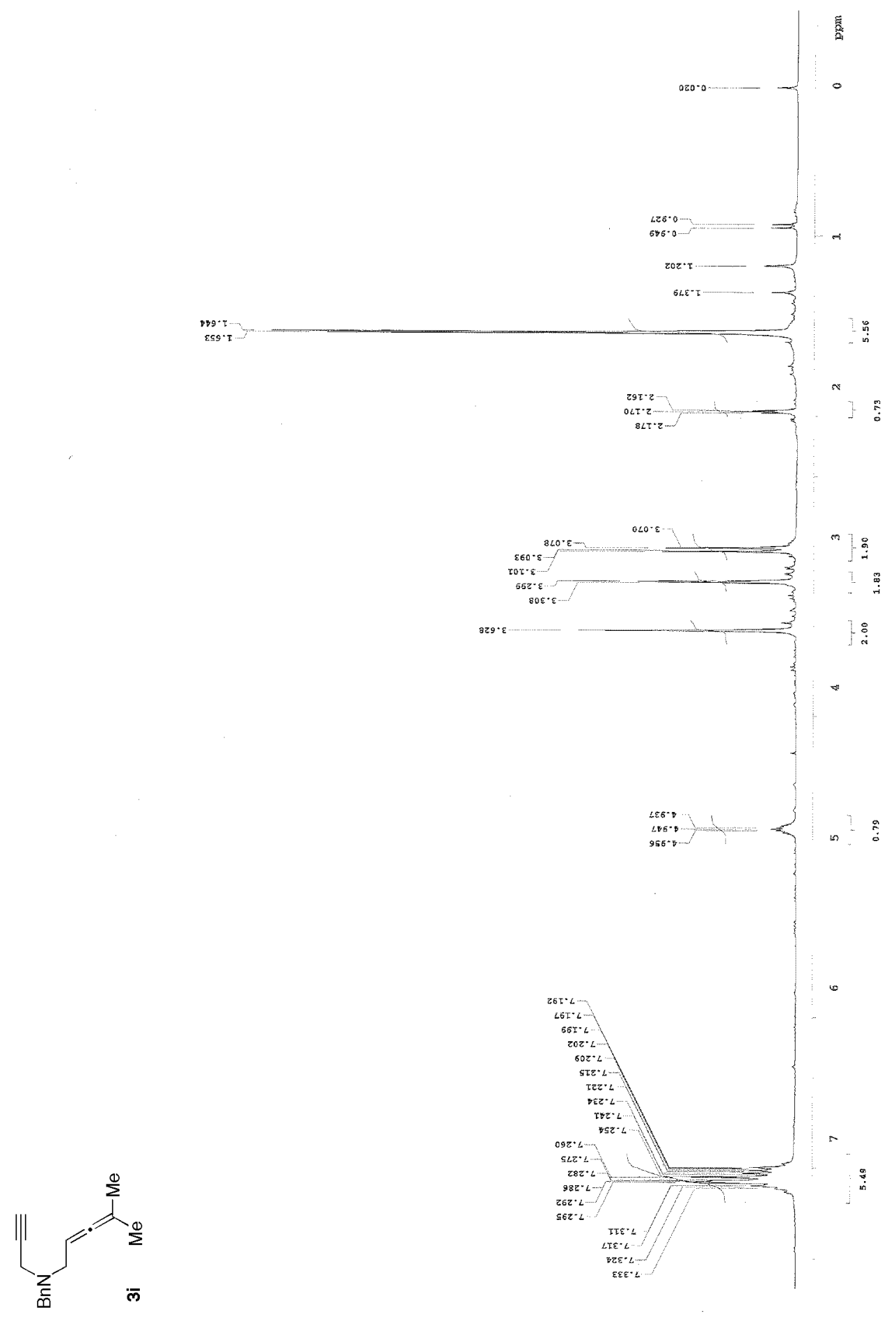




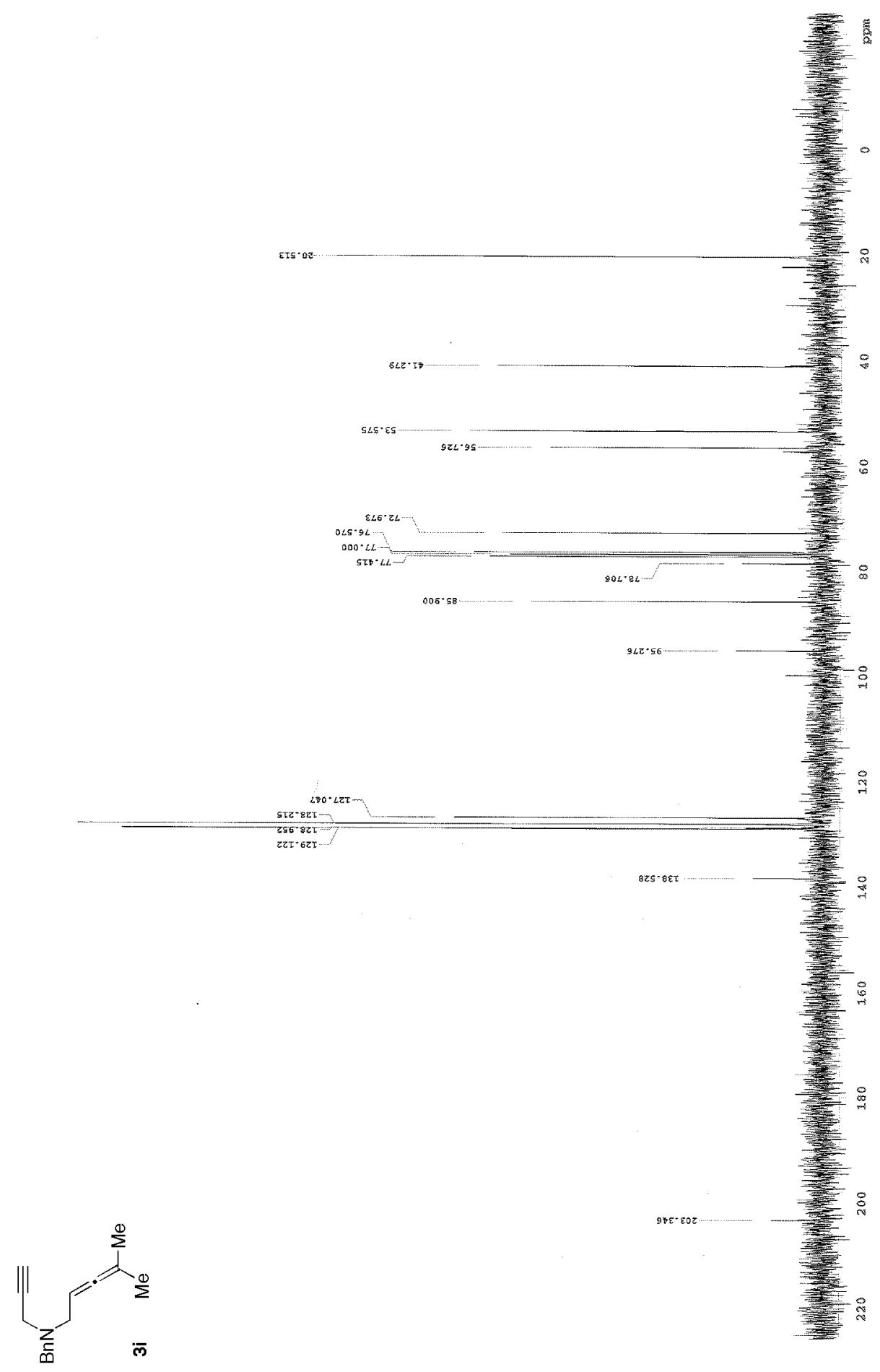




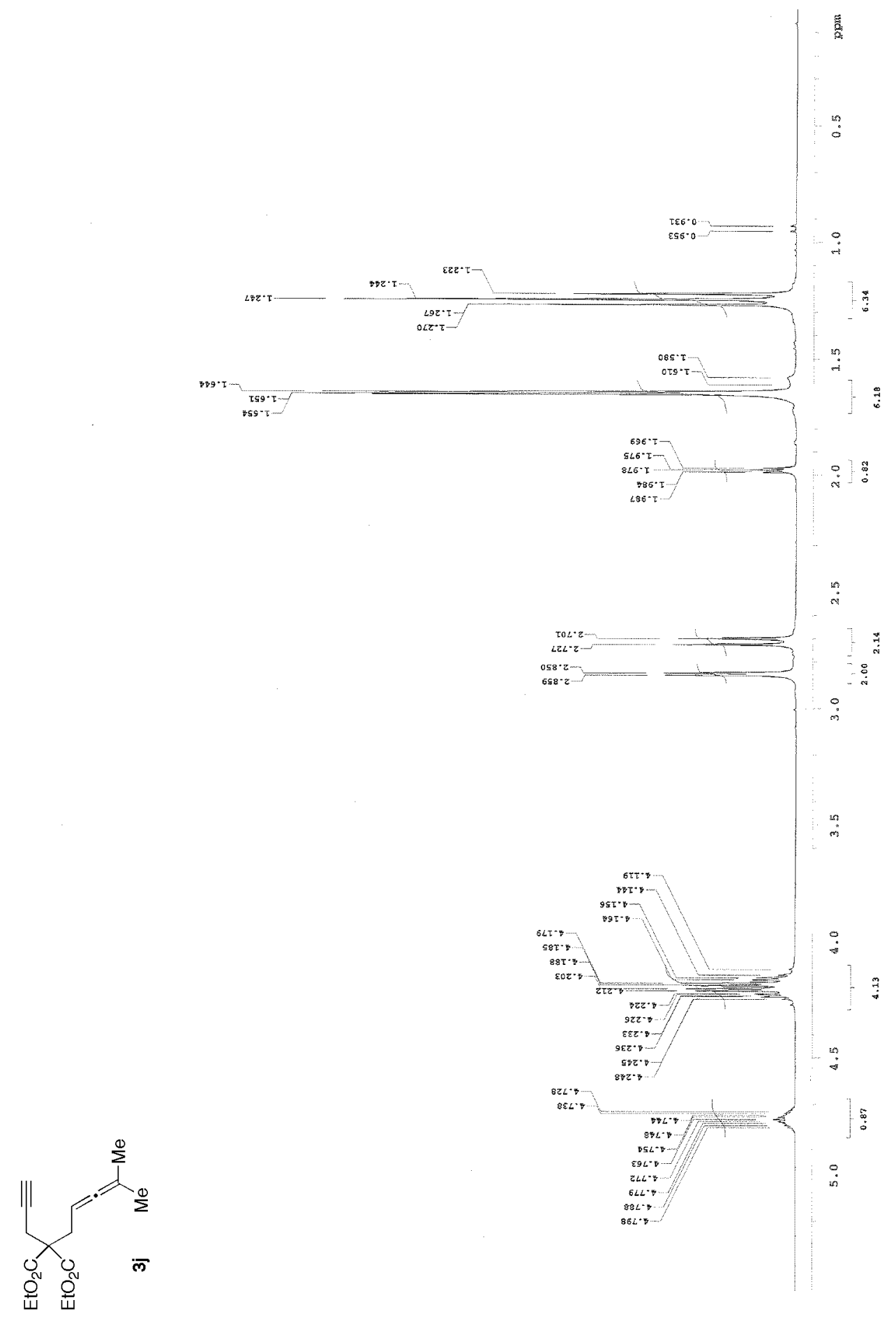




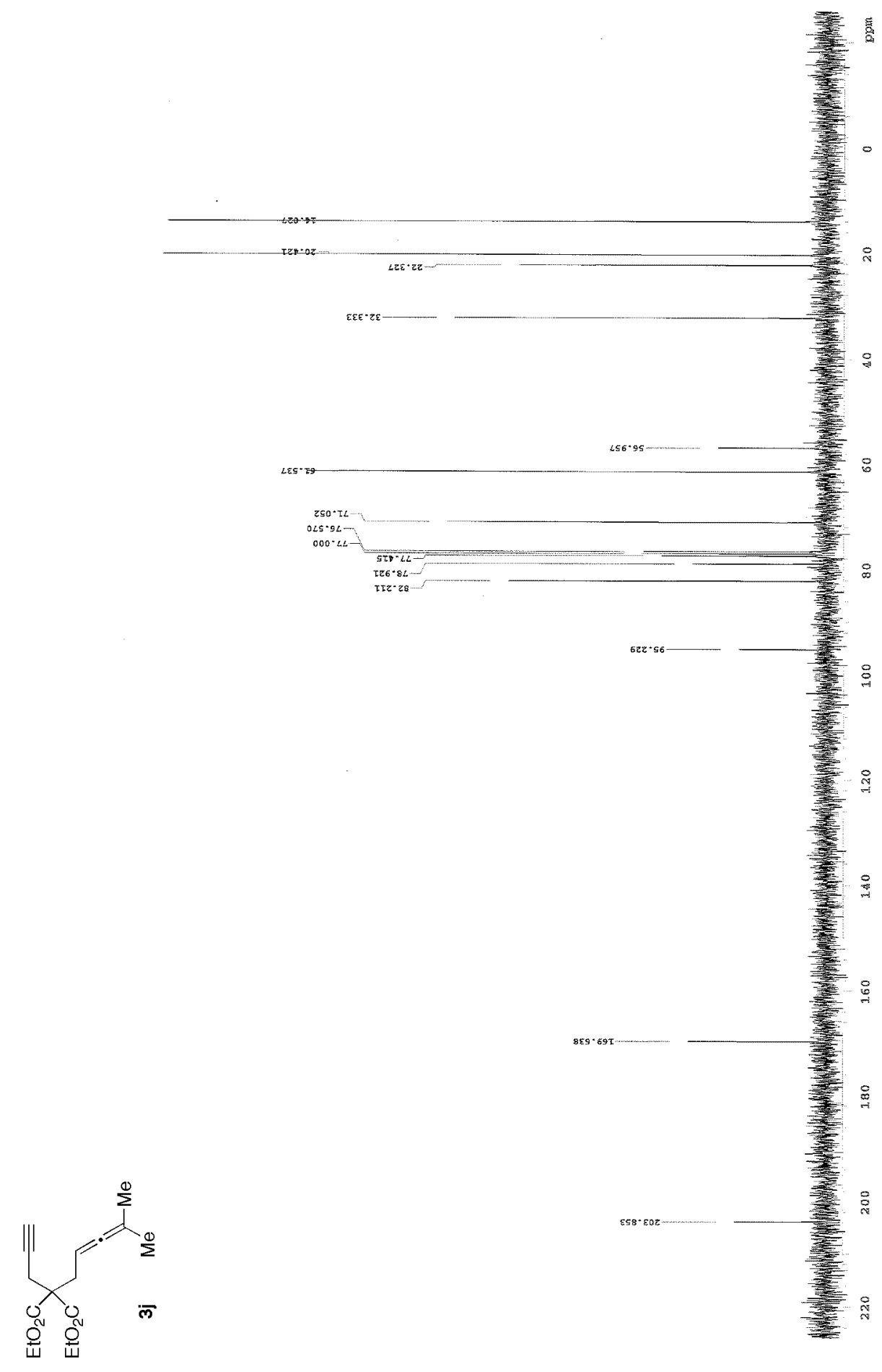









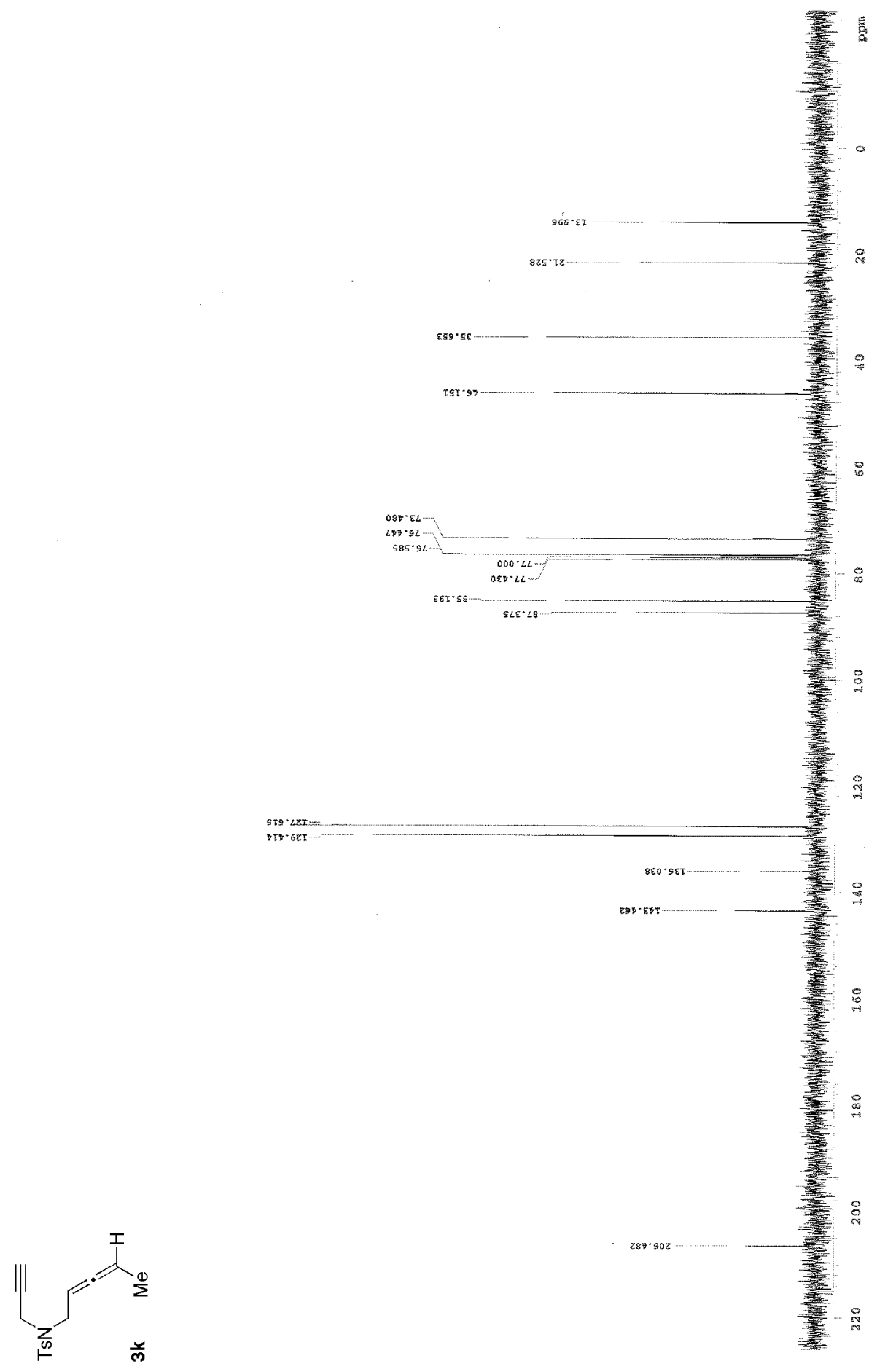




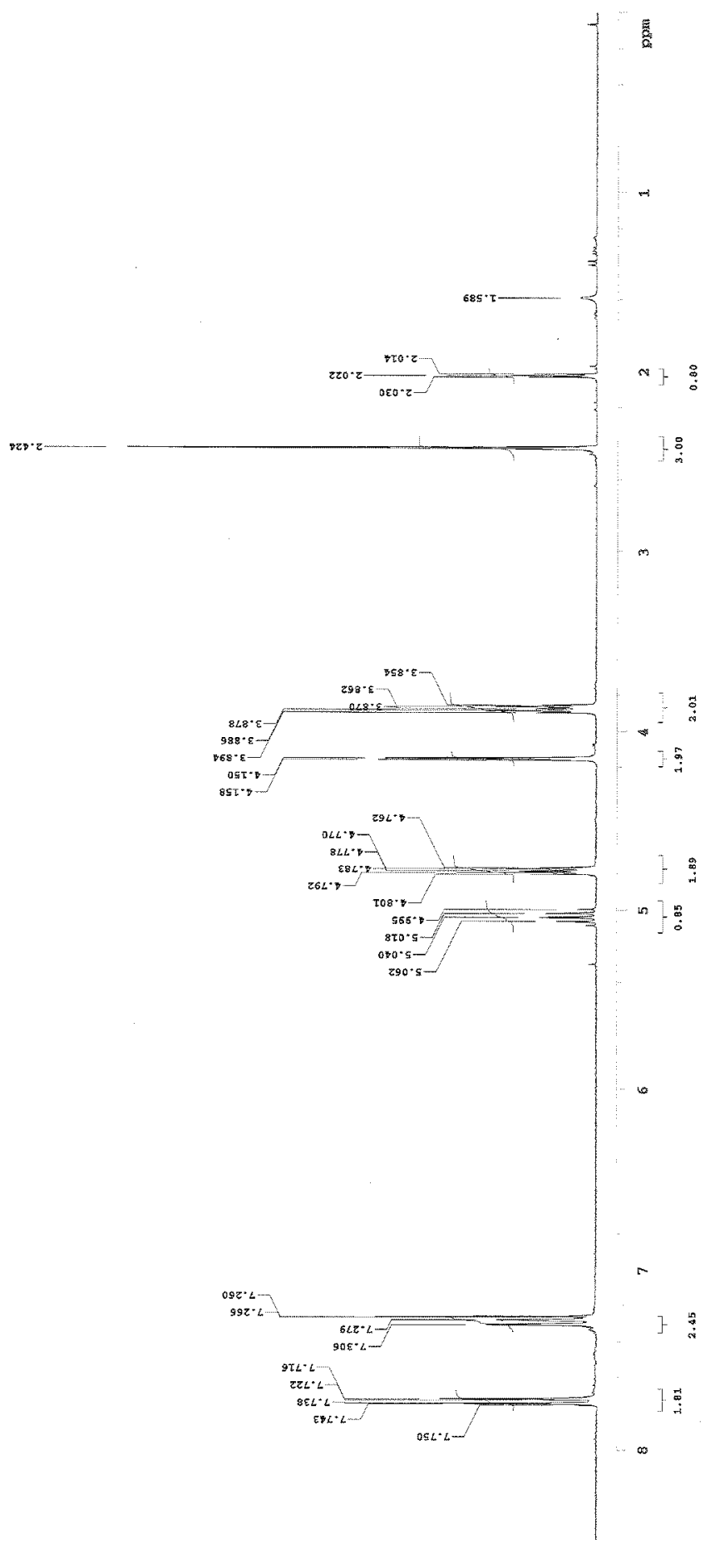




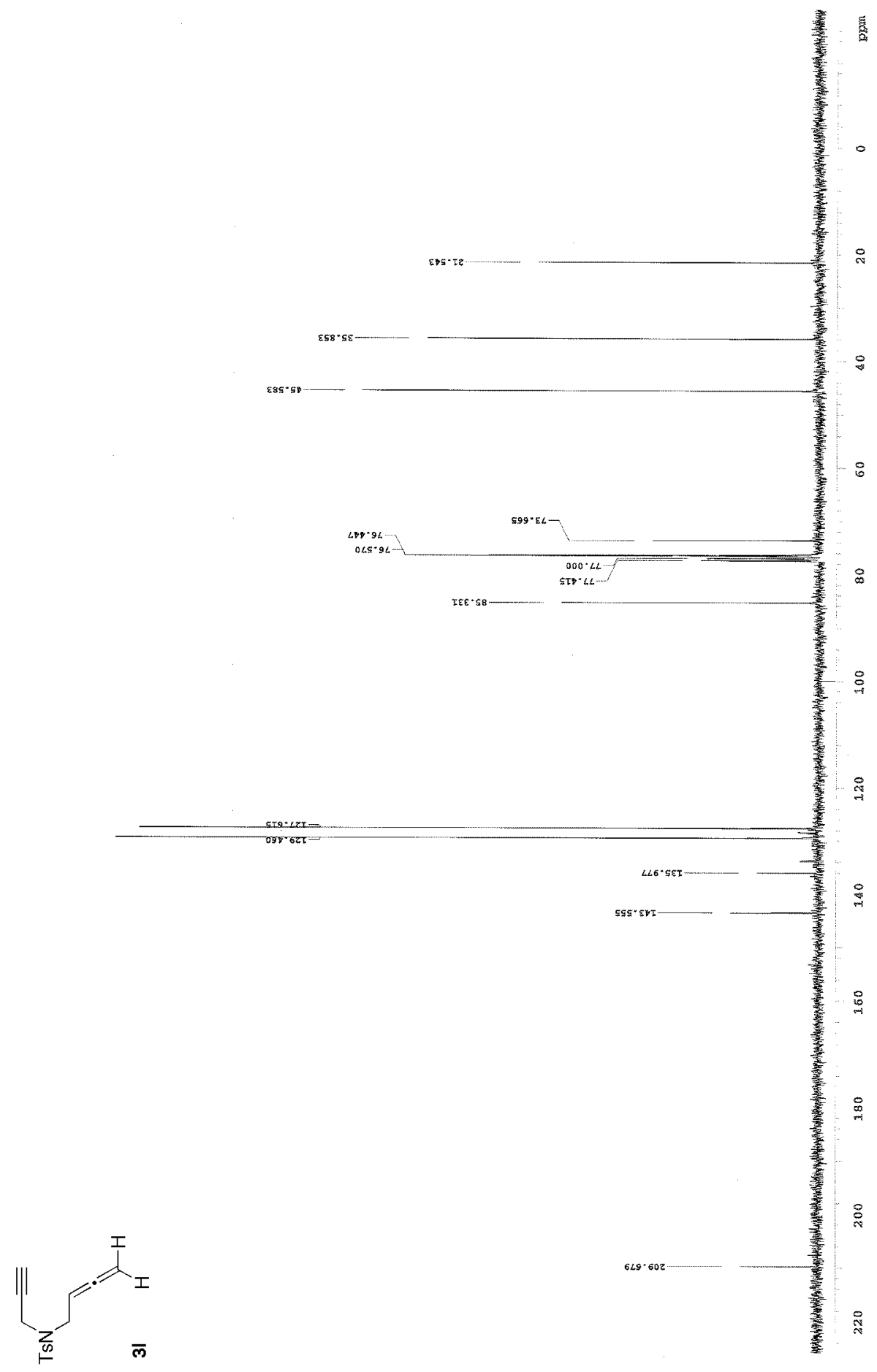




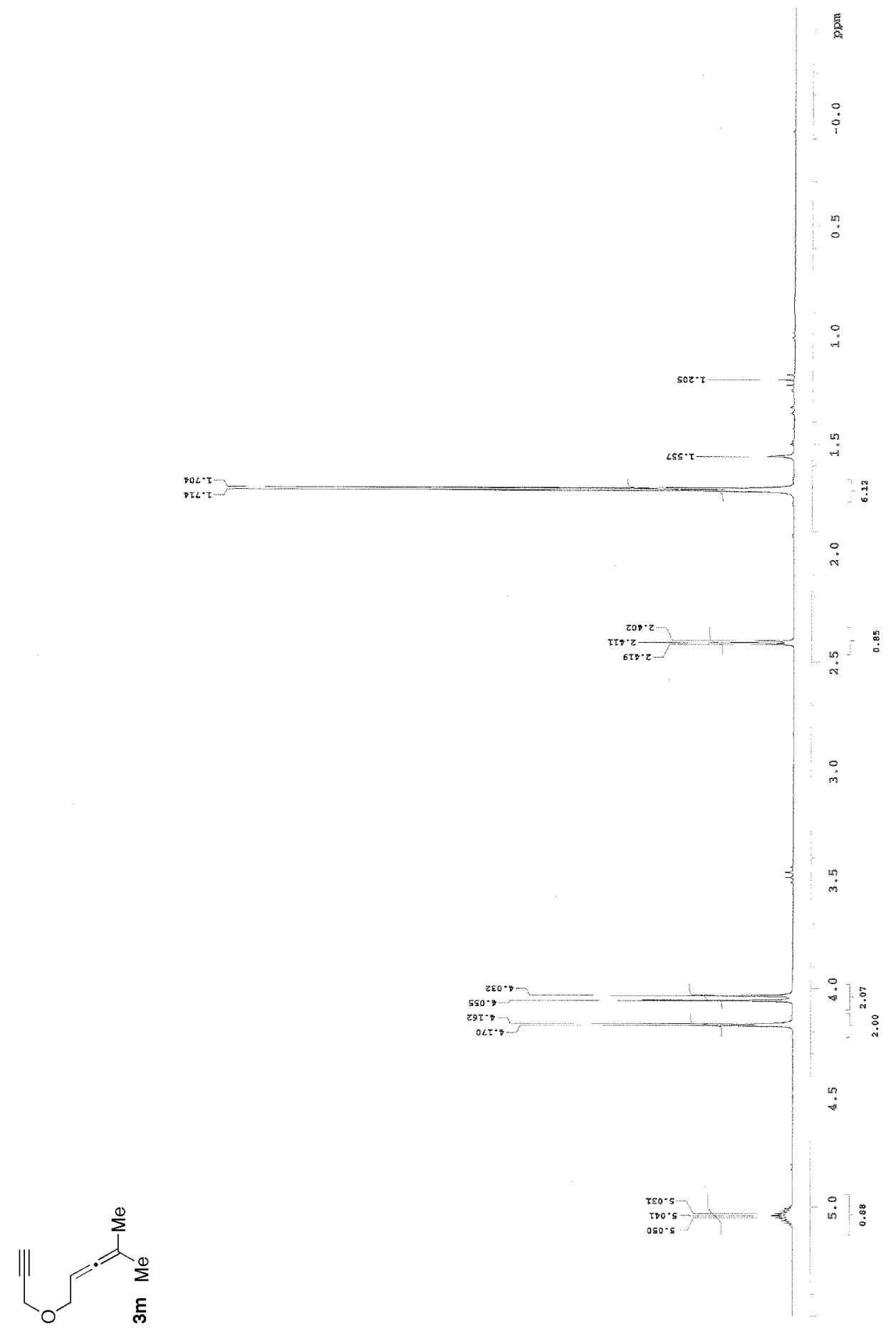




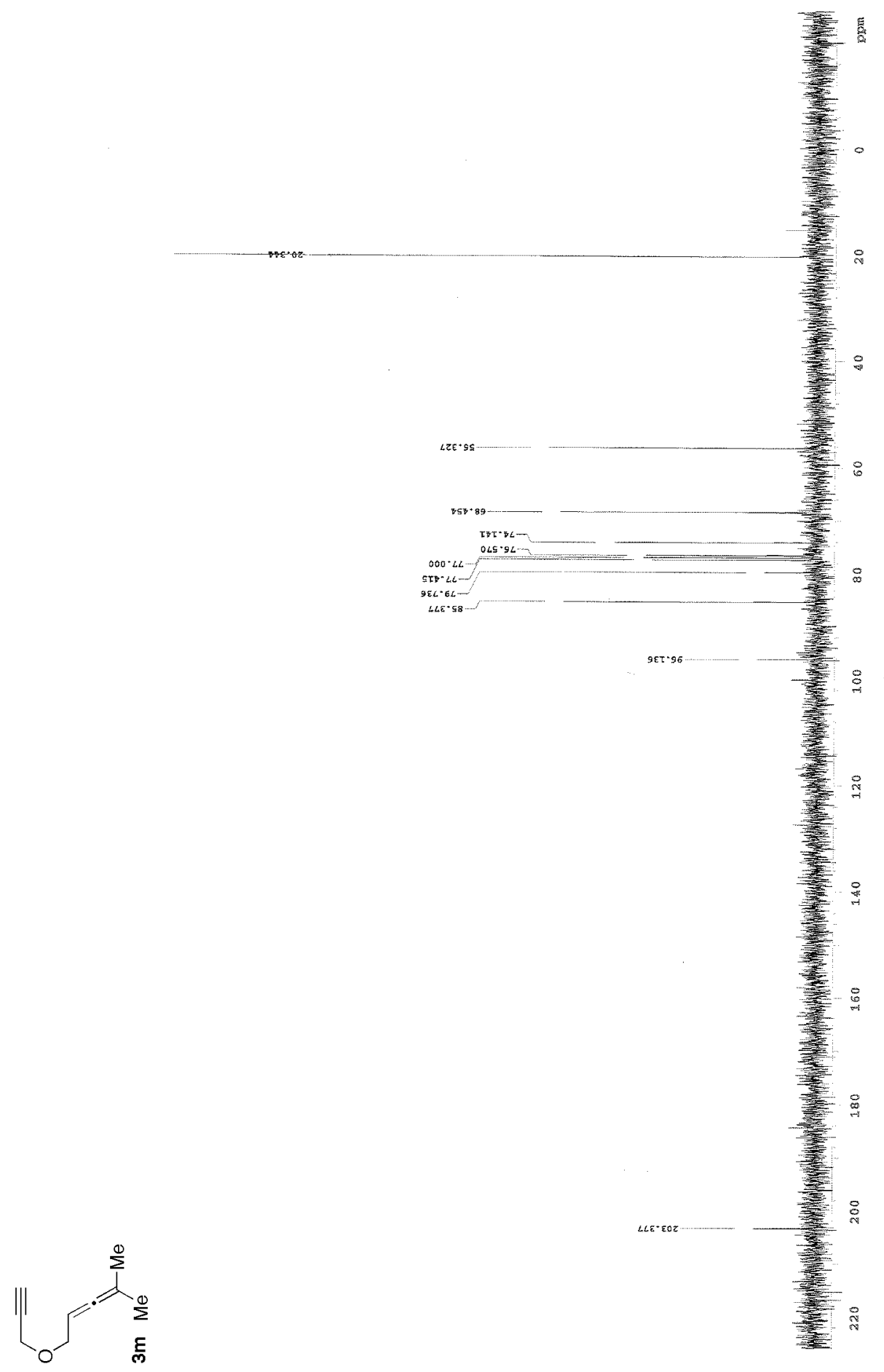




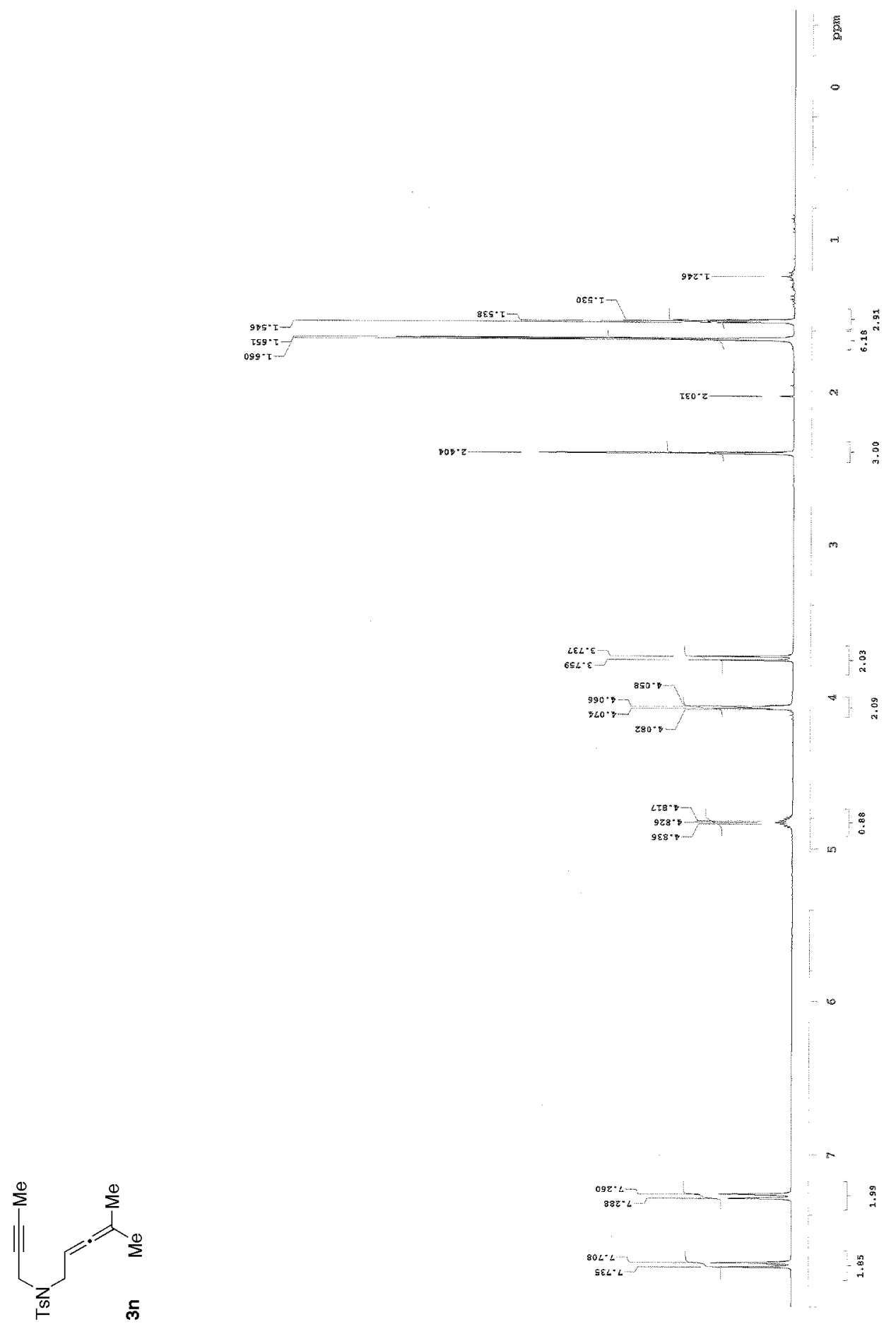




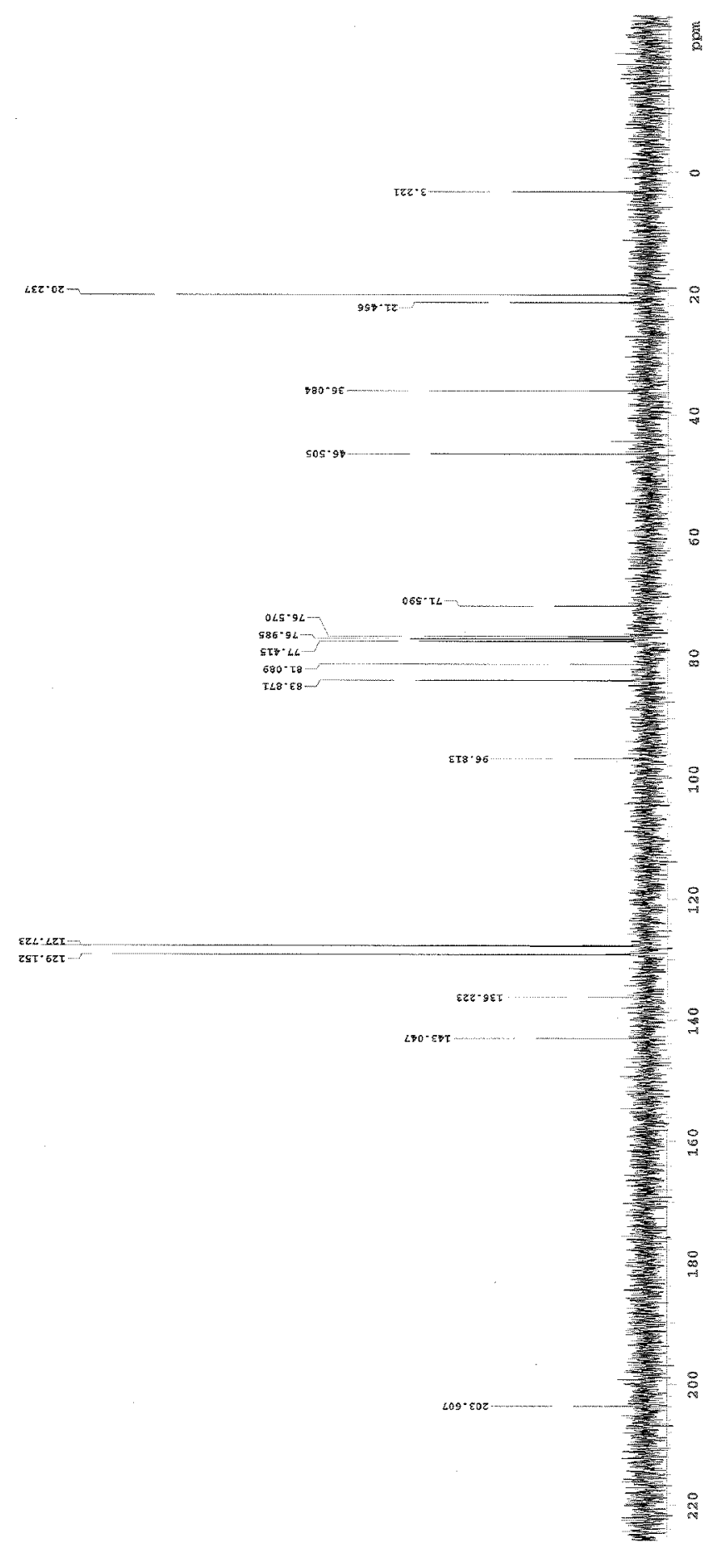




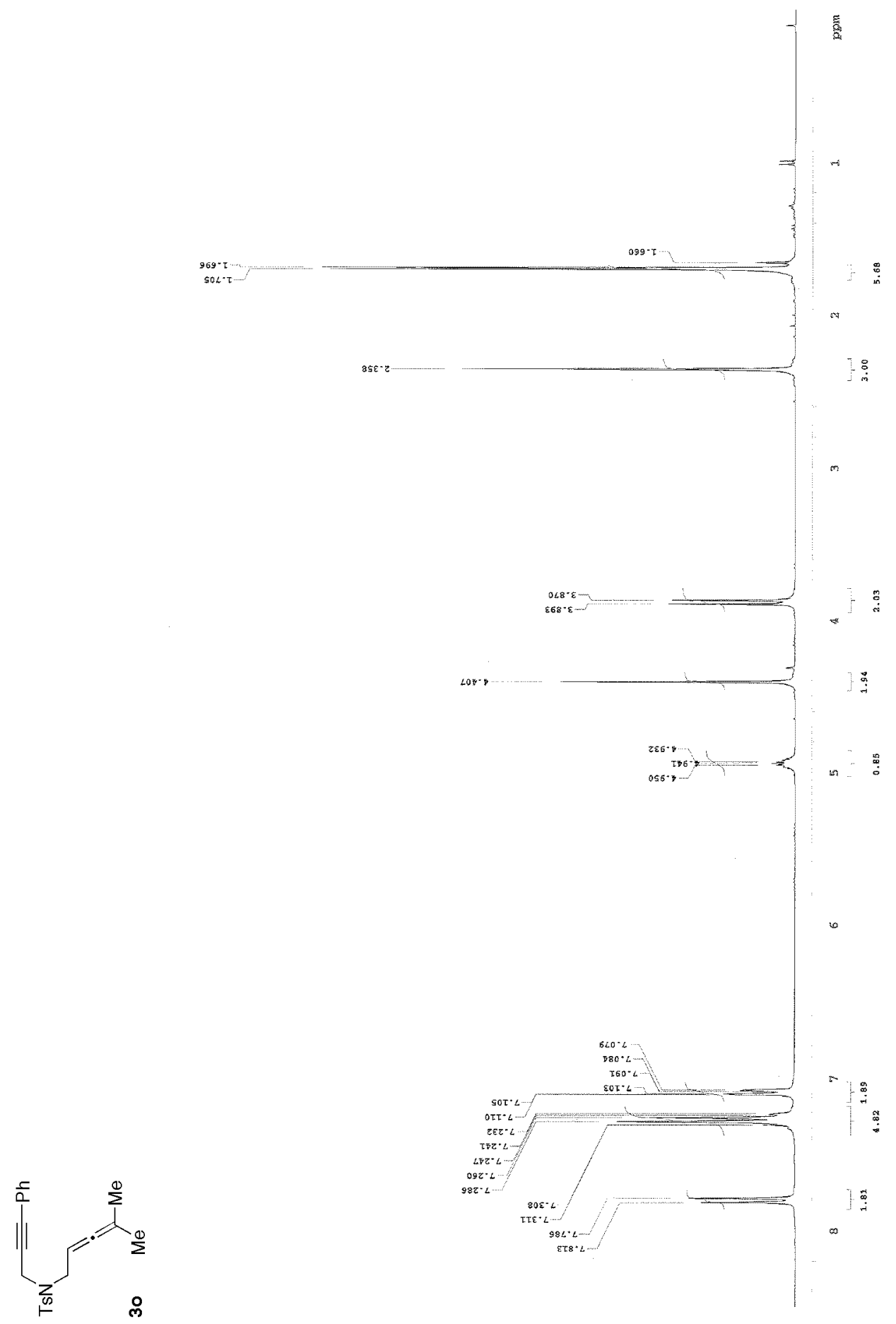




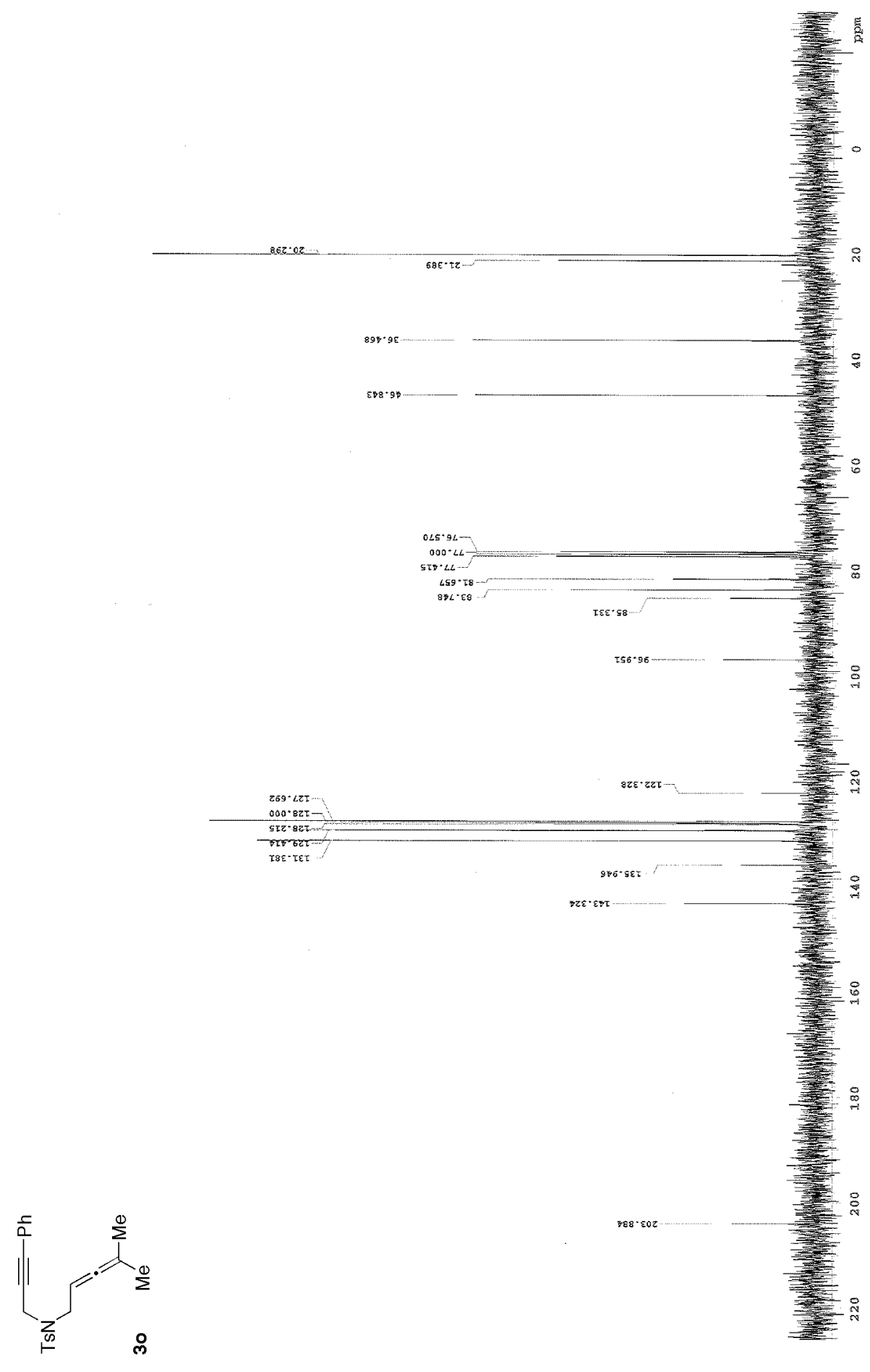




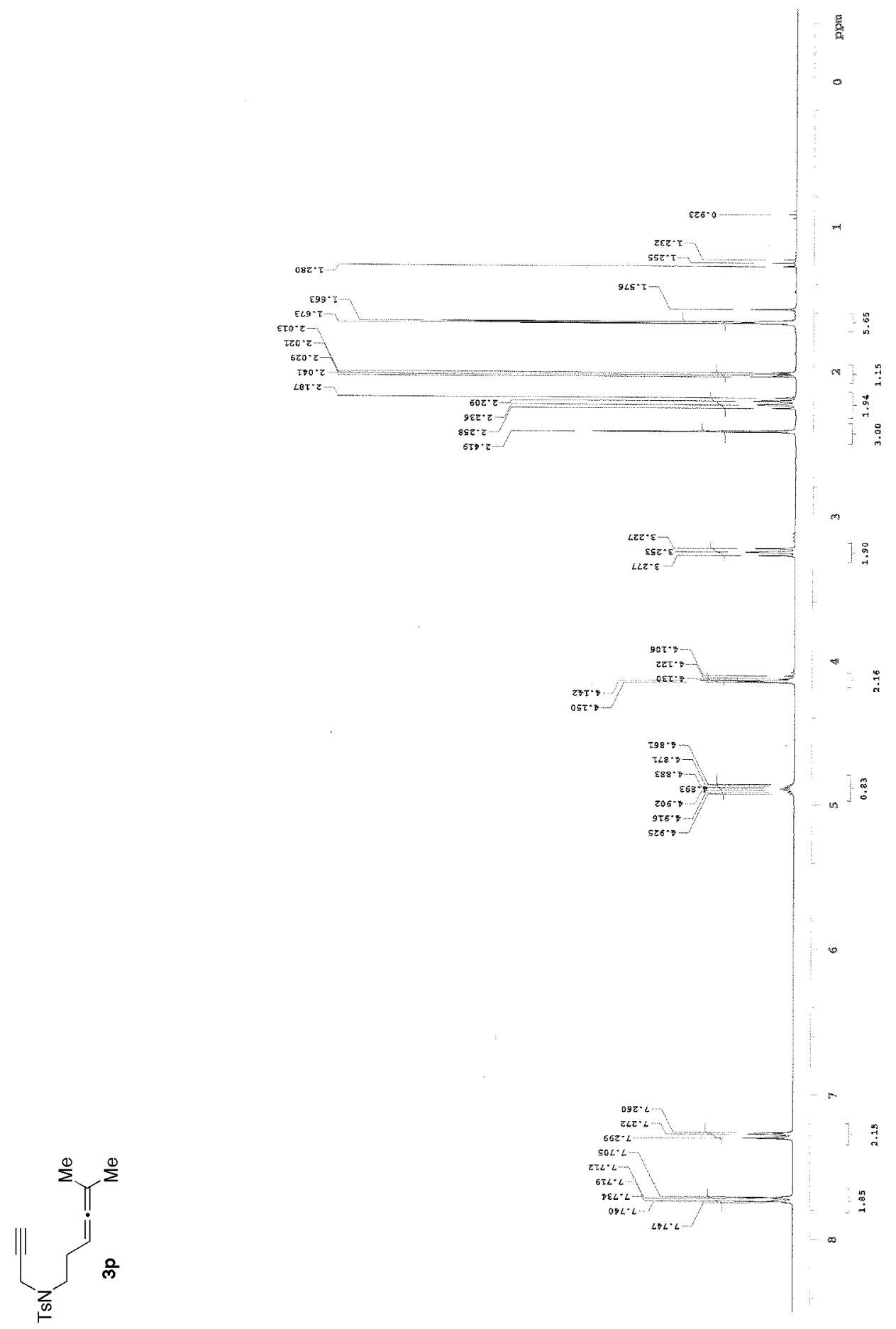




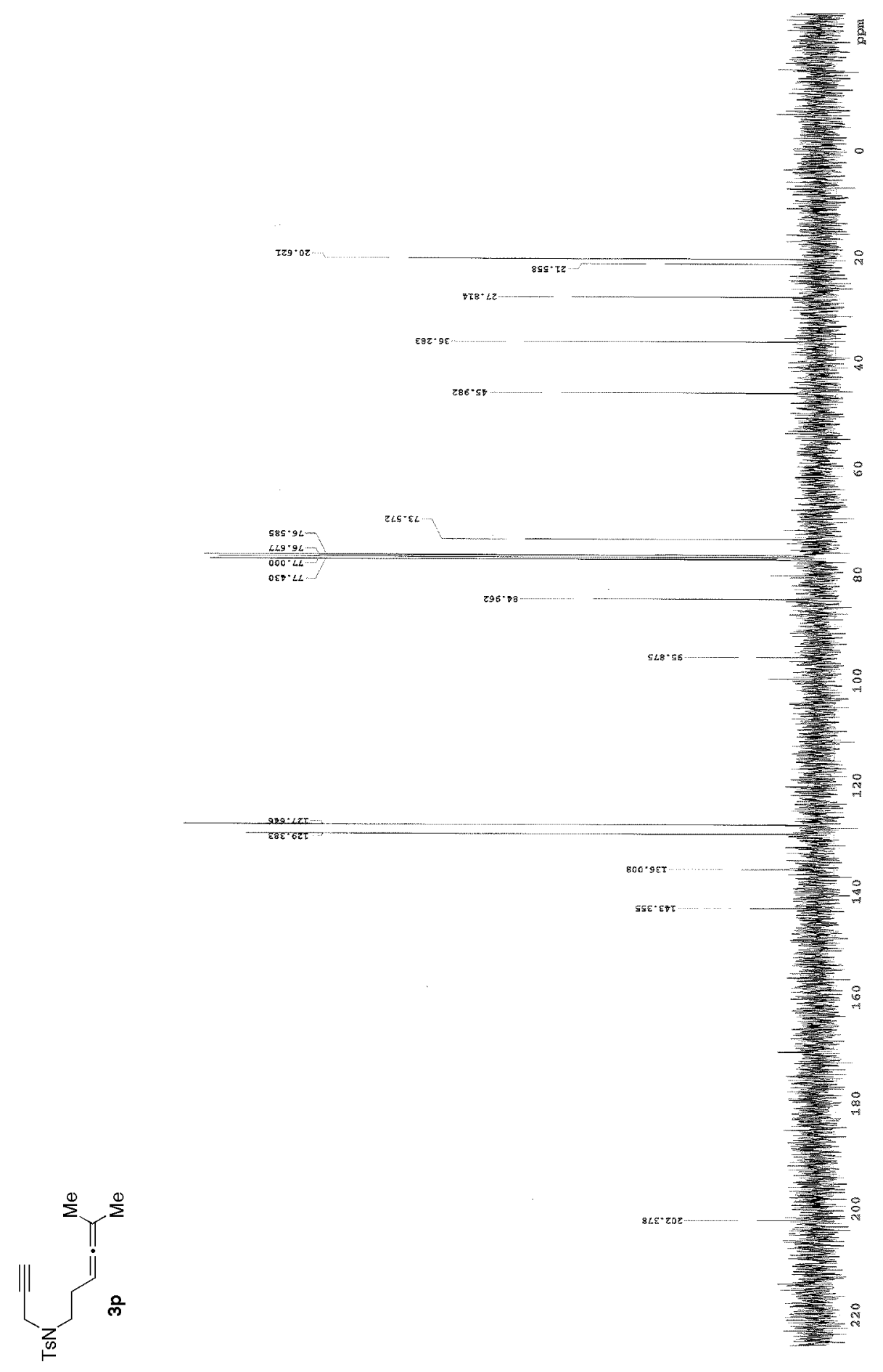




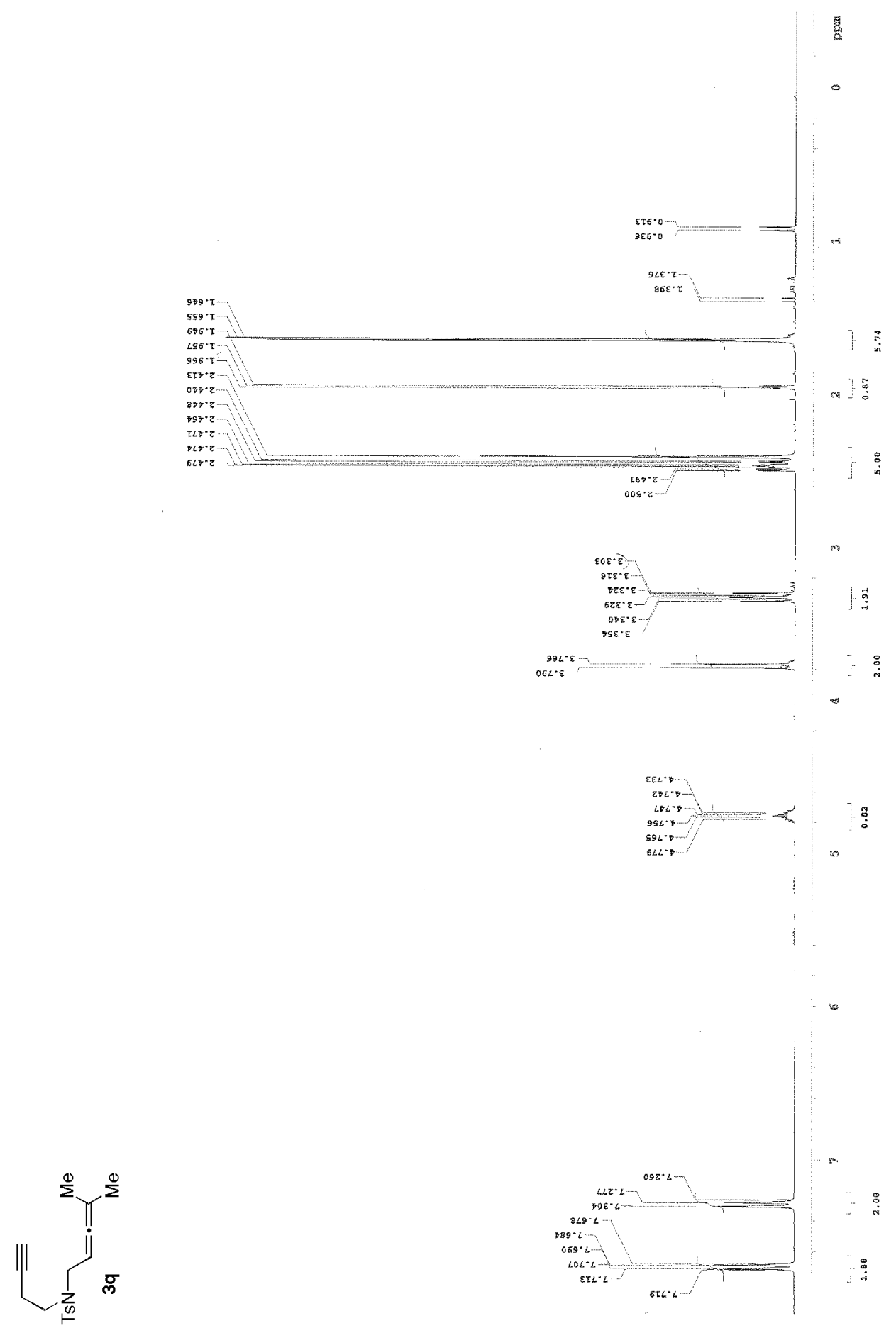




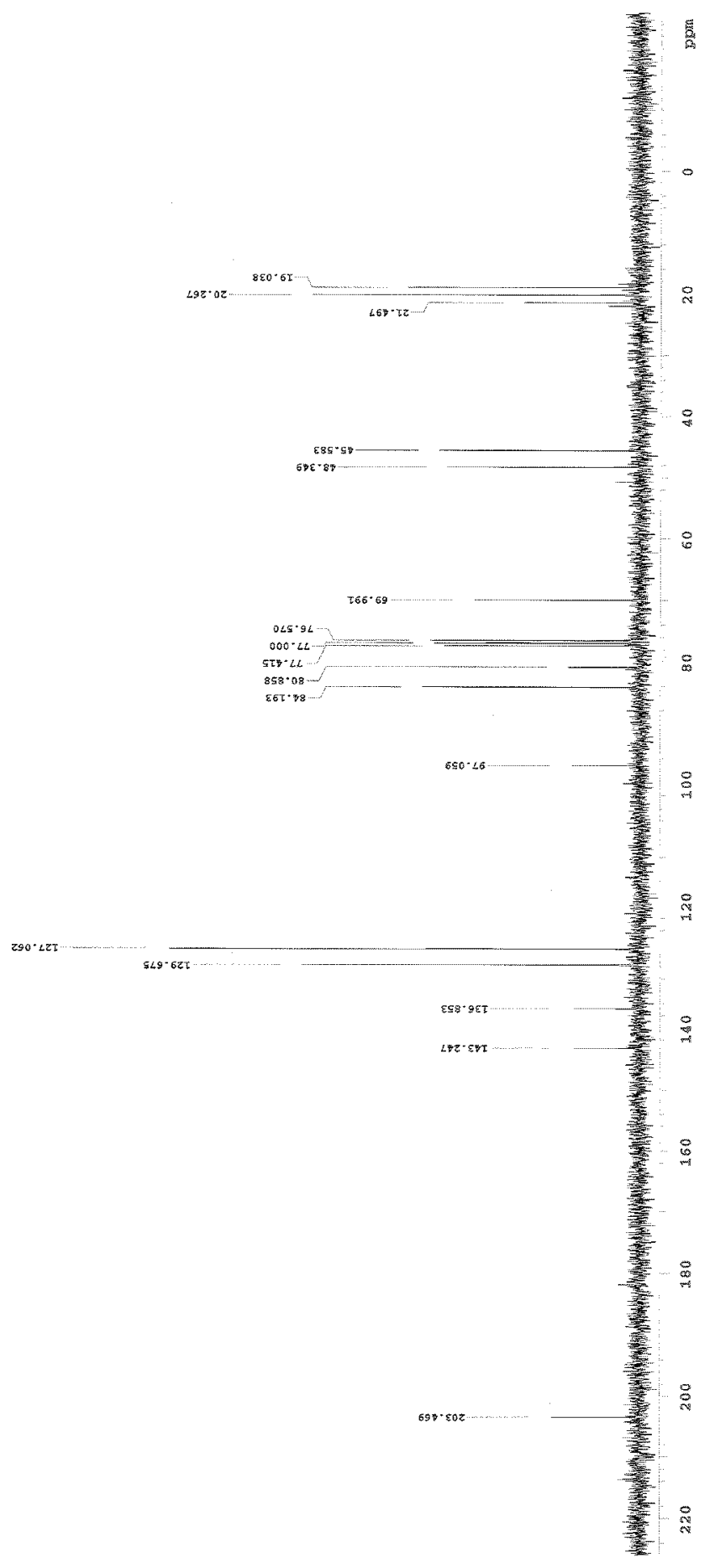




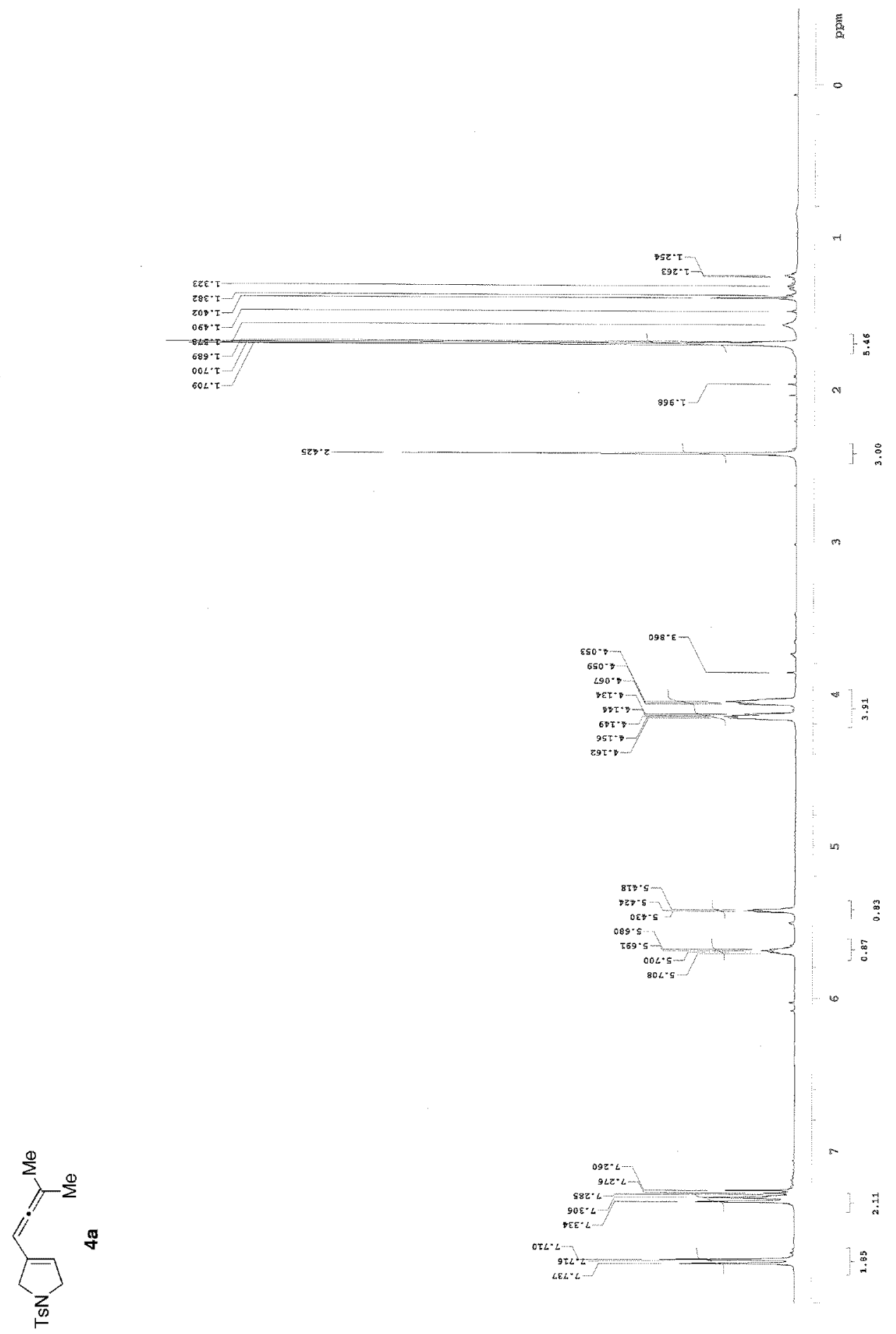




$$
1
$$




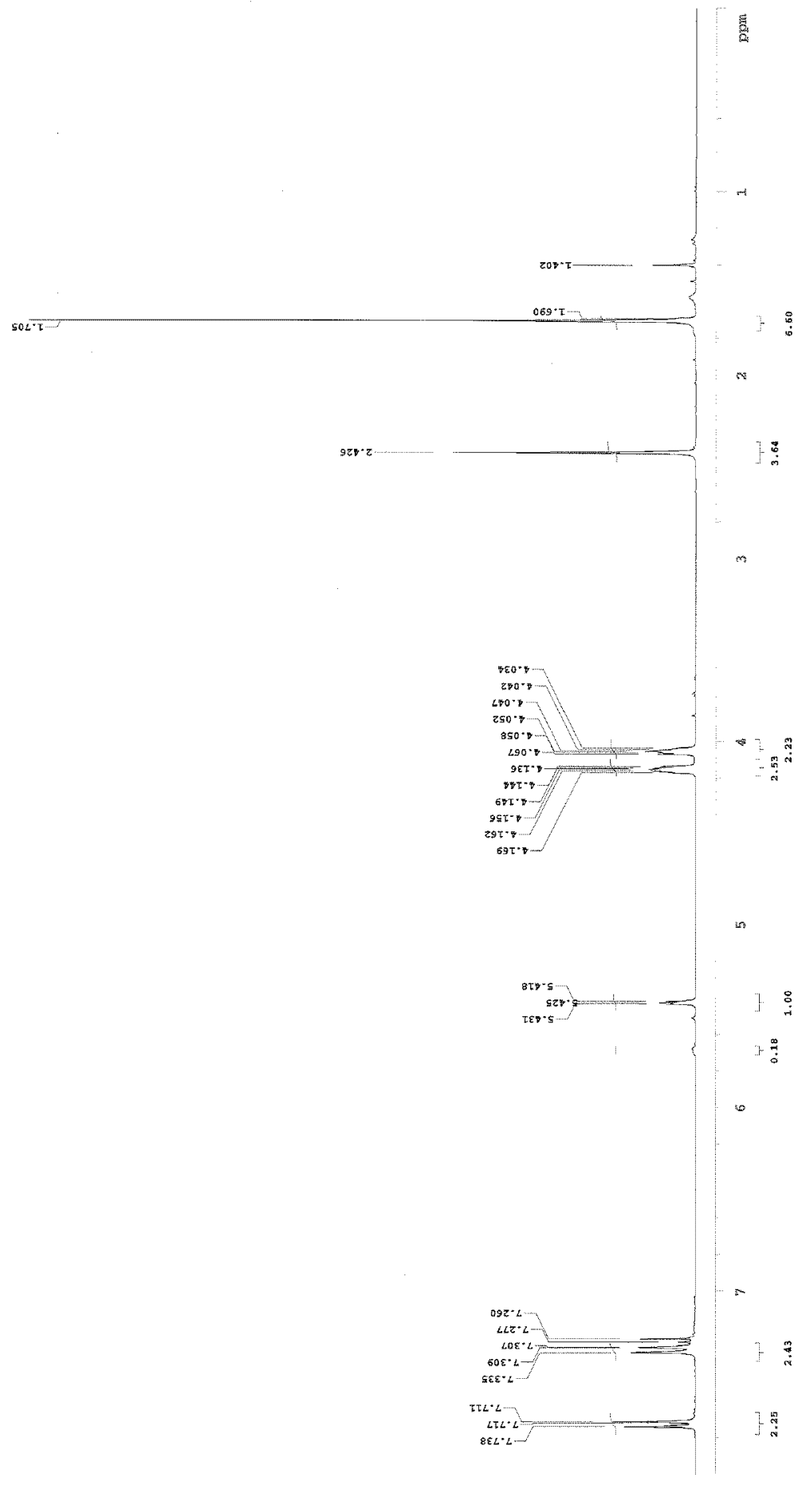




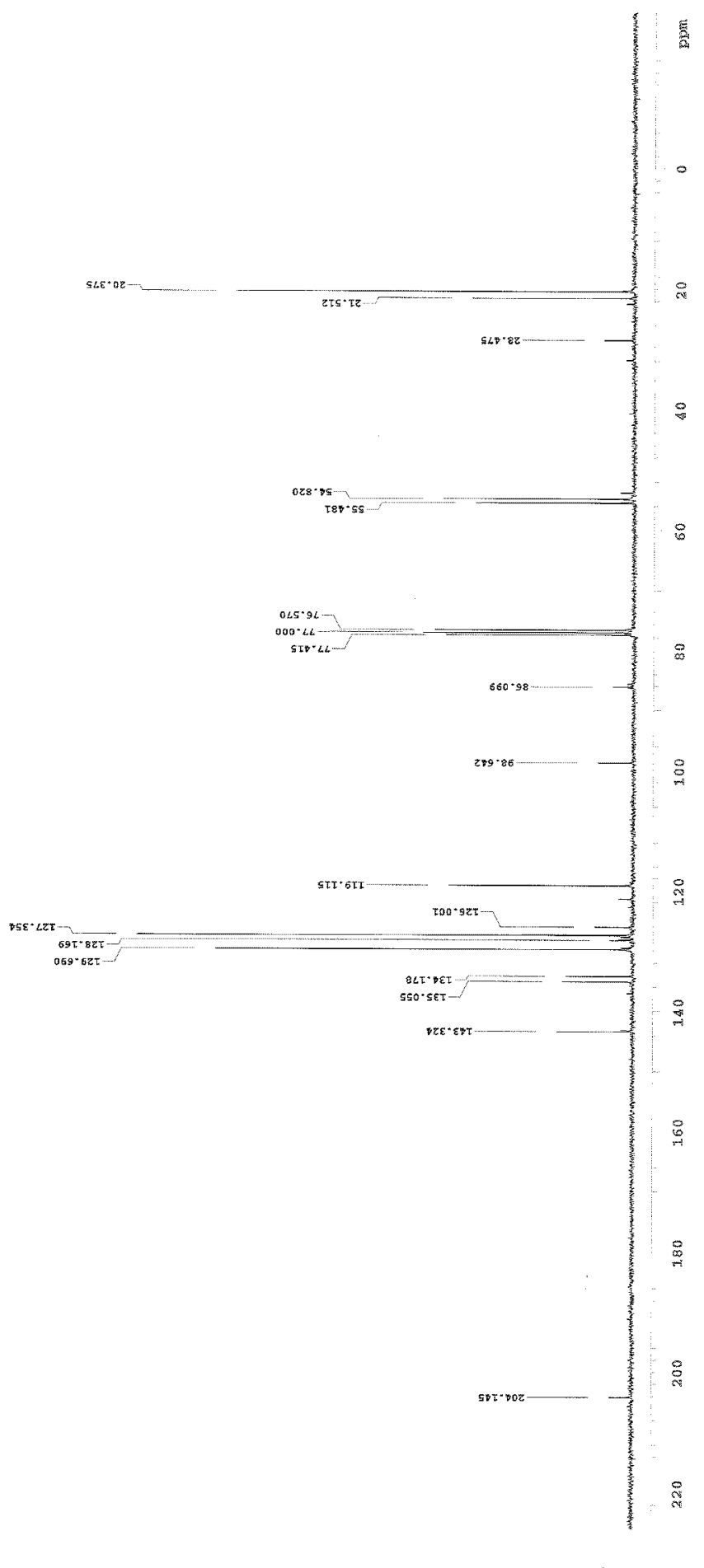




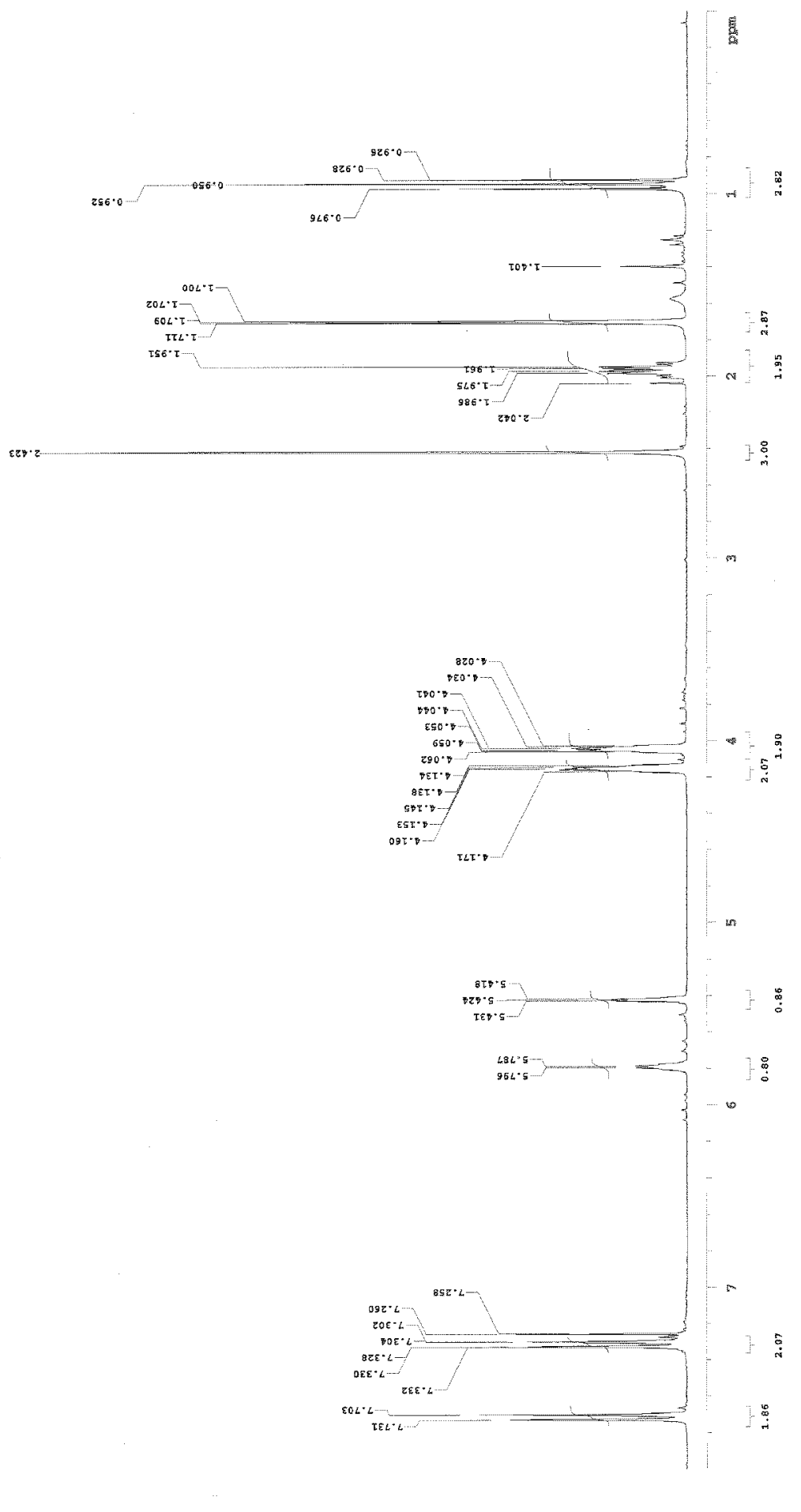



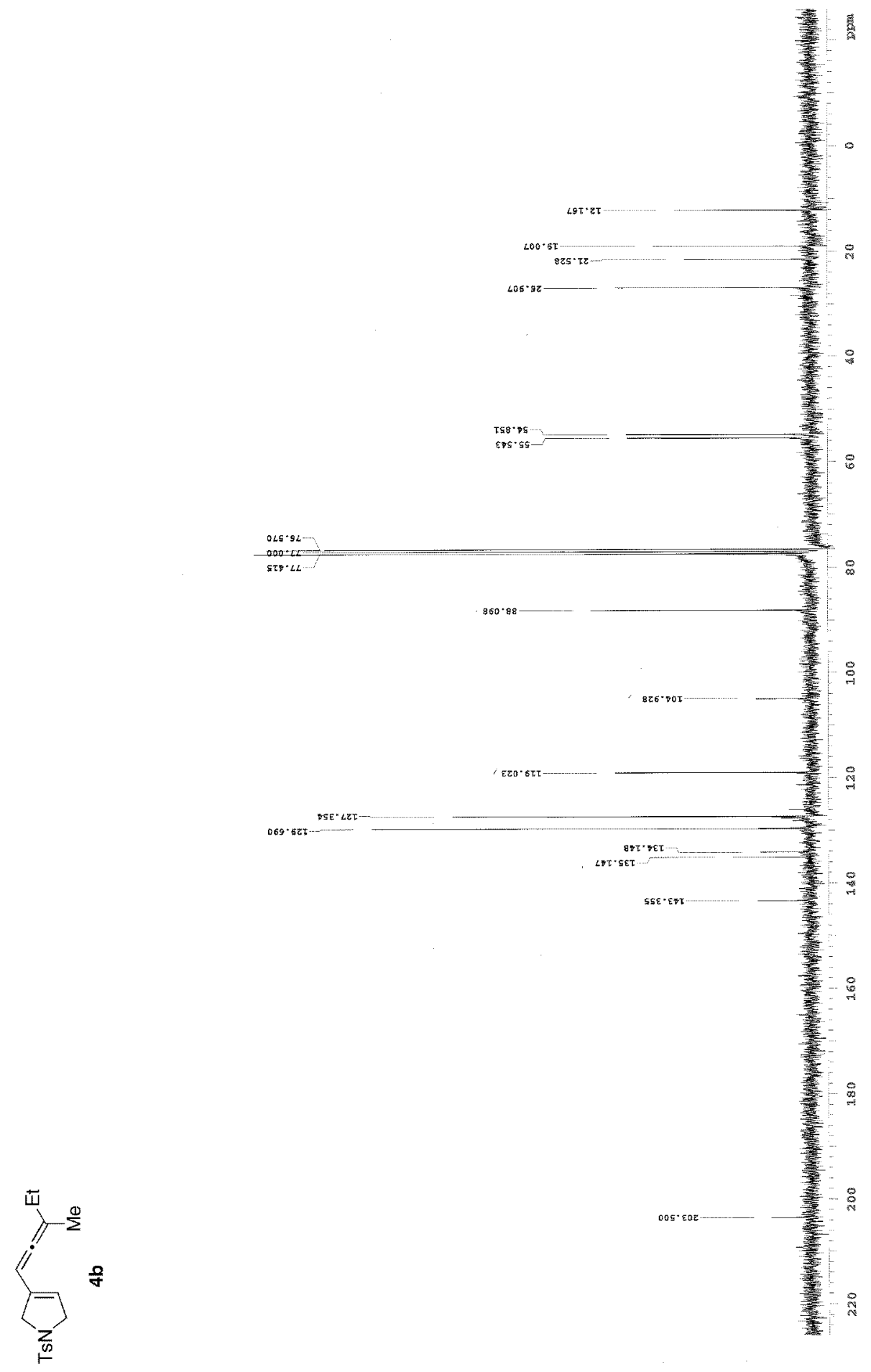

S51 


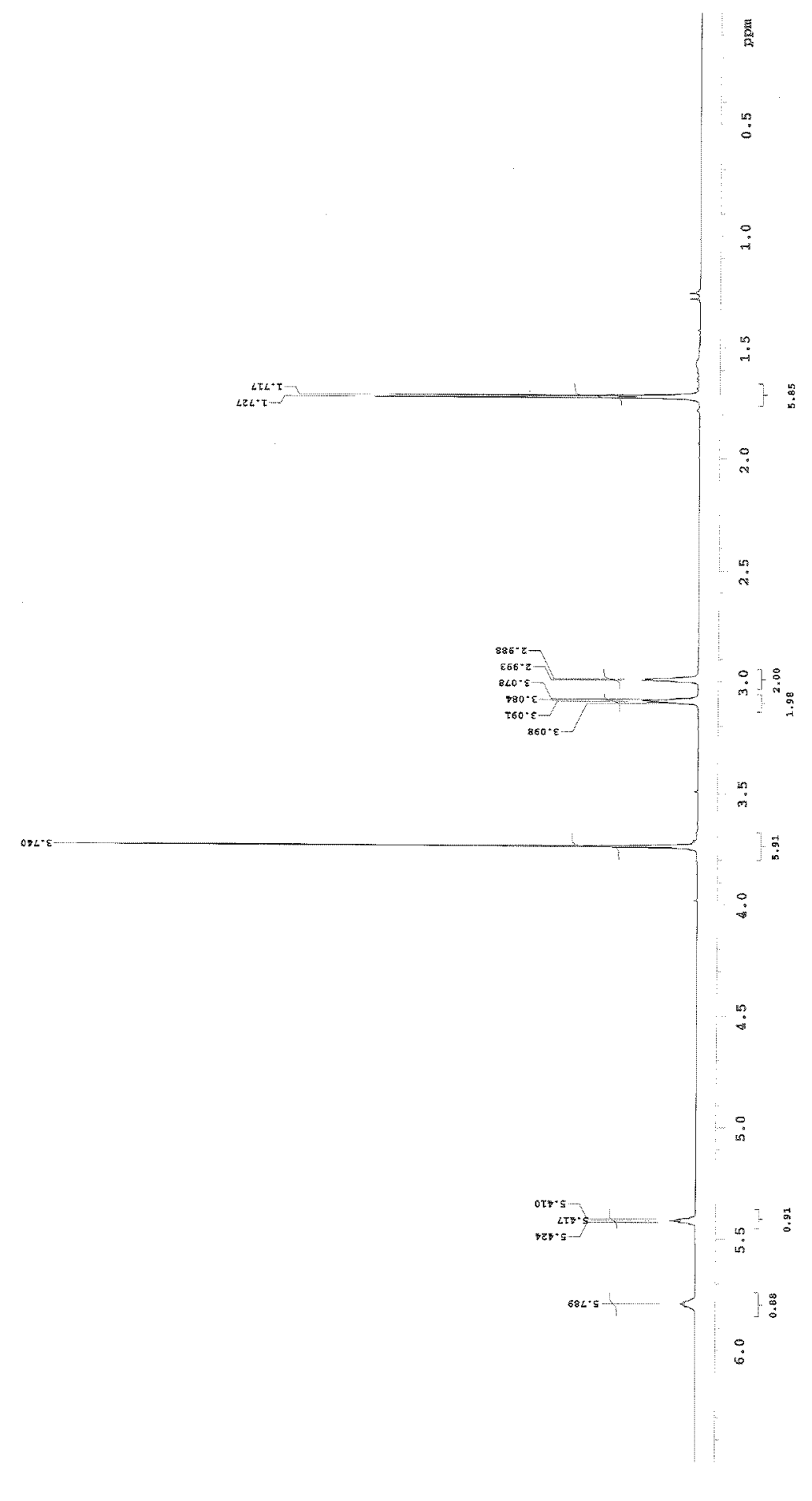




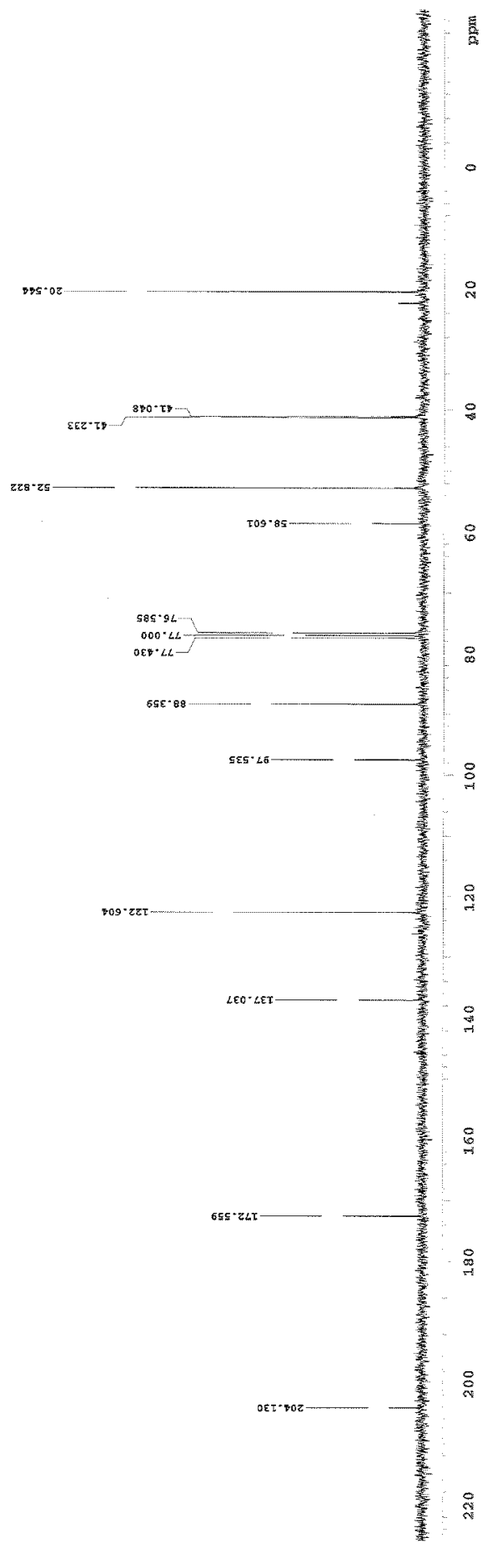




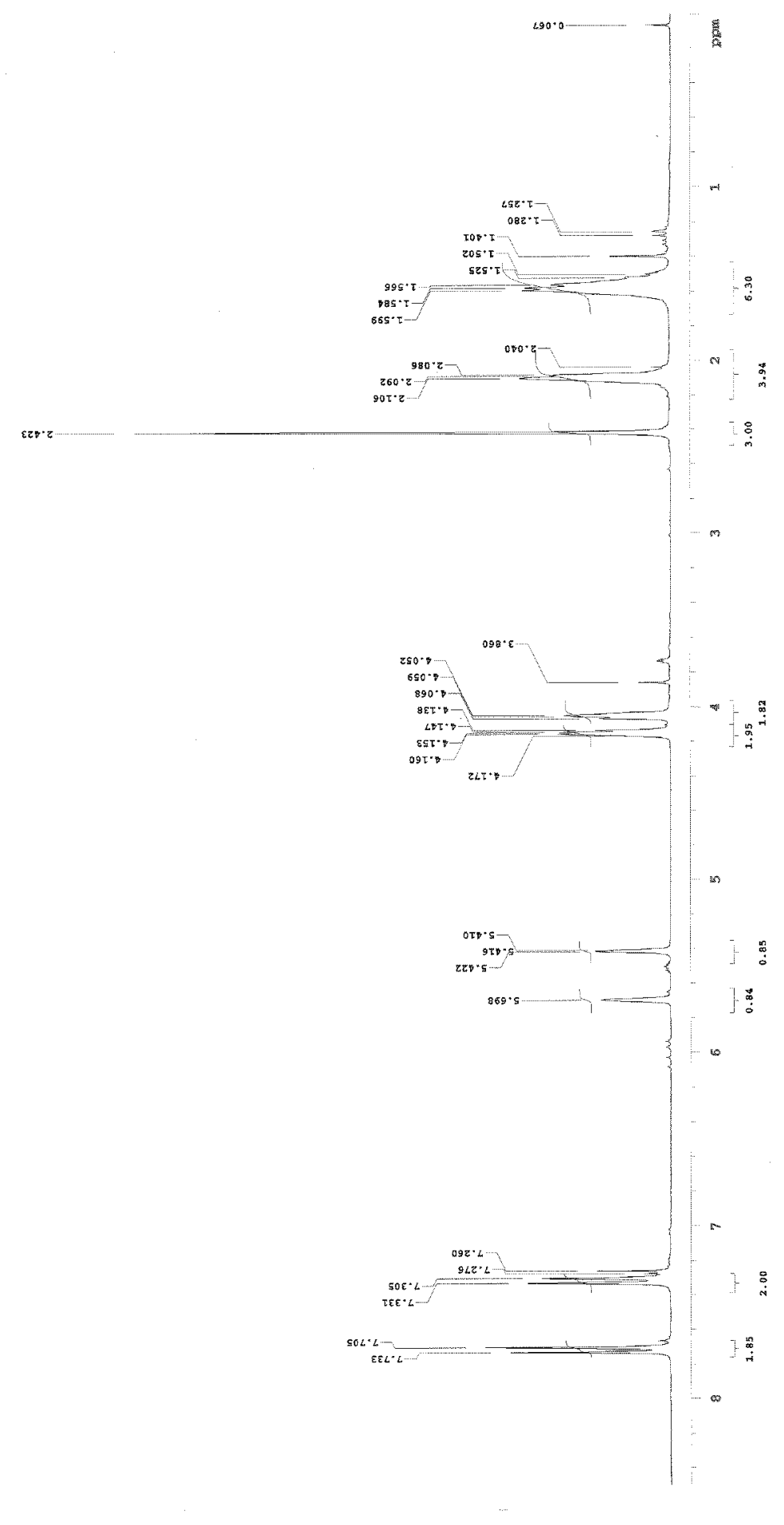




$$
\text { A }
$$




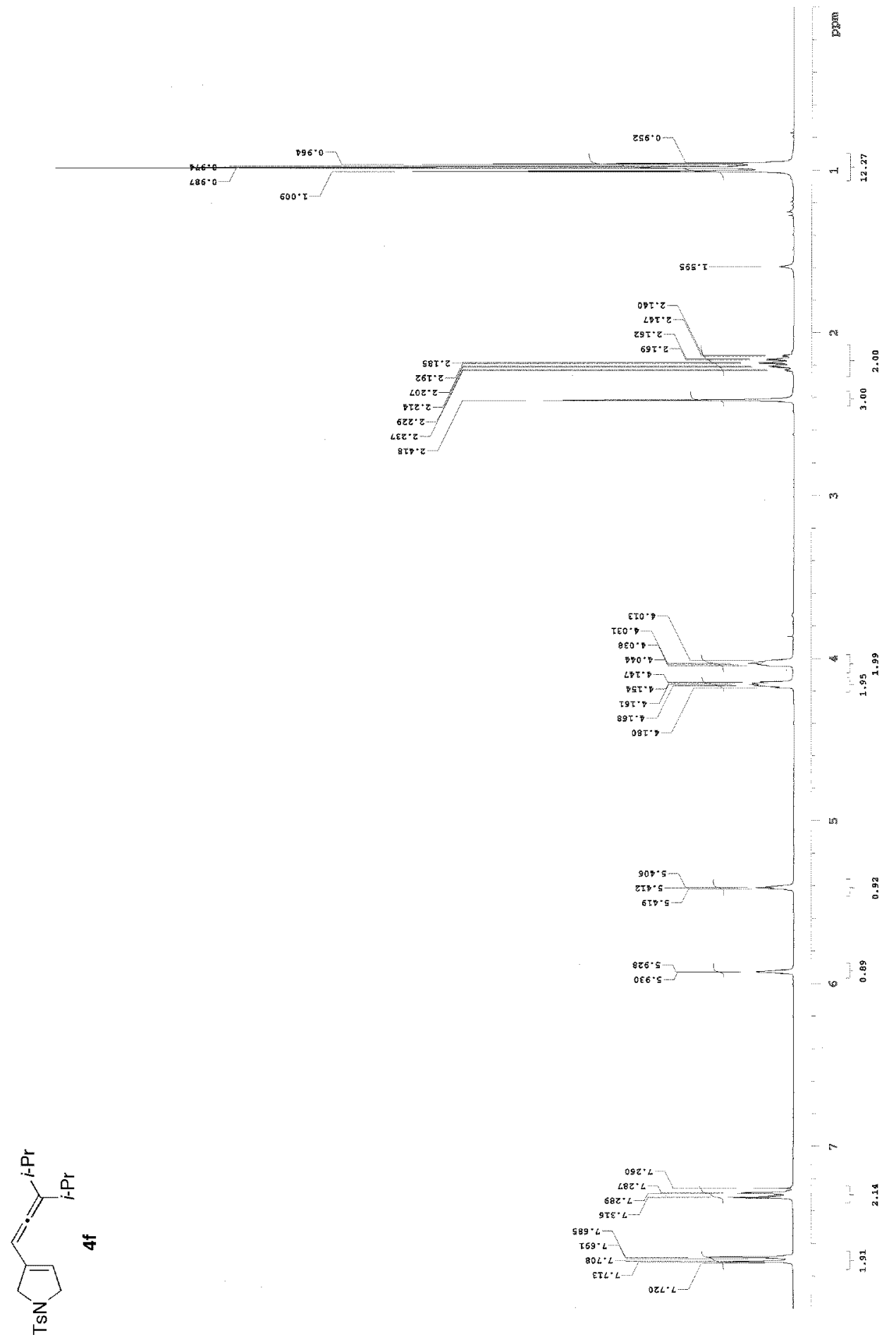




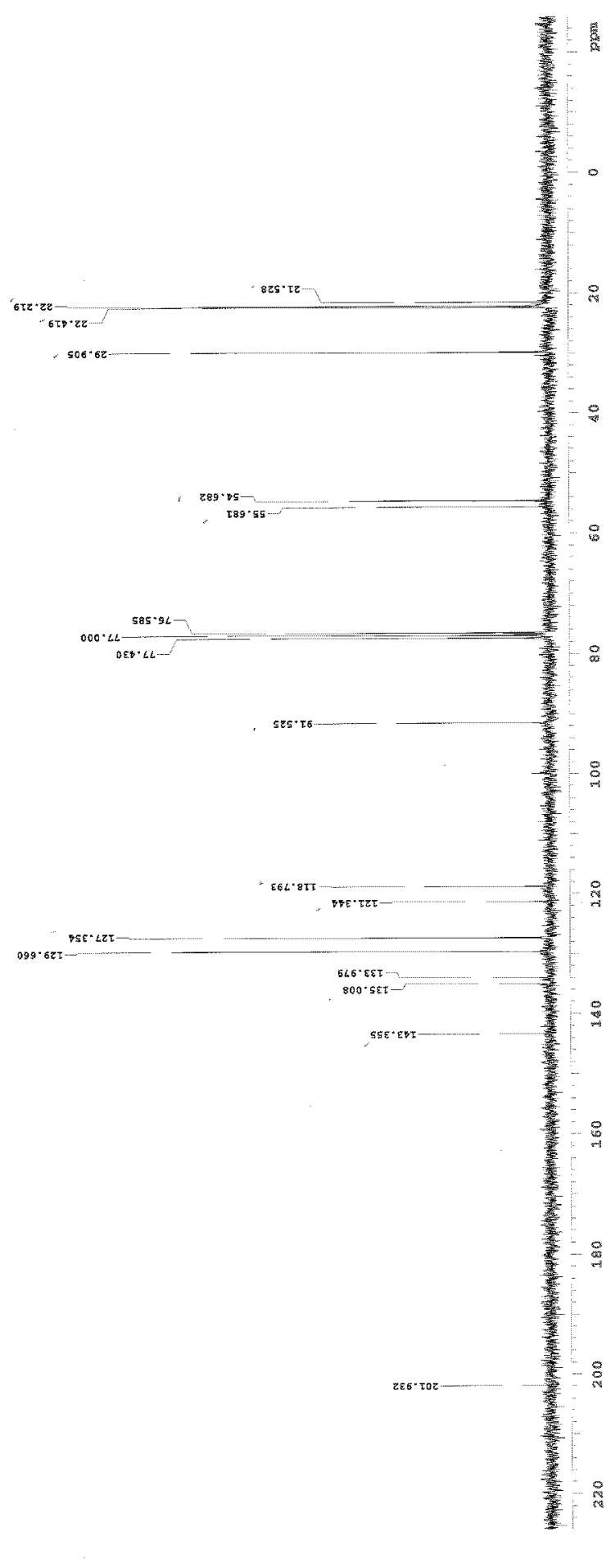




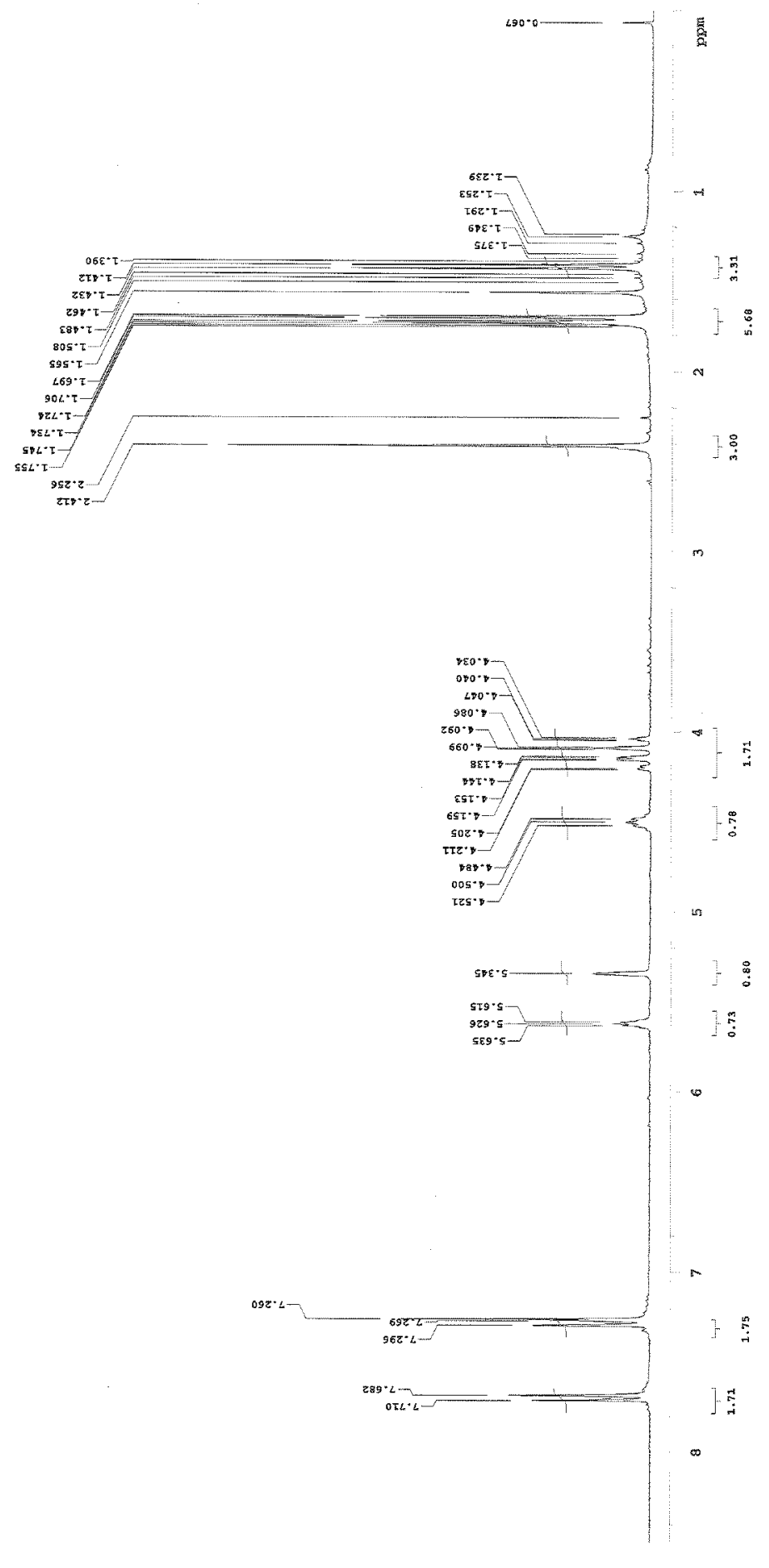




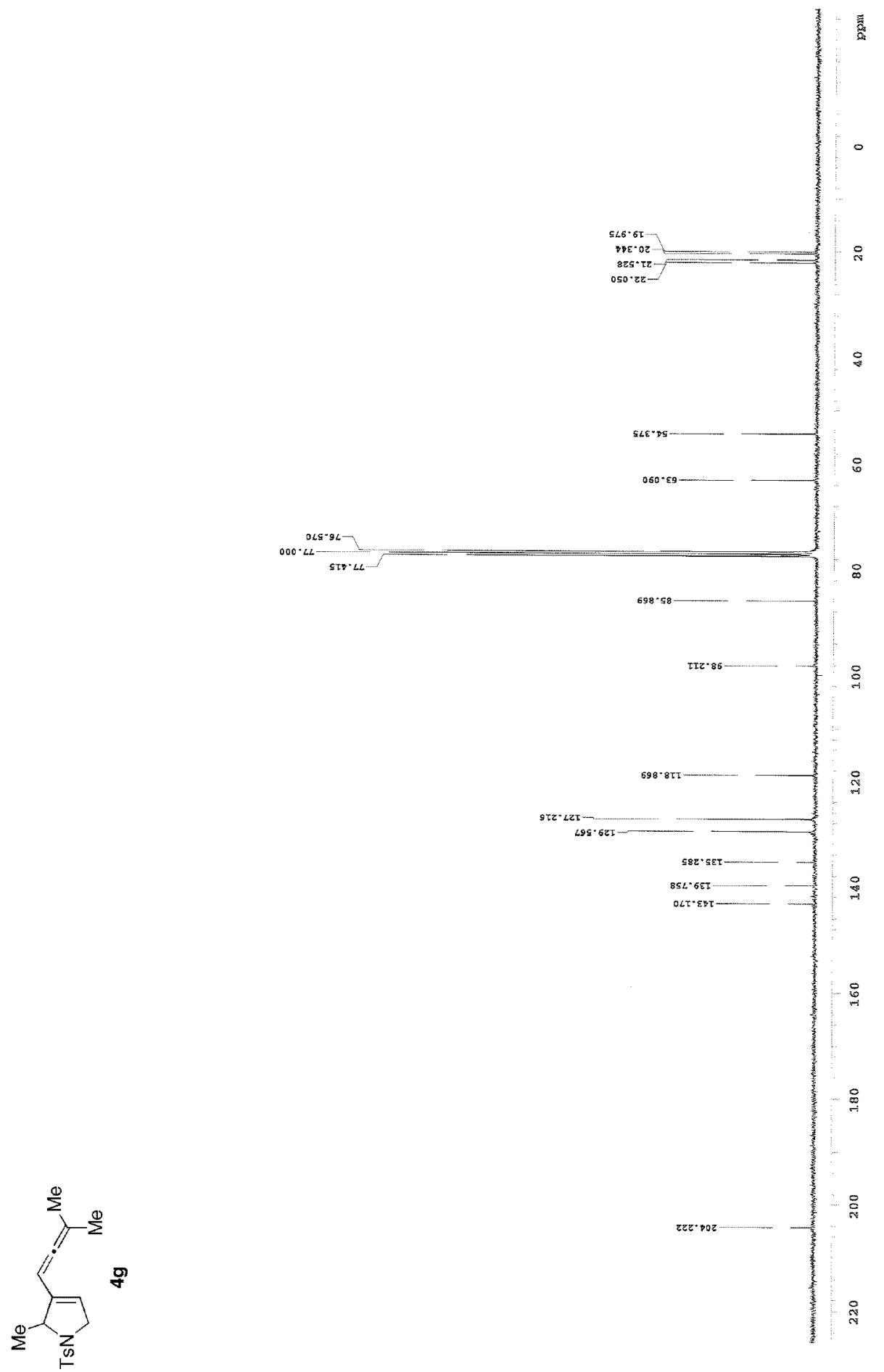




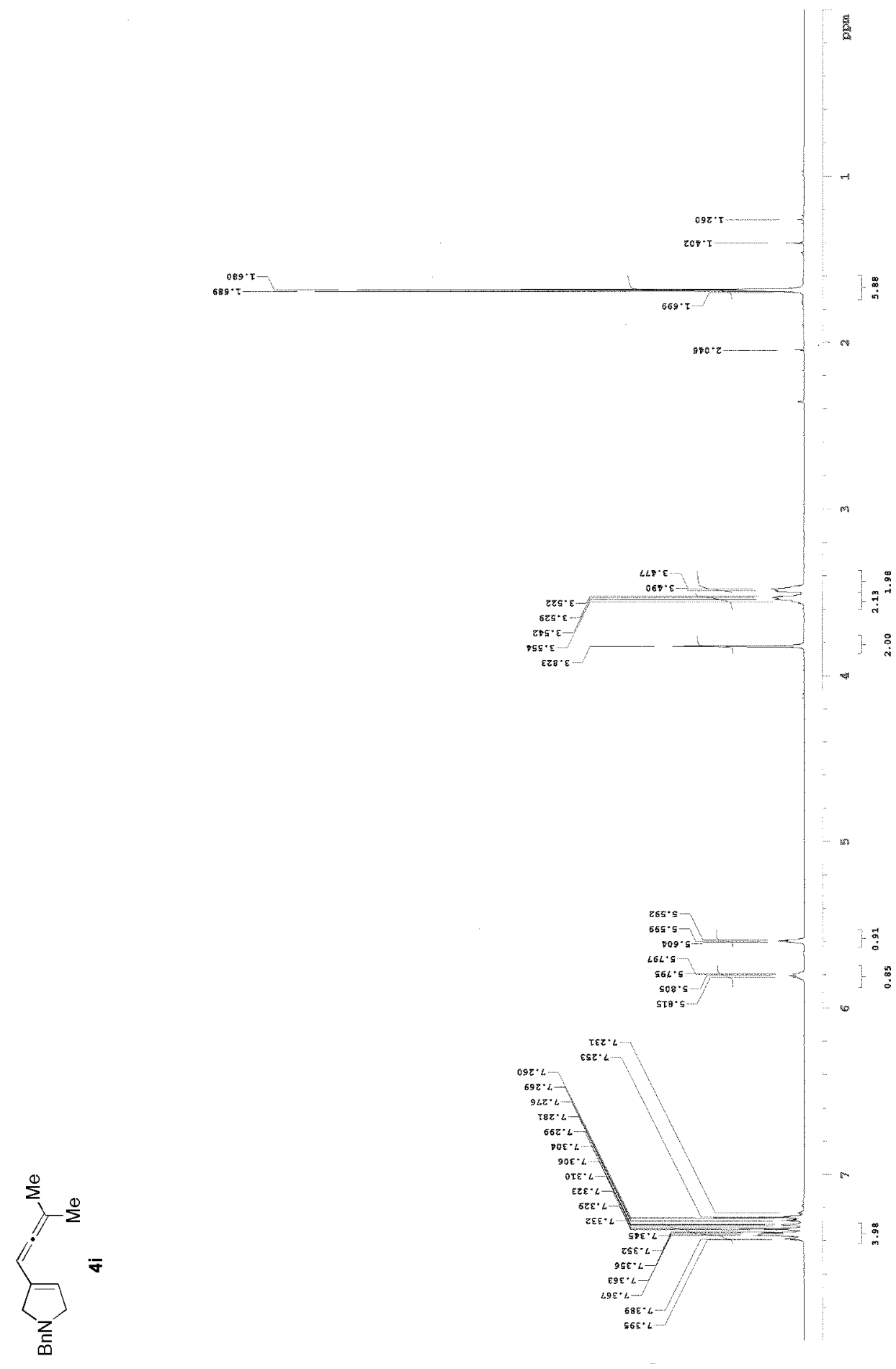




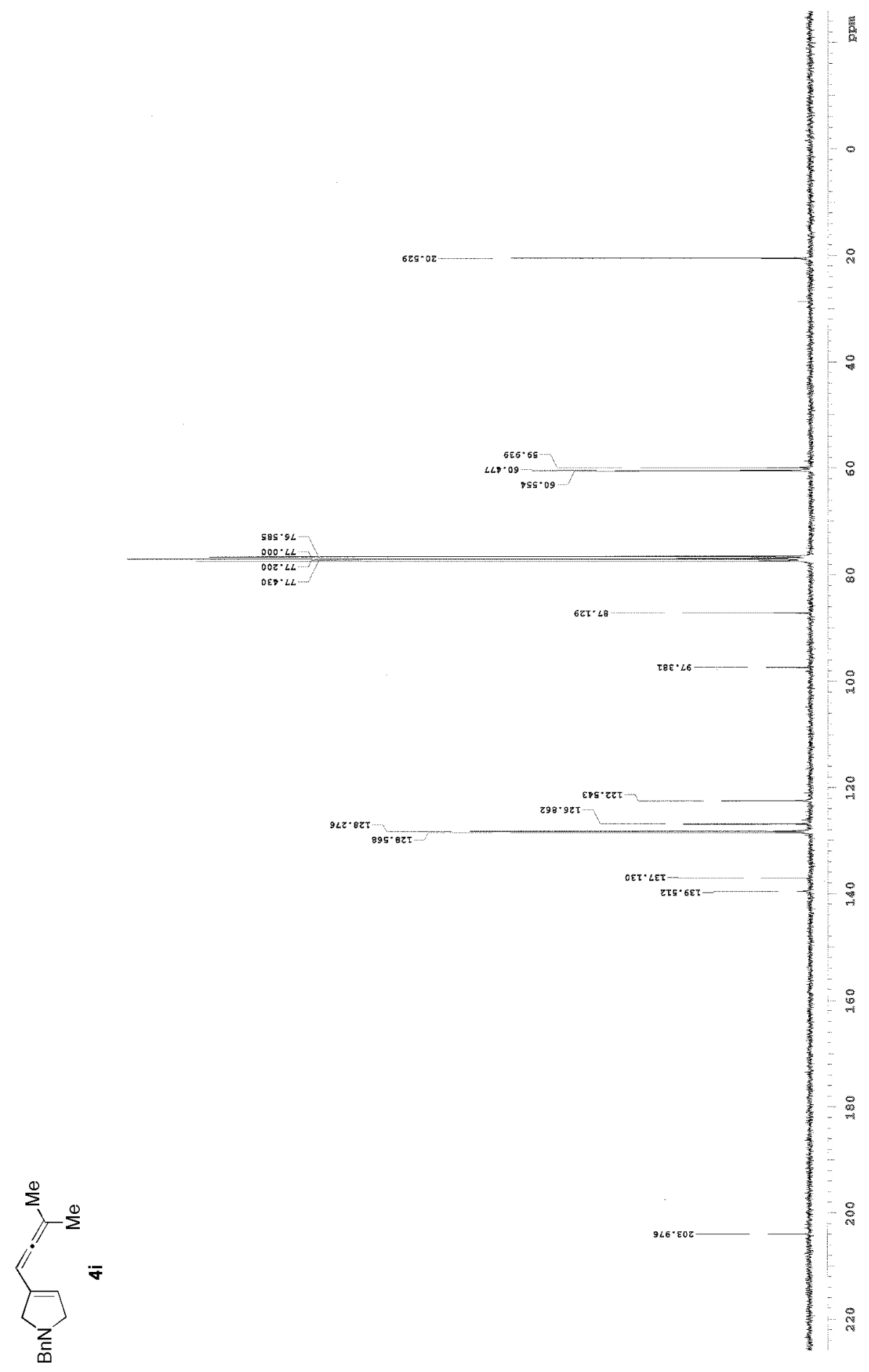




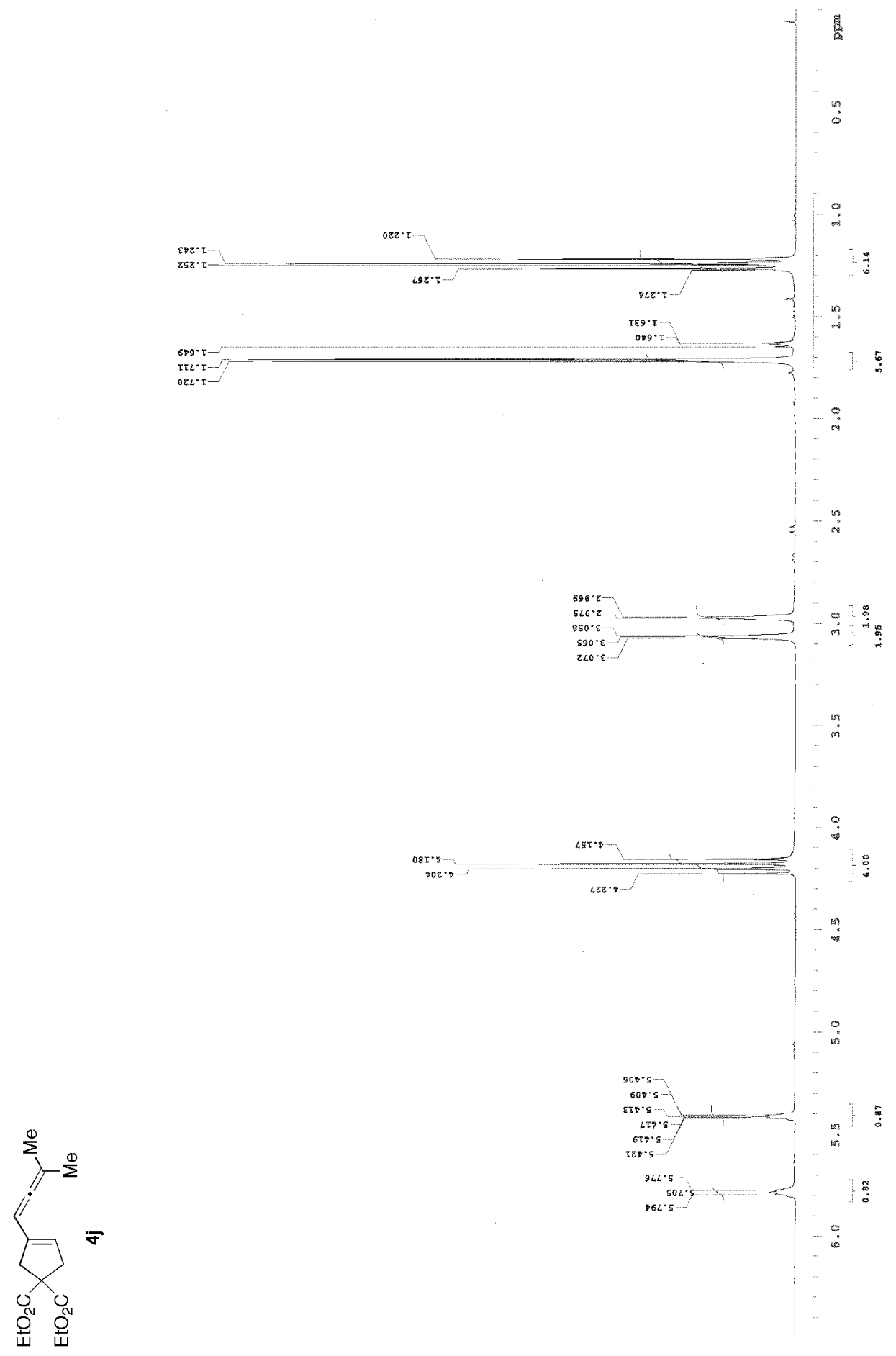




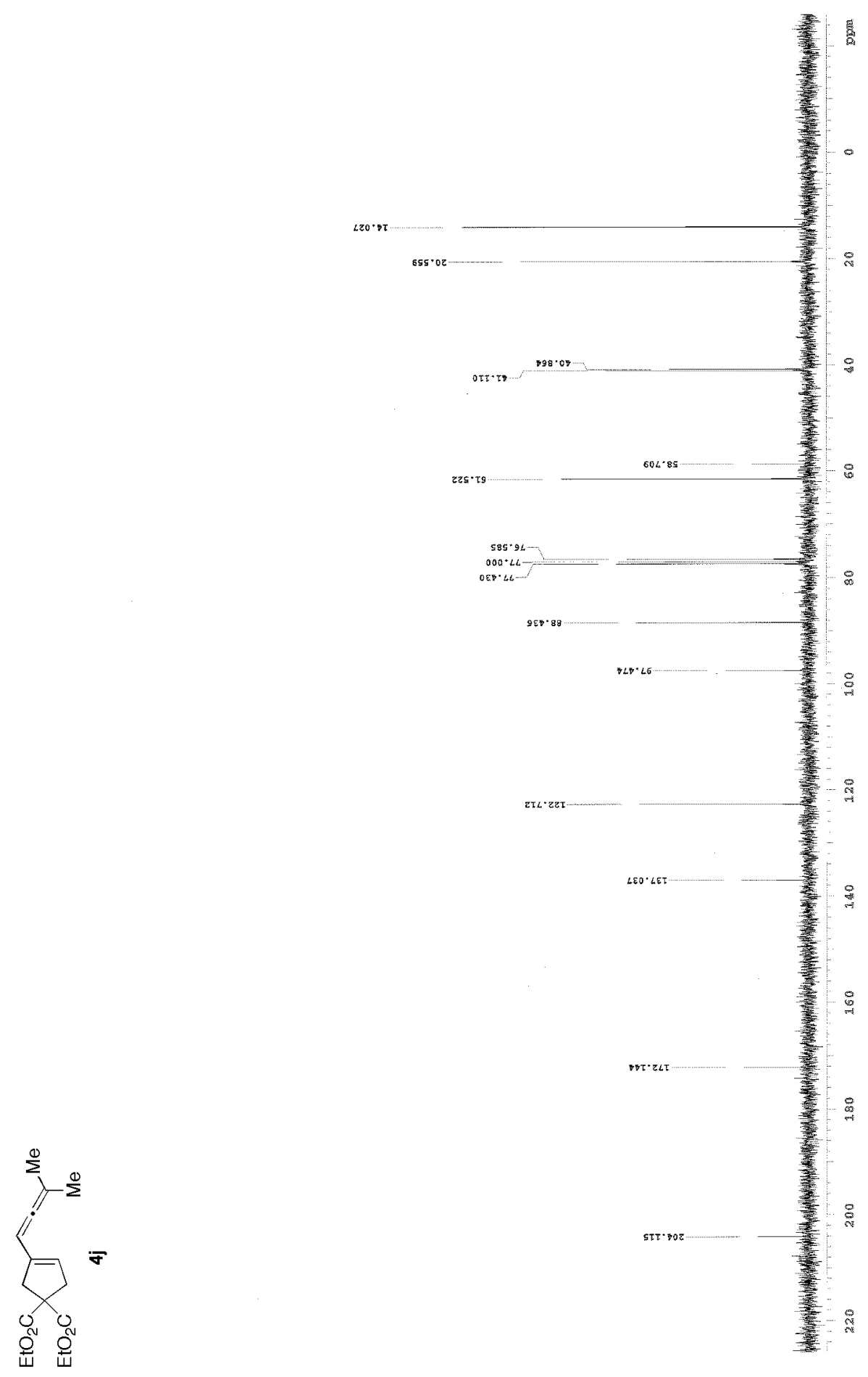




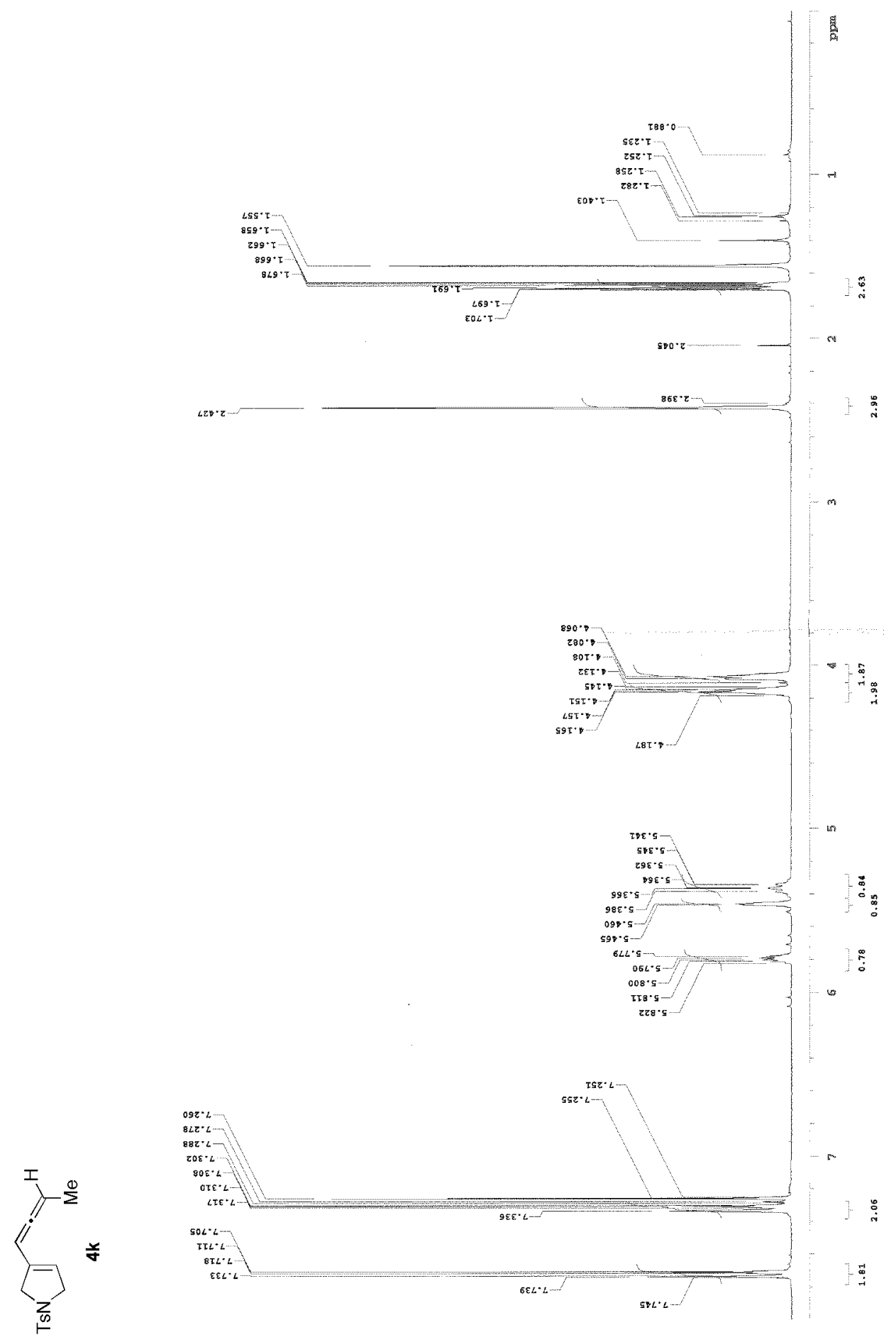




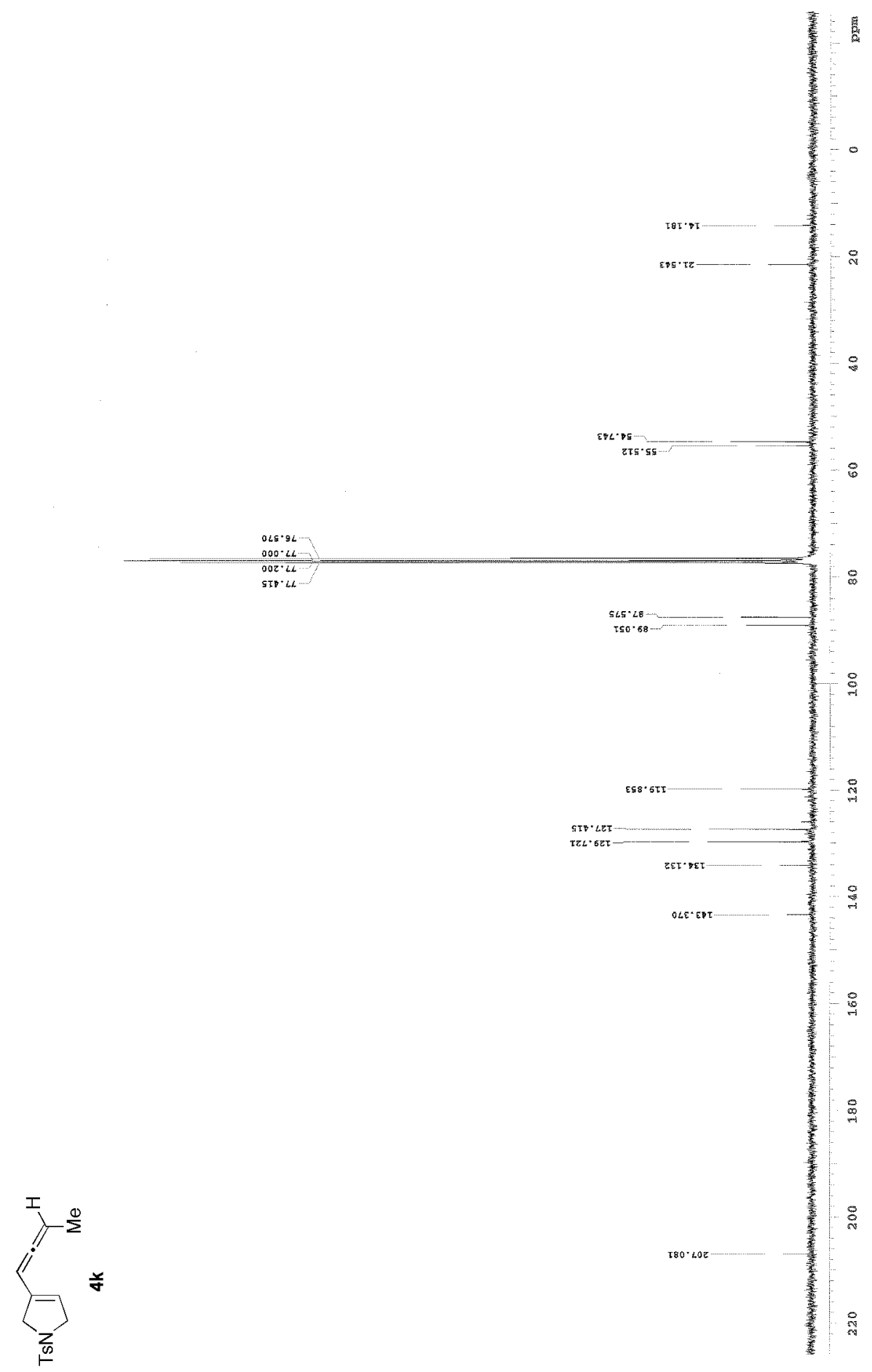



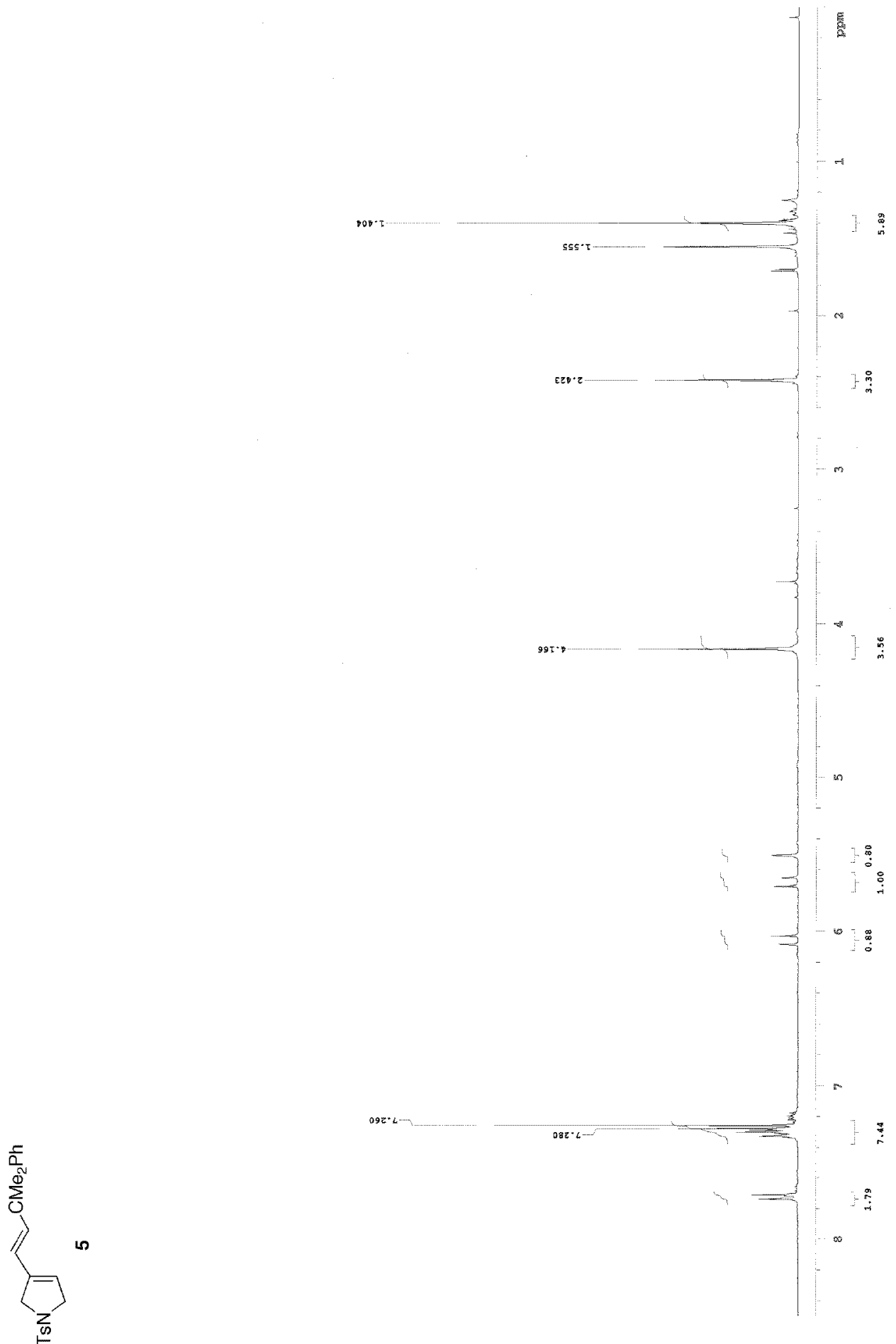


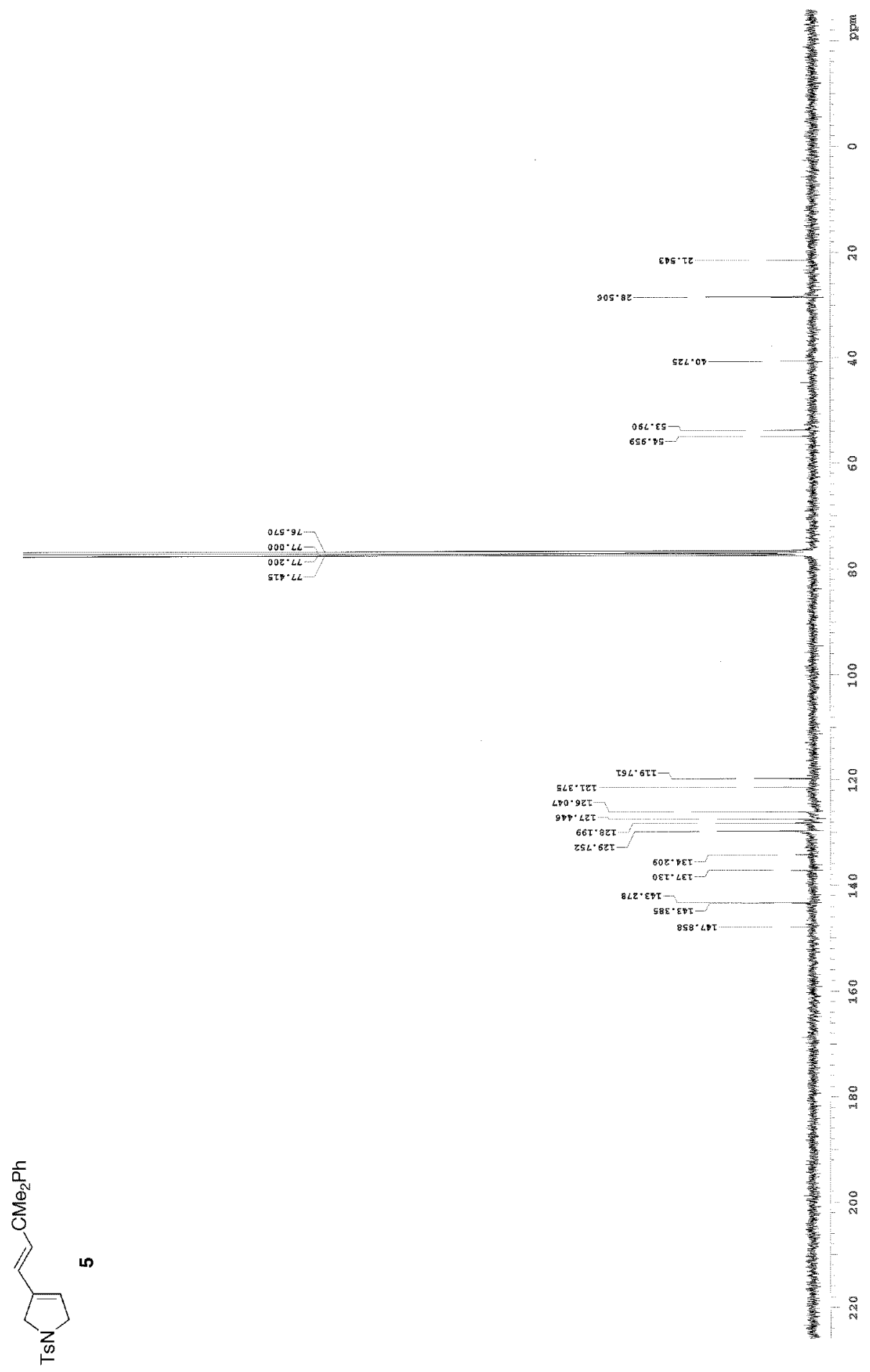




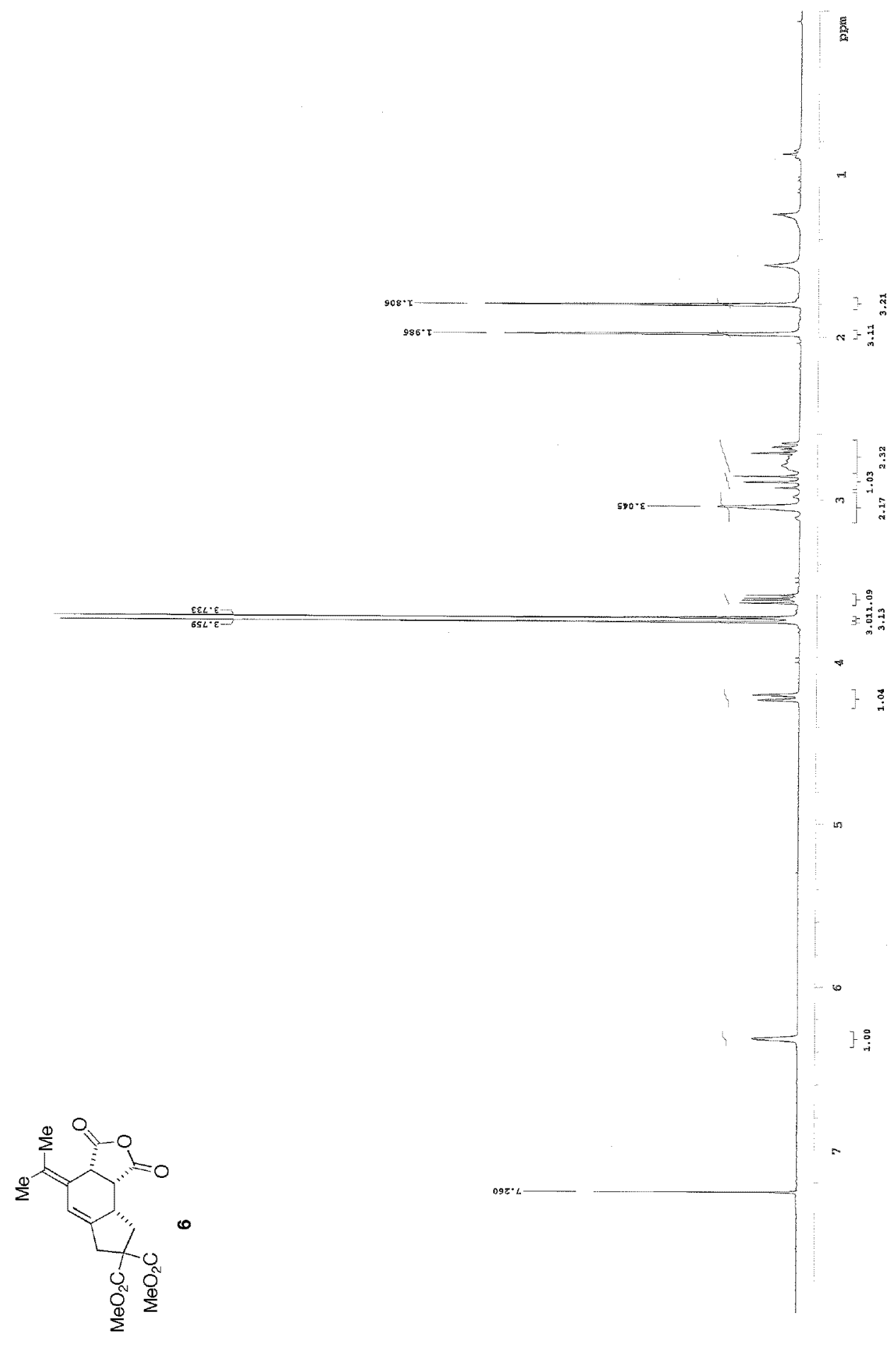




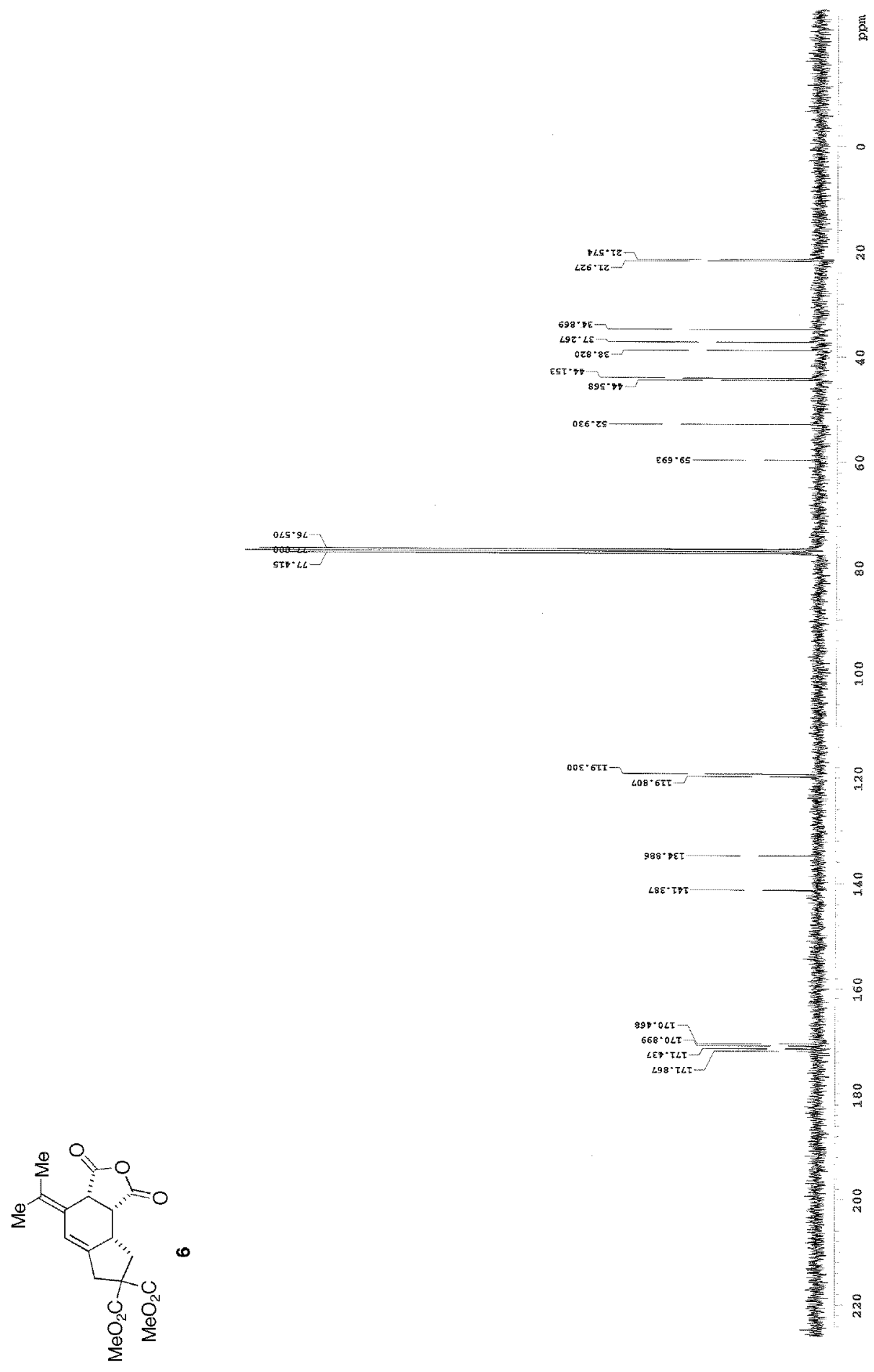

